\title{
Die Bruchlochwicklungen (Teillochwicklungen) und ihr Entwurf.
}

\author{
Von \\ Rudolf Richter.
}

Inhaltsübersicht.

Seite

I. Begriff der Bruchlochwicklung .. . . . . . . . . . . . . . . . 2I4

2. Der Spannungsstern . . . . . . . . . . . . . . . . . . . 2I6

A. Bruchlochwicklungen, bei denen alle Nuten bewickelt sind,

3. Die Symmetriebedingungen und die Ausführbarkeit . . . . . . . . . . . . . 220

4. Symmetrischc Bruchlochwicklungen, deren Nutenzahl $(N)$ und Polpaarzahl (p) keinen gemeinsamen Teiler haben $(\mathrm{t}=\mathrm{I})$. . . . . . . . . . . . . . . . . . . . 229

5. Symmetrische dreiphasige Bruchlochwicklungen, deren Nutenzahl $(\mathrm{N})$ und Polpaarzahl (p) als gröften gemeinsamen Teiler 2 haben $(t=2)$, und deren Spulenzahl im

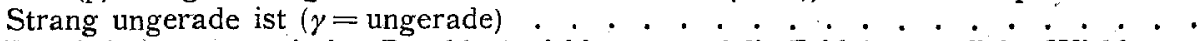

6. Die übrigen symmetrischen Bruchlochwicklungen und die Zahl der parallelen Wicklungszweige . . . . . . . . . . . . . . . . . . . . . . . . . . $24 \mathrm{I}$

B. Bruchlochwicklungen mit mehreren unbewickelten Nuten.

7. Die Ausführbarkeit . . . . . . . . . . . . . . . . . . . . . . . . . 246

8. Einphasige Bruchlochwicklungen . . . . . . . . . . . . . . . . . . . . 249

9. Symmetrische dreiphasige Bruchlochwicklungen mit nur drei unbewickelten Nuten $(\eta=3)$; Nutenzahl $(\mathrm{N})$, und Polpaarzahl $(\mathrm{p})$ teilerfremd $(\mathrm{t}=\mathrm{I})$. . . . . . . . 253

10. Die übrigen symmetrischen dreiphasigen Burchlochwicklungen mit nur drei unbewickelten Nuten $(\eta=3)$. . . . . . . . . . . . . . . . . . . . . . 256

II. Symmetrische dreiphasige Bruchlochwicklungen mit mehr als drei unbewickelten

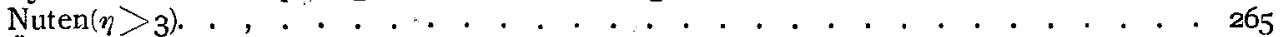

I2. Übersicht der behandelten Bruchlochwicklungen . . . . . . . . . . . 266

I. Begriff der Bruchlochwicklung. In den meisten Fällen werden die Wechselstromwicklungen so ausgeführt, daß sowohl das Verhältnis von gesamter Nutenzahl (Lochzahl) $\mathrm{N}$ und Produkt aus Polzahl 2p und Strangzahl ${ }^{1}$ ) $\mathrm{m}$

$$
\mathrm{Q}=\frac{\mathrm{N}}{2 \mathrm{pm}}=\operatorname{ganz}
$$

als auch das Verhältnis der Zahl der bewickelten Nuten $(\mathrm{N}-\eta)$ zu jenem Produkt,

$$
\mathrm{q}=\frac{\mathrm{N}-\eta}{2 \mathrm{pm}}=\text { ganz }
$$

ganze Zahlen sind. Man bezeichnet das Verhältnis $q$ als „Nutenzahl für Pol und Strang" und spricht, je nach der Größe von q, von I-, 2-, 3- oder q-Lochwicklungen.

Diese Wicklungen werden fast immer als Einschicht-Mantelwicklungen ausgeführt, q kann dann auch als das Verhältnis von Spulenzahl $\not$ eines Wicklungsstrangs und Polpaarzaht p,

gedeutet werden.

$$
\mathrm{q}=\frac{\mathrm{N}-\eta}{2 \mathrm{pm}}=\frac{\gamma}{\mathrm{p}}
$$

1) Gewöhnlich bezeichnet man immer noch die $m$ Wicklungsabteilungen einer Wicklung für m-Phasenstrom als die $m$ Phasen der Wicklung. Auf die Unzweckmäßigkeit dieser Bezeichnung hat zuerst G ö rge s (Elektrot. Zeitschrift 1895, S. 75I, Anmerkung in der dritten Spalte), später auch der Verfasser (Archiv f. Mathematik I904, S. 29I) hingewiesen. Der Vorschlag, die Wicklungsabteilungen als $Z$ weige zu bezeichnen, konnte sich nicht Geltung verschaffen, da jede Wicklungsabteilung im allgemeinen schon aus mehreren Wicklungszweigen besteht, die parallel oder in Reihe geschaitet werden können. Seit einigen Jahren bezeichne ich deshalb die den einzelnen Phasen des Mehrphasenstromes 'entsprechenden Abteilungen einer Wicklung als „Wicklungsstränge“, und kann deshalb die Bezeichnung "Wicklungszweige" für die Unterabteilungen in den Wicklungssträngen verwenden. Gleichwertige Wicklungszweige können parallel geschaltet werden; die Wicklungsstränge können in Stern oder in Vieleck geschaltet werden. 
Bei den Mehrphasenwicklungen sind gewöhnlich alle Nuten bewickelt,

$$
\eta=\mathrm{o}
$$

deshalb ist in diesem Falle immer

$$
q=Q
$$

Bei den Einphasenwicklungen bewickelt man gewöhnlich nur etwa ${ }^{2} / 3$ jeder Polteilung; es ist dann die Zahl der unbewickelten Nuten

$$
\eta=\gamma
$$

und es ist

$$
\mathrm{q}<\mathrm{Q} \text {. }
$$

Die Wicklungen, die den Bedingungen $\mathrm{I}$ a und $\mathrm{I} b$ genügen, haben die Eigenschaft, daß immer $\mathrm{p}$ Spulen desselben Wicklungsstrangs relativ zu den Polpaaren genau dieselbe Lage haben. Es lassen sich deshalb immer mindestens p gleichwertige Wicklungszweige herstellen, die in jedem Augenblick genau dieselbe Spannung haben und nach Bedarf parallel geschaltet werden können. Man könnte diese Wicklungen (Gl. I a und I b) als polpaarige Wicklungen bezeichnen, weil sie sich in gleichwertige Wicklungszweige zerlegen lassen, deren Zahl immer mindestens gleich der Zahl der Polpaare ist. Es ist jedoch unzweckmäßig, den Gattungsbegriff durch ein Beiwort (polpaarig) zu kennzeichnen, deshalb wollen wir die Wicklungen, bei denen sowohl $Q$ (Gl. I a) als auch q (Gl. Ib) ganz sind, als „Ganzlochwick l ung en" bezeichnen.

Wenn die Verhältnisse $Q$ und $q$ nicht gleichzeitig ganze Zahlen sind, wenn also entweder

$$
\mathrm{Q}=\frac{\mathbf{N}}{\mathbf{2 p m}}=\mathbf{g}+\frac{\mathbf{z}}{\mathbf{n}} \operatorname{oder} \mathbf{q}=\frac{\mathbf{N}-\eta}{\mathbf{2 p m}}=\mathbf{g}+\frac{\mathbf{z}}{\mathbf{n}}
$$

oder auch $Q$ und q Brüche mit dem Zähler $n g+z$ und dem Nenner $n$ sind $(z<n)$, so haben nicht mehr je $\mathrm{p}$ Spulen desselben Wicklungsstrangs relativ zu den Polpaaren genau dieselbe Lage; die Wicklung ist dann nicht mehr polpaarig. Solche Wicklungen wurden bisher mit dem Gattungsbegriff „Teillochwicklungen" bezeichnet, der aber an das Wesen der Wicklungen, die nicht den Bedingungen I a und $\mathrm{Ib}$ genügen, in keiner Weise erinnert. Wir wollen ihn deshalb hier durch „Bruchlochwicklungen“" ersetzen und damit zum Ausdruck bringen, daß min-. destens eines der Verhältnisse $Q$ und $q$ ein Bruch ist (Gl. 5).

Die Bruchlochwicklungen haben gegenüber den Ganzlochwicklungen den Vorteil, daß die Spulengruppen desselben Wicklungsstrangs unter sich in der Phase verschoben sind. Dadurch werden im allgemeinen die Wicklungsfaktoren der Oberwellen in höherem Maße verkleinert als der Wicklungsfaktor der Grundwelle, so daß die Spannungskurve sich mehr der Sinusform nähert als bei der Ganzlochwicklung mit ungefähr derselben Nutenzahl. Da bei den Bruchlochwicklungen die unter verschiedenen Polen liegenden Spulenseiten (Nuten) Phasenunterschiede aufweisen, die nur Bruchteile von dem Phasenunterschied benachbarter Nuten betragen, so müssen auch die Oberwellen der E.M.K., die von der Nutung des Ankers herrühren, mehr oder weniger vollkommen unterdrückt werden.

Zuweilen wird man auch zur Bruchlochwicklung geführt, wenn ein vorhandener Blechschnitt Verwendung finden soll, dessen Nutenzahl nicht durch die verlangte Pol- und Strangzahl teilbar ist, oder wenn mit Rücksicht auf die gute Ausnützung des Nutenraums (Hochspannungsmaschinen) die Zahl der Nuten so klein gewählt werden muß, daß eine Ganzlochwicklung überhaupt unmöglich $(\mathrm{q}<\mathrm{I})$ oder doch eine schlechtere Fórm der Spannungskurve ergibt.

Trotz dieser.Vorteile haben die Bruchlochwicklungen bisher nur wenig Eingang in die Praxis gefunden. Dies läßt sich vielleicht dadurch erklären, daß die Verfasser früherer Arbeiten über Bruchlochwicklungen ${ }^{1}$ ) nur gewisse Ausführungsbeispiele näher

1) Punga, Elektrot. Zeitschrift 1908, S.Ir8. S eidn er, Elektrot. u. Maschinenbau 19ro, S. 785. 
untersucht haben, ohne die praktisch wichtigsten Wicklungen zusammenzustellen und nach ihren Eigenschaften zu ordnen. Es soll hier versucht werden, die Bruchlochwicklungen systematisch zu behandeln und allgemeine Gesichtspunkte über den Entwurf solcher Wicklungen anzugeben. Dabei werden wir uns auf die symmetrischen Wicklungen beschränken, und von diesen Wicklungen auch nur die näher untersuchen, die bei derselben Nuten- und Polpaarzahl die beste Ausnutzung, also den größten Wicklungsfaktor der Grundwelle ergeben.

2. Der Spannungsstern. Wenn - wir für eine Wechselstromwicklung die Grundwellen der einzelnen Spulenspannungen nach Größe und Phase in einem Polardiagramm darstellen, so erhalten wir den Spannungsstern der Wicklung. Bei den gewöhnlichen Ganzlochwicklungen mit Spulen gleicher Weite (Abb. Ia) haben die Spannungen der am Ankerumfang aufeinander folgenden Spulen im allgemeinen verschiedene Phase, sind aber gleich groß, wenn alle Spulen dieselbe Windungszahl haben, wie es gewöhnlich der Fall ist. Bei den Wicklungen mit ungleichen Spulen-

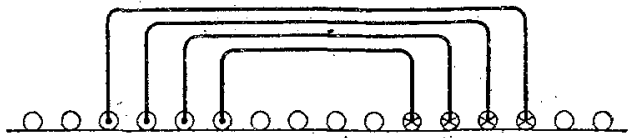

Abb. I b. Wicklungsköpfe von gleichachsigen Spulen uugleicher Weite.

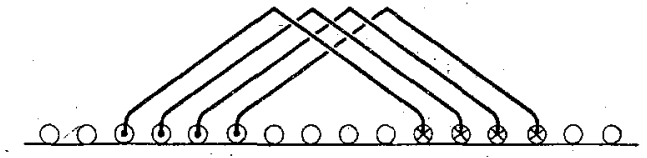

Abb. г a. Wicklungsköpfe von ungleichachsigen Spulen gleicher Weite.

weiten (Abb. I b) erhält man dagegen für die einzelnen Spulen im allgemeinen verschieden große Spannungen; die gleichachsigen Spulen haben jedoch dieselbe Spannungsphase. Es läßt sich leicht nachweisen, daß bei Reihenschaltung der Spulen die resultierende Spannung des Wicklungsstrangs unabhängig von der Reihenfolge ist, in der die einzelnen Spulenseiten zu Spulen miteinander verbunden werden, wenn nur der Sinn der einzelnen Spulenseiten, der in den Abb. I a und b durch Kreuze und Punkte angedeutet ist, unverändert bleibt. Zur Beurteilung der E.M.K., die in einer Spulengruppe oder in einem Wicklungsstrang induziert wird, genügt es deshalb, Sinn und Lage der Spulenseiten am Ankerumfang zu kennen.

Der Entwurf der Wicklung kann daher in zwei Teile zerlegt werden:

erstens Bestimmung der Lage der Spulenseiten, damit die verlangte resultierende E.M.K. nach Größe und Phase induziert wird,

zweitens Vereinigung der ungleichsinnigen Spulenseiten zu Spulen, so daß die technische Ausführung der Wicklung zweckentsprechend und möglichst einfach wird.

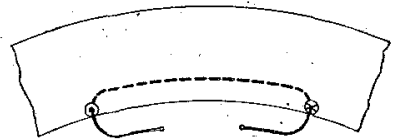

Abb. 2a. Mantelwindung.

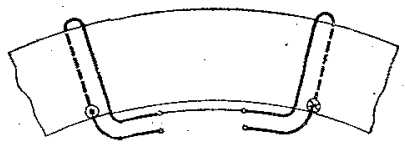

Abb ab. Vereinigung zweier Ringwindungen zur Mantelwindung.

Wir können uns nun jede Windung am Ankermantel (Abb. 2 a) durch zwei gegeneinander geschaltete Ringwindungen (Abb. 2 b), die um den Ankerkern geschlungen sind, ersetzt denken. Dadurch zerlegen wir die in einer Mantelwindung induzierte E.M.K. in zwei Teile, und können in diesem Sinne auch von dem Beitrag eines Ankerleiters oder auch einer Spulenseite zur gesamten E.M.K., oder kurz von der E.M.K. einer Spulenseite sprechen. Diese Teil-E.M.Ke. haben aus Symmetriegründen bei gleicher Leiterzahl in der Nut immer gleiche Größe, aber im allgemeinen verschiedene Phase, die sich aus der Lage der Nuten am Ankerumfang ergibt, in denen sich die Spulenseiten befinden.

Jeder Nut entspricht also ein Spannungsstrahl im Polatdiagramm.

Mit Rücksicht auf eịnfache und billige Herstellung der Maschinen werden die Nuten immer gleichmäßig über den ganzen Ankerumfang verteilt. Der Phasen- 
winkel benachbarter Nuten ist dann

$$
\alpha=\frac{\mathrm{p}}{\mathrm{N}} 360^{\circ}
$$

Bezeichnen wir mit $\mathrm{t}$ den, größten Teiler, den die Nutenzahl $\mathrm{N}$ und die Polparzahl $\mathrm{p}$ gemeinsam haben, so ergeben sich immer $t$ phasengleiche Nuten; denn es ist dann

$$
\frac{\mathrm{N}}{\mathrm{t}} \alpha=\frac{\mathrm{p}}{\mathrm{t}} 360^{\circ}=\mathrm{g} 360^{\circ}
$$

ein ganzes Vielfaches von $360^{\circ}$. Im Spannungsstern erhalten wir $\frac{\mathrm{N}}{\mathrm{t}}$ ungleichphasige Strahlen (Nuten), die um den Phasenwinkel

$$
a^{\prime}=\frac{t}{N} 360^{\circ}
$$

gegeneinander verschoben sind.

Für eine Ganzlochwicklung ist die Nutenzahl immer durch die Polpaarzahl teilbar, es ist also $t \doteq p$, so daß immer $\mathrm{p}$ Nuten phasengleich sind und $\boldsymbol{\alpha}^{\prime}=\boldsymbol{\alpha}$ ist. Wir wollen für eine vierpolige Dreiphasenwicklung mit 36 Nuten den Entwurf des Spannungssterns erläutern. Für diese Wicklung wird

$$
\mathrm{Q}=\mathrm{q}=\frac{36}{2 \cdot 2 \cdot 3}=3 \text {. }
$$

Numerieren wir die Nuten so, wie sie am Ankerumfang aufeinander folgen, so wird die Phase der Nuten oder die Größe und Phase der Spannungen der Spulenseiten durch die Strahlen in Abb. 3 a dargestellt, wobei die gleichphasigen Strahlen, z. B. der Nuten I und I9 der Deutlichkeit wegen aneinander gereiht sind.

Wir haben jetzt die Nuten auf die drei Wicklungsstränge zu verteilen. Jeder Strang erhält ${ }^{36}{ }_{3}=12$ Spulenseiten, je 6 positive und 6 negative, wie wir zur Abkürzung den Sinn bezeichnen wollen, in dem die entsprechenden Ringspulen zu schalten sind, damit Mantelspulen entstehen (vgl. Abb. $2 \mathrm{a}$ und b). Die gleichsinnigen Spulenseiten desselben Strangs sollen mit Rücksicht auf gute Ausnützung der Wicklung möglichst wenig Phasenunterschied aufweisen. Wir werden deshalb die positiven Spulenseiten des einen Strangs beispielsweise in den Nuten I, 2, 3, I9, 20 und 2 I unterbringen. Die entsprechenden Spannungsstrahlen sind

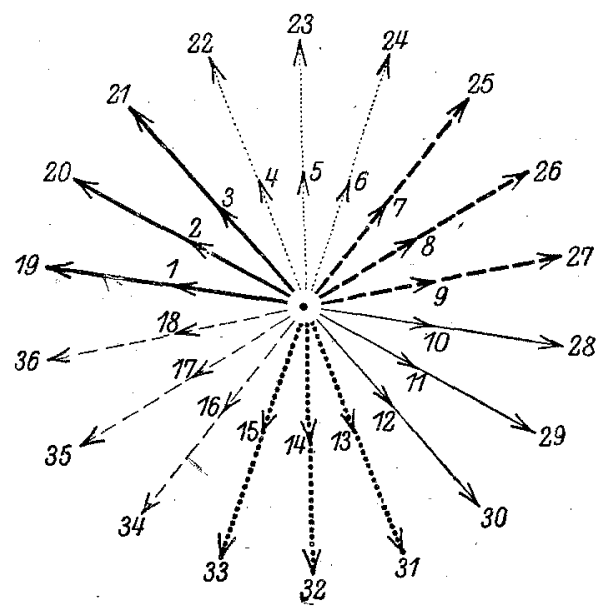

Abb. 3a. . Nutenstern einer dreiphasigen Ganzlochwicklung mit $N=36, p=2, \gamma=6$, $q=3$. durch dicke voll ausgezogene Linien gekennzeichnet. Die positiven Spulenseiten der andern Stränge müssen in Nuten liegen, die um je $\mathrm{I}_{20}{ }^{\circ}$ in der Phase verschoben sind, damit eine symmetrische Wicklung entsteht. Wir erhalten hierbei die Nuten, deren Strahlen durch dicke gestrichelte und punktierte Linien angedeutet sind. Die negativen Spulenseiten jedes Strangs müssen wieder mit Rücksicht auf gute Aüsnützung der Wicklung möglichst wenig Phasenunterschied aufweisen; aber gegenüber den entsprechenden positiven Spulenseiten um möglichst $180^{\circ}$ in der Phase verschoben sein. Die negativen Spulenseiten sind deshalb in die Nuten zu wickeln, deren Strahlen durch dünne Linien gekennzeichnet sind (vgl. Abb. 3 a).

Hiermit ist der Spannungsstern aufgezeichnet, aus dem man die Wicklung ableitet, indem je zwei beliebige aber ungleichsinnige Spulenseiten desselben Wick- 
lungsstrangs durch Spulenköpfe zu Spulen verbunden werden. Durch diese Vereinigung (Gegenschaltung) zweier Ringspulen zu Mantelspulen ändert sich der Sinn der dünn ausgezogenen Spannungsstrahlen, so daß die Teilspannungen des aus Mantelspulen bestehenden Wicklungsstrangs durch die Strahlen in Abb. $3 \mathrm{~b}$ (verkleinerter Maßstab) dargestellt werden. Zur Abkürzung wollen wir den Spannungsstern in Abb. 3 a als „Nutenstern" bezeichnen. Bei Reihenschaitung sämtlicher Spulen desselben

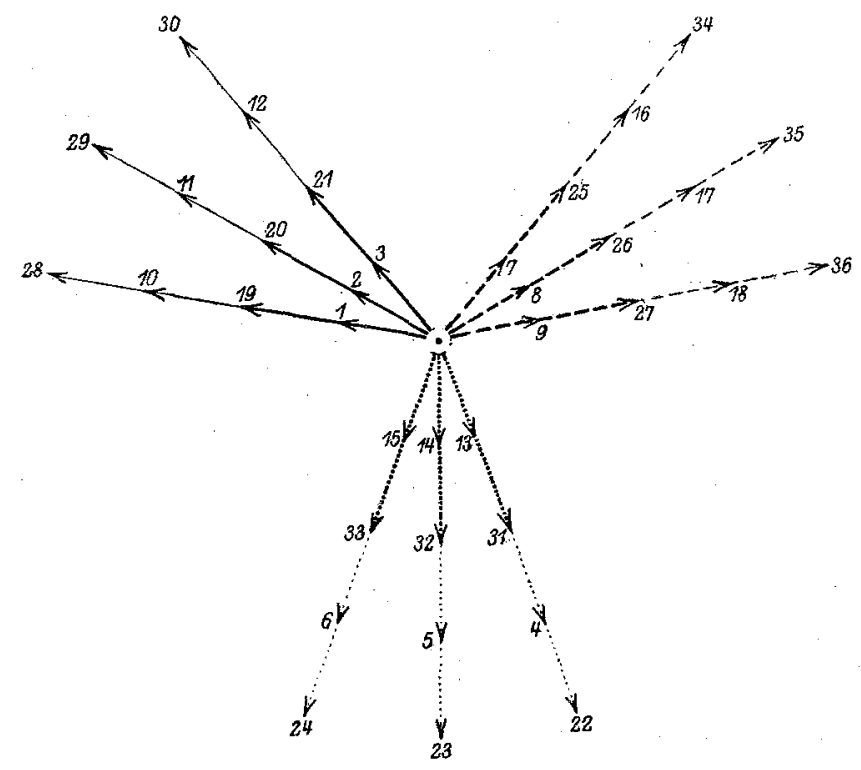

Abb. 3 b. Einzelspannungen für die Wicklung zu Abb. $3^{a}$.
Wicklungsstrangs sind die Teilspannungen geometrisch zu addieren, und wir erhalten (in kleinerem Maßstabe) die Spannungen der drei Wicklungsstränge in $\mathrm{Abb} .3 \mathrm{c}$.

$\mathrm{Da}$ bei den Ganzlochwicklungen die Spannungs sterne aller Polpaare übereinstimmen, hätte es natürlich ge-

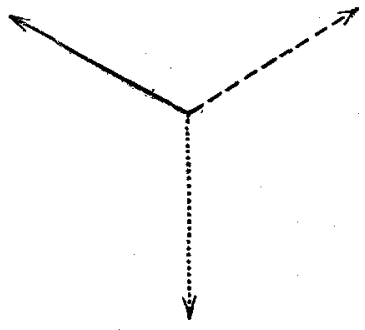

Abb. 3c. Resultierende Spannungen der Wicklungsstränge.

nügt, den Stern für ein einziges Polpaar zu zeichnen; wir haben hier alle Spannungsstrahlen aufgezeichnet, um das Grundsätzliche über den Entwurf des Spannungssterns (vgl. auch S. 23I) zu erläutern, der für den Entwurf der Wicklung erst praktische Bedeutung gewinnt, wenn $q$ eine gebrochene Zahl ist.

Wir können auch den Spannungssternen in Abb. 3 a und b entnehmen, wieviel Zweige in jedem. Wicklungsstrang parallel geschaltet werden dürfen. Die Spannungen dieser Zweige müssen nach Größe und Phase gleich sein. Es ist deshalb aus dem Stern der Einzelspannungen festzustellen, in wie viele Gruppen sich

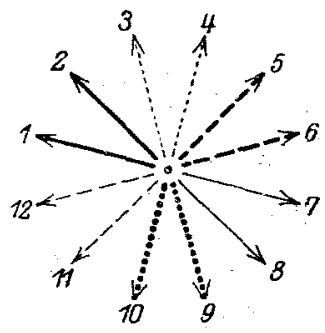

Abb. 4 a. Nutenstern einer dreiphasigen Ganzlochwicklung mit $N=12, p=I, \gamma=2, q=2$.

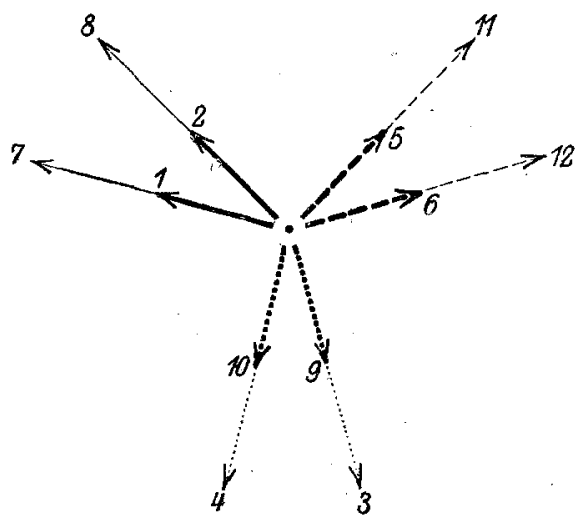

Abb. 4 b. Einzelspannungen für die Wicklung zu Abb. 4a.

die Strahlen desselben Strangs zerlegen lassen, so daß jede Gruppe gleich viele diclie und dünne Strahlen enthält und denselben resultierenden Strahl nach Größe und Phase ergibt. 
Bei den Ganzlochwicklungen sind immer p parallele Wicklungszweige möglich, weil sich die Phasen von $p$ Nuten im Spannungsstern stets decken, und für die Nuten jedes der p Spannungssterne eine Ganzlochwicklung ausführbar ist. Wenn die Nutenzahl q für Pol und Strang eine ge ra d e Zahl ist, lassen sich bei den Ganzlochwicklungen die Strahlen jedes der p Spannungssterne noch in je zwei Gruppen zerlegen, deren resultierende Strahlen gleiche Größe und Phase haben und ebenso vielen positiven wie negativen Spulenseiten entsprechen (vgl. Abb. 4b). Bei geradzahligem q sind deshalb immer $2 \mathrm{p}$ parallele Wicklungszweige möglich. In den Spannungssternen Abb. $4 \mathrm{a}$ und $\mathrm{b}$, die für eine Ganzlochwicklung mit $\mathrm{q}=2$ und $\mathrm{p}=\mathrm{I}$ gelten, ergeben z. B. die Spulenseiten der Nuten 2 und 7 , wenn sie zu Spulen verbunden werden, dieselbe Spannung nach Größe und Phase wie die Spulenseiten I und 8.

Die Ausnützung der Wicklung wird durch die Abweichung des Wicklungsfaktors der Grundwelle von der Einheit beurteilt. Der Wicklungsfaktor ist gleich dem Verhältnis von geometrischer Summe zu algebraischer Summe der zu demselben Wicklungsstrang gehörigen Teilspannungen, und läßt sich aus dem Spannungsstern leicht zeichnerisch oder rechnerisch bestimmen.

Wenn sich die resultierende Spannung ( $R$ ) eines

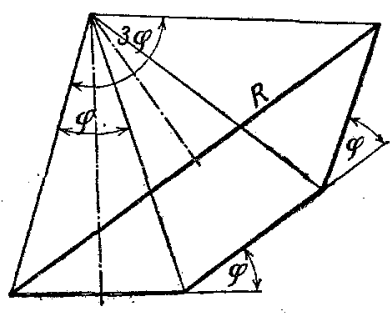

Abb. 5. Zur Berechnung des Wicklungsfaktors.

Wicklungsstranges aus s Teilspannungen gleicher Größe zusammensetzt, die je um den Phasenwinkel $\varphi$ gegeneinander verschoben sind (vgl. Abb. 5 mit s=3), so ist der Wicklungsfaktor

$$
\xi=\frac{\sin \frac{s \varphi}{2}}{s \sin \frac{\varphi}{2}} .
$$

Für eine Ganzlochwicklung ist $s=q$, der Phasenwinkel $\varphi$ ist nach Gl. 6 für die Grundwelle der Spannung

$$
\varphi=\alpha=\frac{\mathrm{p}}{\mathrm{N}} 360^{\circ}
$$

wofür wir nach Gl. I a und 3 auch schreiben können

$$
\varphi=\frac{\mathrm{I} 80^{\circ}}{\mathrm{m} \mathrm{q}} \text {. }
$$

Wenn alle Spulen dieselbe Windungszahl haben, erhalten wir also den Wicklungsfaktor der Grundwelle zu

$$
\xi_{1}=\frac{\sin \frac{90^{\circ}}{\mathrm{m}}}{\mathrm{q} \sin \frac{\mathrm{I}}{\mathrm{q}} \frac{90^{0}}{\mathrm{~m}}} .
$$

Die Wicklungsfaktoren der Oberwellen sollen ihrem absoluten Betrage nach im allgemeinen möglichst kleir sein, damit sich die Spannungskurve der Maschine möglichst der Sinusform nähert.

Für die $\imath \stackrel{\text { te }}{=}$ Spannungswelle einer Ganzlochwicklung ist der Phasenwinkel

und der Wicklungsfalktor

$$
\varphi=\nu \alpha
$$

$$
\xi_{v}=\frac{\sin v \frac{90^{\circ}}{\mathrm{m}}}{\mathrm{q} \sin \frac{\nu}{\mathrm{q}} \frac{90^{0}}{\mathrm{~m}}} .
$$


In Tafel 1 sind die Wicklungsfaktoren der dreiphasigen $(\mathrm{m}=3)$ Ganzlochwicklungen,

$$
\xi_{\nu}=\frac{\sin \nu 30^{\circ}}{\mathrm{q} \sin \frac{\nu 0^{\circ}}{\mathrm{q}}}
$$

für die Spannungswellen ungerader Ordnung bis $\boldsymbol{\nu}=$ I I und verschiedene Werte von q zusammengestellt. Dabei ist hier, wie auch bei den übrigen Tafeln der Wicklungsfaktoren, das Vorzeichen außer acht gelassen, weil es bei unseren Betrachtungen nicht auf die genaue Form der Spannungskurve, sondern nur auf die Abweichung von der Sinusform ankommt ${ }^{1}$ ).

Tafel I.

Wicklungsfaktoren der drejphasigen Ganzlochwicklungen sowie der dreiphasigen Bruchlochwicklungen im Abschnitt 4, wenn q durch $\gamma$ ersetzt wird.

\begin{tabular}{|c|c|c|c|c|c|c|}
\hline \multirow[b]{2}{*}{$\begin{array}{l}\mathrm{q} \\
-\end{array}$} & \multicolumn{6}{|c|}{$\boldsymbol{v}=$} \\
\hline & I & 3 & 5 & 7 & 9 & II \\
\hline$I$ & 1,000 & $\mathrm{I}, 000$ & $I, 000$ & 1,000 & 1,000 & $\mathrm{I}, 000$ \\
\hline 2 & 0,966 & $0,7^{\circ} 7$ & 0,259 & $0,25 \dot{9}$ & 0,707 & 0,966 \\
\hline 3 & 0,960 & 0,667 & 0,217 & 0,178 & 0,333 & 0,178 \\
\hline 4 & $0,95^{8}$ & 0,653 & 0,204 & $\mathrm{o}, \mathrm{I} 57$ & $0,27 \mathrm{I}$ & 0,126 \\
\hline 6 & $0,95^{6}$ & 0,642 & 0,197 & $0, \mathbf{r}_{45}$ & 0,236 & 0,102 \\
\hline Io & 0,955 & 0,639 & 0,193 & 0,139 & 0,220 & 0,092 \\
\hline 20 & 0,955 & 0,637 & o,19т & 0,137 & $0,2 I_{4}$ & 0,088 \\
\hline$\infty$ & 0,955 & 0,637 & o,I9I & 0,136 & 0,212 & 0,088 \\
\hline
\end{tabular}

\section{A. Bruchlochwicklungen, bei denen alle Nuten bewickelt sind.}

3. Die Symmetriebedingungen und die Ausführbarkeit. Man nennt im allgemeinen eine $m$-Phasenwicklung symmetrisch, wenn die Spannungen an den $\mathrm{m}$ Wicklungssträngen gleich groß und gegeneinander um den Phasenwinkel $\frac{\mathrm{I}}{\mathrm{m}} 360^{\circ}$ verschoben sind. Eine solche Wicklung läßt sich stets ausführen, wenn es möglich ist, je $\mathrm{m}$ Spulen zu wickeln, deren Spannungen denselben Symmetriebedingungen genügen, die an die resultierenden Spannungen der Wicklungsstränge gestellt werden. Jede Mantelspule entsteht nun durch Vereinigung von $z$ wei wirksamen Spulenseiten; deshalb müssen sich, damit eine symmetrische m-Phasenwicklung mit einer Spulenseite in jeder Nut ausführbar ist, die N Nuten oder Spulenseiten der Wicklung immer in eine gerade Anzahi Gruppen einteilen lassen, von denen jede Gruppe $m$. Spulenseiten enthält, die um den Phasenwinkel $\frac{\mathrm{I}}{\mathrm{m}} 360^{\circ}$ gegeneinander verschoben sind. 'Die Einzelspannungen von je zwei Gruppen müssen dabei auch gleiche Größen haben, damit immer je m Spulen gebildet werden können, deren

1) Es sei daran erinnert, daß die Wicklungsfaktoren für einen Wicklungsstrang gelten, und daß fär die äußeren Klemmen einer symmetrischen Dreiphasen-Wicklung die Spannungswellen, deren Ordnungszahl durch 3 teilbar ist, null, während die übrigen Wellen ungerader Ordnungszahl $\sqrt{3}$ mal so gro $\beta$ sind wie die entsprechenden Strangsspannungen. 
Spannungen gleich groß sind. Wenn diese Bedingungen erfüllt sind, lassen sich die Spulenseiten so schalten, daß die resultierenden Spannungen der Wicklungsstränge den Symmetriebedingungen genügen ${ }^{1}$ ).

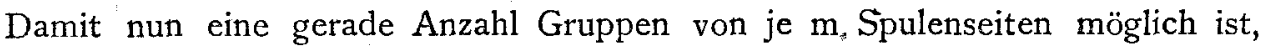
muß bei der üblichen Einschichtwicklung, wo jede Nut nur eine Spulenseite erhält,

$$
\frac{\mathrm{N}}{2 \mathrm{~m}}=\gamma
$$

sein. Darin ist $\gamma$ die Zahl der Spulen in jedem der Wicklungsstränge; $\gamma$ kann alle Zahlen des Zahlensystems durchlaufen.

Damit ferner die m Spannungen jeder Gruppe gegeneinander um den Winkel $\frac{\mathrm{I}}{\mathrm{m}} 360^{\circ}$ in der Phase verschoben sind, muß die Zahl der Strahlen ungleicher Phase durch die Strangzahl $\mathrm{m}$ teilber sein. Die Zahl der ungleichphasigen Strahlen ist nun nach Abschnitt 2 und Gl. I2a

$$
\frac{\mathrm{N}}{\mathrm{t}}=\frac{2 \mathrm{~m} \gamma}{\mathrm{t}}
$$

worin $t$ der größte Teiler ist, den die Nutenzahl $N$ und die Polpaarzahl p gemeinsam haben. Es muß also gelten:

$$
\frac{2 \gamma}{t}=\text { ganz }
$$

Außerdem müssen immer je $2 \mathrm{~m}$ Nuten dieselbe in Reihe geschaltete Leiterzahl erhalten,: damit die Spannungen von je m Spulen auch gleich groß werden. Bei der Aufzeichnung der Spannungssterne werden wir der Einfachheit wegen voraussetzen, daß in alle n Nuten gleich viele Leiter liegen.

Die Gl. $12 \mathrm{a}$ und b dürfen wir übrigens nicht $z u$ der Gleichung

$$
\frac{\mathrm{N}}{\mathrm{mt}}=\operatorname{ganz}
$$

zusammenfassen, weil dann die in Gl. I 2 a enthaltene Bedingung, daß $\mathrm{N}$ immer gerade sein soll, verschwinden würde.

Die in der Elektrotechnik unter dem Namen $Z$ weiphasenwicklung verwendete Wicklung ist in Wirklichkeit eine Vierphasenwicklung $(\mathrm{m}=4)$, bei welcher die um $180^{\circ}$ phasenverschobenen Wicklungsstränge, also der $\mathbf{I}$. und 3 . sowie der 2. und 4. Strang gegeneinander geschaltet sind, so daß aus den vier Wicklungssträngen zwei Wicklungsstränge entstehen. In Übereinstimmung mit dem Sprachgebrauch wollen wir diese. Zweiphasenwicklung symmetrisch nennen, wenn die

1) Wenn die Zahl der in Reihe geschalteten Windungen in allen Spulen dieselbe ist (gleiche Größze der Einzelstrahlen im Spannungsstern), lautet die allgemeine, hier zu lösende Aufgabe folgendermafen:

Gegeben ist ein System von $N$ Strahlen (entsprechend den N Nuten einer einschichtigen Wicklung), von denen sich je t (gemeinsamer Teiler von Nutenzahl und Polpaarzahl) decken, derart, daf je $N^{\prime}=N / t$ Strahlen einen regelmäfigen Stern bilden. Die N Strahlen sollen in m Gruppen (entsprechend den $m$ Wicklungssträngen) eingeteilt werden; die Zahl der Strahlen kann in jeder Gruppe verschieden, muf3 aber gerade sền (Mantelspulen). Die Strahlen jeder Gruppe sind so zusammenzusetzen, daf3 die $\mathrm{m}$ resultierenden Strahlen der $\mathrm{m}$ Gruppen einen regelmäfsigen Stern bilden (Symmetriebedingung). Bei der Zusammensetzung der Einzelstrahlen soll die Hälfte der Strahlen jeder Gruppe das negative Vorzeichen erhalten (Mantelspulen); gleichphasige Strahlen in derselben Gruppe dürfen nicht mit verschiedenem Vorzeichen auftreten (um die Unwirksamkeit von Spulen oder Spulenseiten auszuschliefien).

Es läft sich, wie mir meine Kollegen, die Herren Prof. Dr. Mohrmann und Privat. dozent Dr. Brandt, freundlichst bestätigt haben, zahlentheoretisch nachweisen, dafs diese Aufgabe nur dann gelöst werden kann, wenn die Gleichung (12a) und (12b) gelten. Diese Gleichungen stellen daher nicht nur die hinreichenden sondernauch die not. wendigen.Bedingungen für die Ausführbarkeit einer symmetrischen m-phasigen Wicklung mit einschichtigen Mantelspulen dar. 
Spannungen der beiden Wicklungsstränge gleich groß sind, ihr Phasenunterschied aber $90^{\circ}$ beträgt. Die bei der Zweiphasenwicklung um $180^{\circ}$ verschobenen Spannungen der Vierphasenwicklung brauchen nun nicht gleich groß zu sein; deshalb dürfen wir bei der Zweiphasenwicklung auch in Gl. I2a $\mathrm{m}=2$ setzen, während $\mathrm{Gl}$. I2 $\mathrm{b}$ übergeht in

$$
\frac{\gamma}{\mathrm{t}}=\operatorname{gan} z
$$

wenn wir für die $Z$ weiphasenwicklung wieder $\mathrm{m}=2$ (und nicht $\mathrm{m}=4$ ) setzen.

Die beiden Symmetriebedingungen lauten also:

erstens für alle Wicklungen

$$
\frac{\overline{\mathbf{N}}}{2 \mathrm{~m}}=\boldsymbol{\gamma}
$$

worin $\mathrm{m}$ die Phasen- oder Strangzahl ist;

zweitens für $\mathrm{Z}$ weiph asenwicklungen

$$
\frac{\gamma}{t}=\operatorname{ganz}
$$

für alle anderen Wicklungen

$$
\frac{2 \gamma}{\mathbf{t}}=\operatorname{ganz}
$$

Praktische Bedeutüng haben heute nur die Ein-, Zwei- und Dreiphasenwicklungen. Da nach unserer Voraussetzung alle Nuten bewickelt sein sollen, und die Einphasenwicklungen dadurch schlecht ausgenützt sind (Wicklungsfaktor etwa $2 / \pi$ ), wollen wir uns zunächst auf die Zwei- und Dreiphasenwicklungen beschränken.

Wenn wir $\gamma$ alle Zahlen des Zahlensystems durchlaufen lassen, so ergeben sich durch Multiplikation mit $2 \mathrm{~m}$ die Nutenzahlen, die der ersten, sowohl für Zwei- als für Dreiphasenwicklungen gültigen Symmetriebedingung I 2 a genügen. Diese Nutenzahlen sind in der zweiten und dritten Zeile des Kopfes der Tafel 2 eingeschrieben, worin die Spulenzahl $\gamma$ eines Strangs (I. Zeile) alle Zahlen von I bis 20 durchläuft. Dividieren wir $\gamma$ durch die Polpaarzahl, so erhalten wir die Nutenzahl für Pol und Strang

$$
q=\frac{\gamma}{p}=\frac{N}{2 p m}=g+\frac{z}{n}
$$

Diese Zahlen $\mathrm{q}$ sind unter die zugehörigen Nutenzahlen für Polpaarzahlen von 2 bis 20 , die an der linken Seite der Tafel angeschrieben sind, eingetragen. Ein Teil der Polpaarzahlen izt in eckige Klammern gesetzt, um sie von den Polpaarzahlen, die im Anhang zu den Maschinennormalien des Verband Deutscher Elektrotechniker empfohlen werden, zu unterscheiden. Die Tafel enthält also bis zu 20 Polpaaren alle Zweiphasenwicklungen bis zu 80 Nuten und alle Dreiphasenwicklungen bis zu 120 Nuten, deren Spulenzahl durch die entsprechende Strangzahl teilbar ist (GI. I 2 a). Die Wicklungen, deren Spulenzahl $\gamma$ eines Strangs durch die Polpaarzahl ganz teilbar ist $(\mathrm{q}=$ ganz $)$, ergeben Ganzlochwicklungen, die wir hier nicht weiter behandeln wollen.

Um die einander zugeordneten Nuten und Polpaarzahlen zu finden, die auch der zweiten Symmetriebedingung $12 \mathrm{~b}^{\prime} \mathrm{bzw}$. I $2 \mathrm{~b}$ genügen, suchen wir zunächst den größten Teiler $t$ auf, den die Nutenzahl $N$ und die Polpaarzahl $p$ gemeinsam haben. Diese Teiler $\mathrm{t}$ sind in Tafel 3 für Zweiphasenwicklungen, in Tafel 4 für Dreiphasenwicklungen eingeschrieben. Aus diesen Tafeln läßt sich ohne weiteres ablesen, welche zugeordneten Nuten- und Polpaarzahlen auch den Symmetriebedingungen I $2 b^{\prime}$ bzw. I 2 b genügen. Für die Zweiphasenwicklungen muß die einfache Spulenzahl eines Stranges, die in der ersten Zeile des Kopfes der Tafel angeschrieben ist, für die Dreiphasenwicklung die doppelte Spulenzahl eines Strangs durch $t$ teilbar sein. In den Tafeln 3 und 4 sind die Werte von $t$; deren zugehörige Nuten- und 


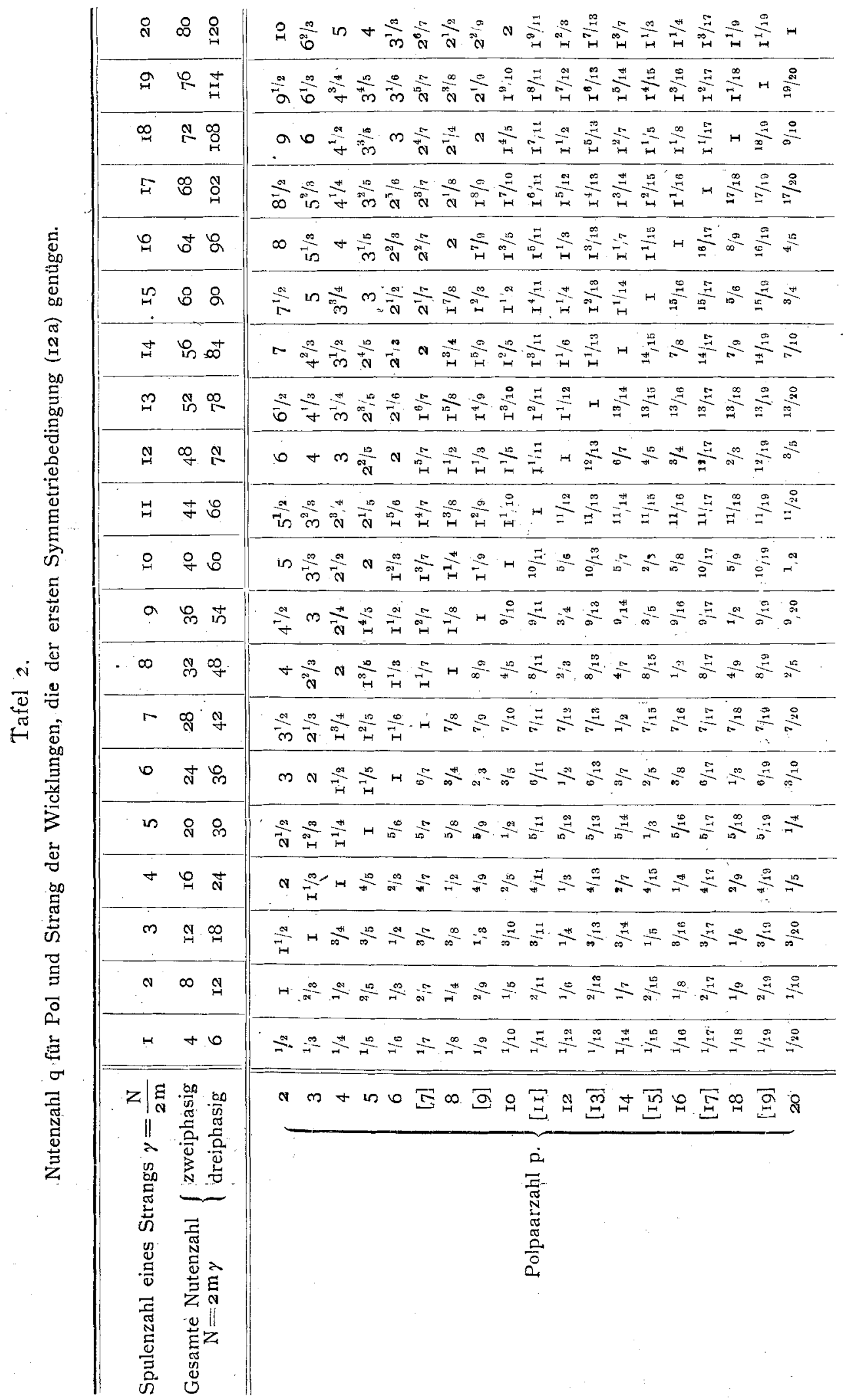


.

音

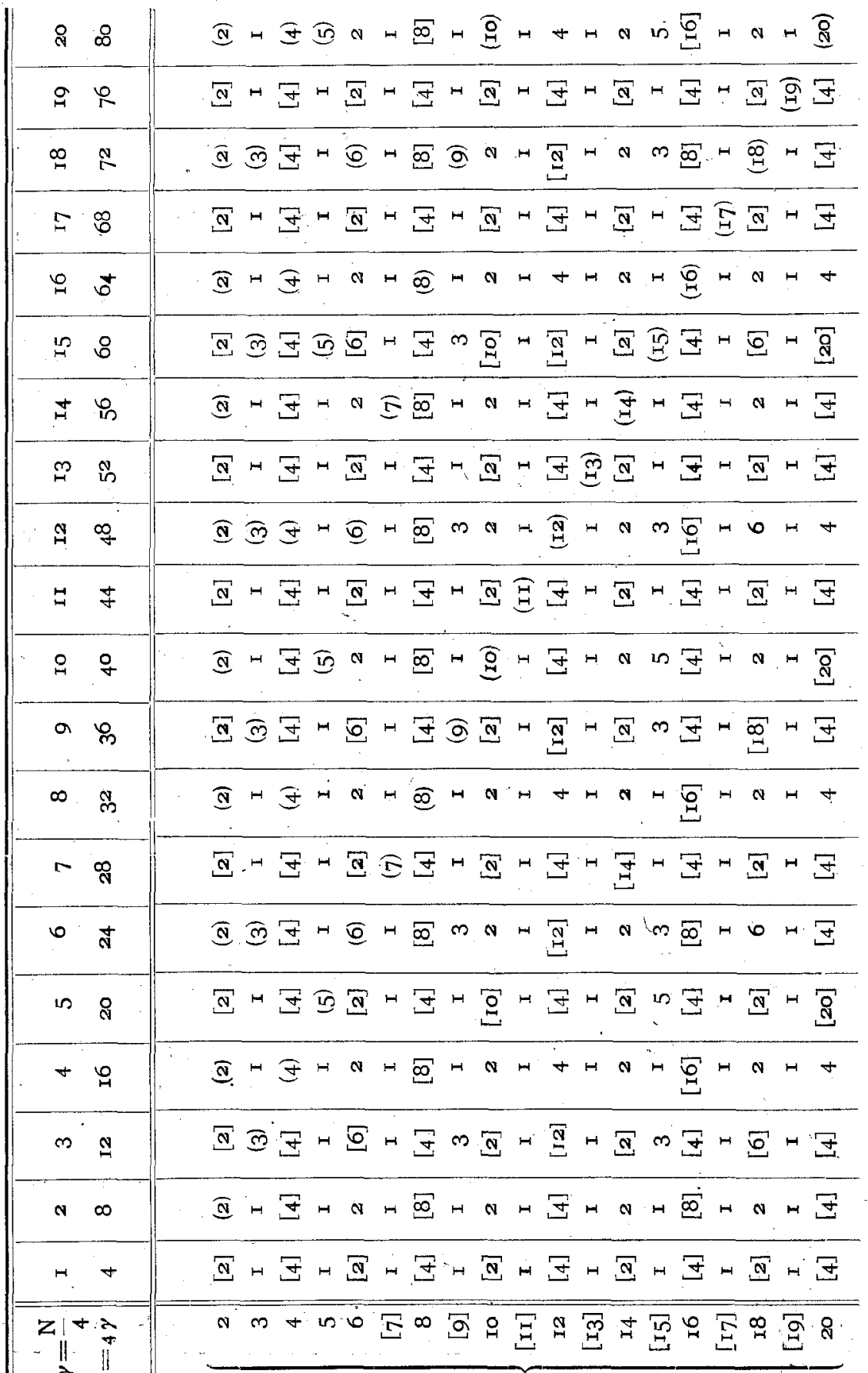

is $z$ 
$\Leftrightarrow$

?.

무

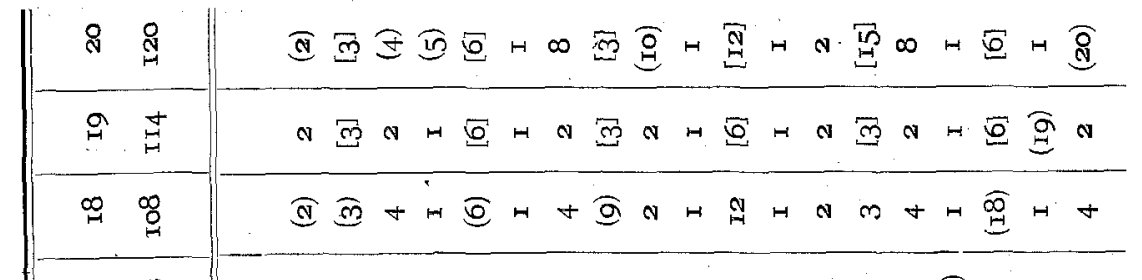

$\Xi$ \&

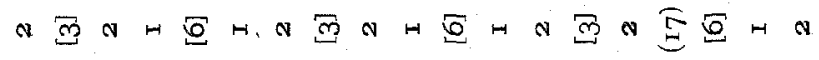

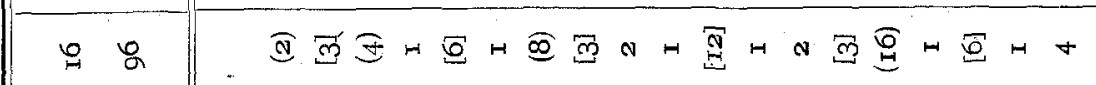

in 8

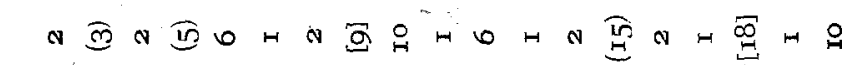

\begin{tabular}{|c|c|}
\hline$\underset{+}{+}+$ & 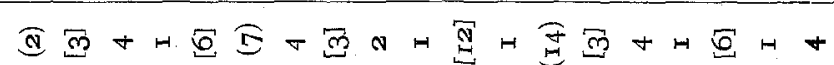 \\
\hline
\end{tabular}

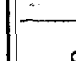

$\underset{r}{m} \infty$ ๙

i $N$

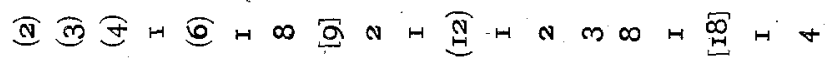

म 8 \%

\begin{tabular}{lll}
1 & 8 \\
\hline & 8
\end{tabular}

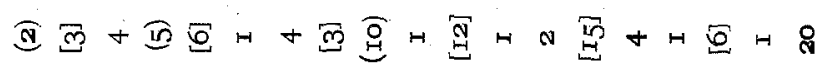

$\infty$ in

N $\widehat{3}$ N

저요

\begin{tabular}{|c|c|}
\hline$r$ & 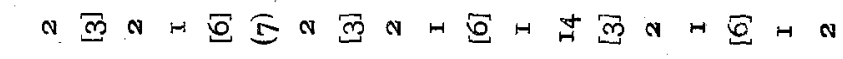 \\
\hline
\end{tabular}

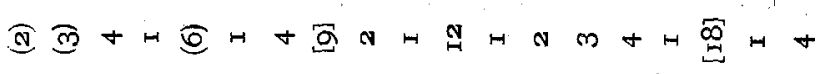

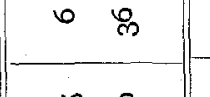

๙

\begin{tabular}{|c|c|c|}
\hline$+\delta$ & aे & 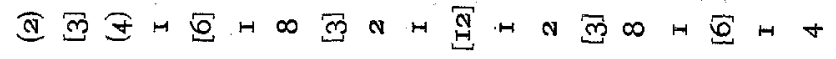 \\
\hline$m a$ & $\underset{\sim}{\infty}$ & 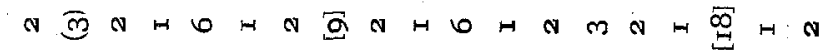 \\
\hline N & 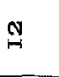 & 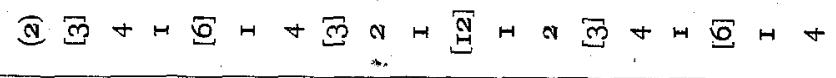 \\
\hline$+\quad$ ic & ○ & 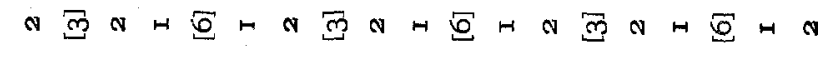 \\
\hline 210 & & * \\
\hline 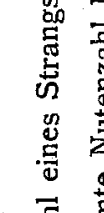 & 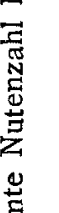 & 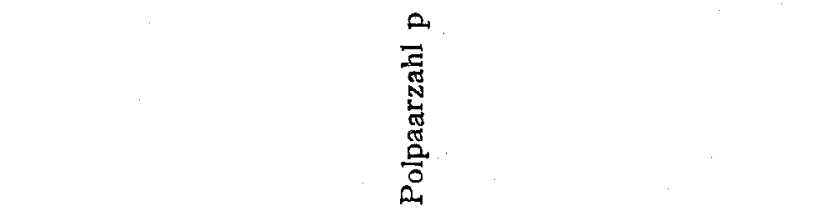 \\
\hline
\end{tabular}




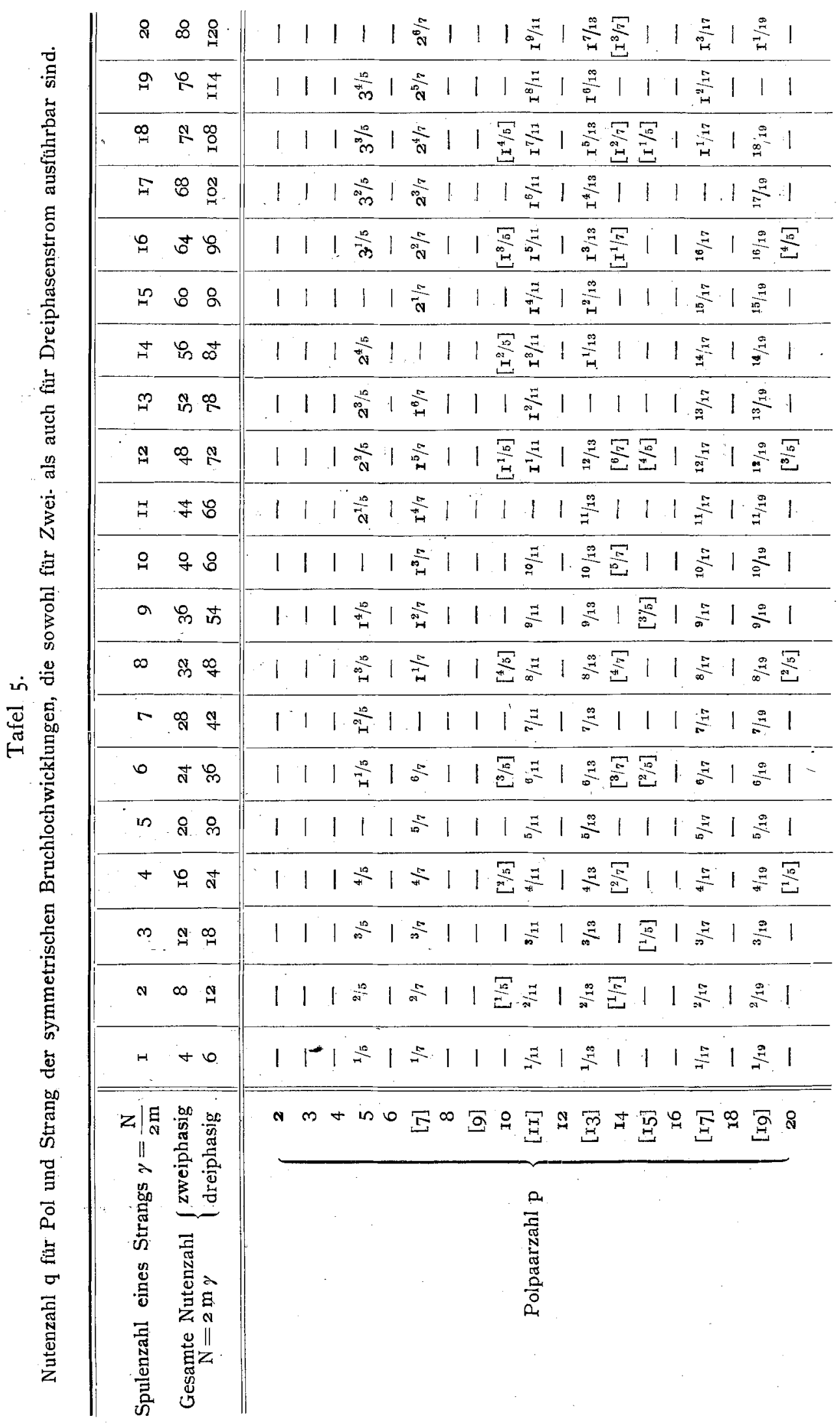




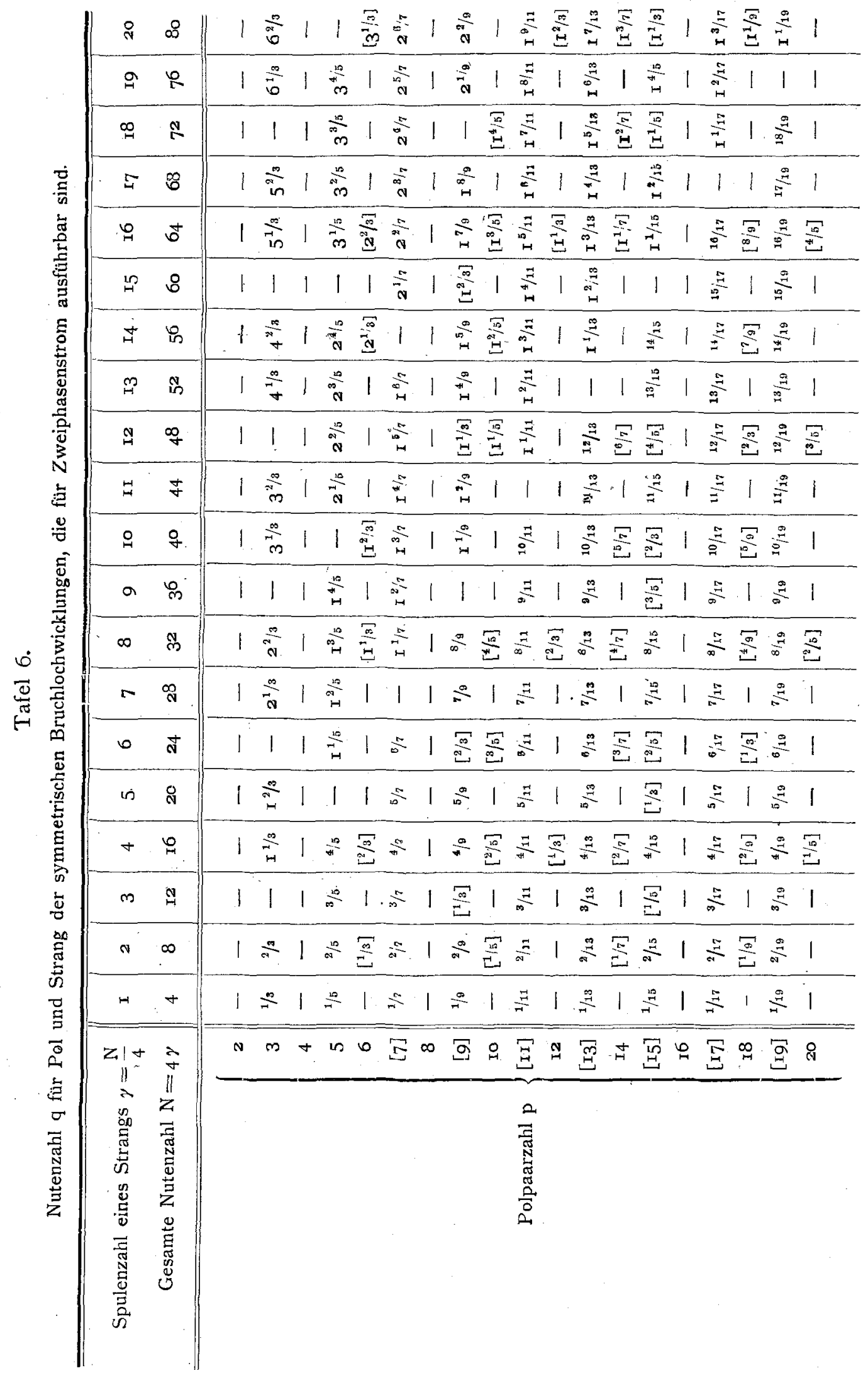




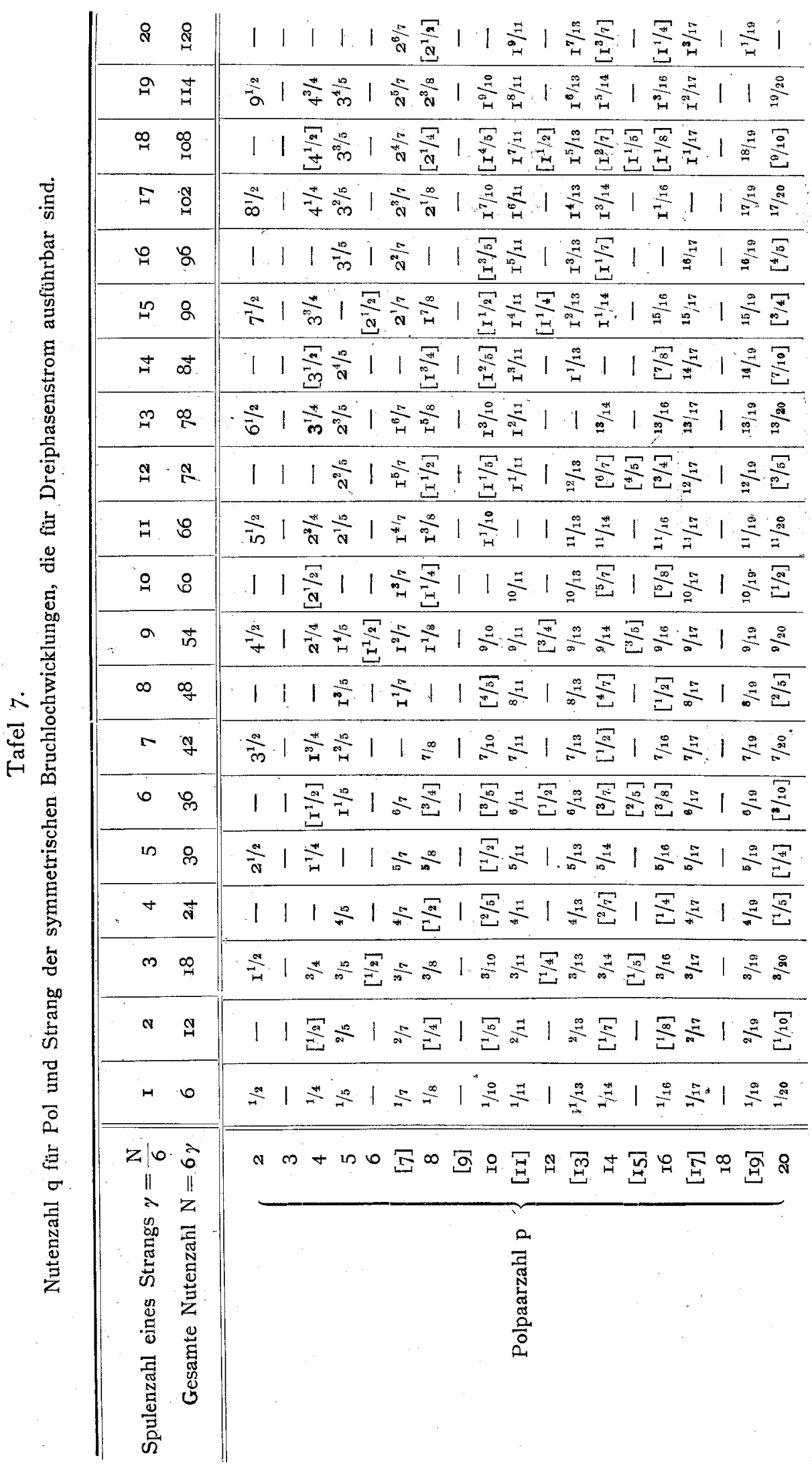


Polpaarzahlen den Symmetriebedingungen I $2 b^{\prime}$ bezw. I $2 \mathrm{~b}$ n icht genügen, in eckige Klammern, die den Ganzlochwicklungen entsprechenden Werte von $t$ in runde Klammern gesetzt.

In den Tafeln 5, 6 und 7 sind schließlich die Nutenzahlen q für Pol und Strang mit den zugehörigen Nuten- und Polpaarzahlen der symmetrischen Bruchlochwicklungen zusammengestellt. Tafel 5 enthält die Wicklungen, die sowohl für Zweials auch für Dreiphasenstrom, Tafel 6 die Wicklungen, die für Zwei- und Tafel 7 die, die für Dreiphasenstrom ausführbar sind. Die Bedeutung der eingeklammerten Zahlen von $\mathrm{q}$ in diesen Tafeln werden wir später kennen lernen. Die Tafeln 5, 6 und 7 lassen sich natürlich zu einer einzigen vereinigen, wenn man die Wicklungen, die für Zwei- und für Dreiphasenstrom ausführbar sind, durch verschiedene Farben oder Zeichen unterscheidet.

Wir haben in den Tafeln nur die Nutenzahlen bis I20 berücksichtigt, weil aus der gesetzmäßigen Folge der Zahlen von $q$ sich ohne weiteres die Wicklungen für größere Nutenzahlen ergeben.

4. Symmetrische Bruchlochwicklungen, deren Nutenzahl (N) ind Polpaarzahl $(p)$ keinen gemeinsamen Teiler haben $(t=I)$. Wenn $N$ und $p$ keinen gemeinsamen Teiler haben, so sind nach Gl. I2 a auch $\gamma$ und $p$ teilerfremd. Die Nutenzahl für Pol und Strang läßt sich deshalb immer in der Form

$$
\mathrm{q}=\mathrm{g}+\frac{\mathrm{z}}{\mathrm{p}}
$$

schreiben, worin $g$ eine beliebige ganze Zahl (einschließlich null) ist und $z$ und $p$ nicht gekürzt werden können. Der Gl. I3 entsprechen bei Zweiphasenstrom mit Rücksicht auf Gl. I2 $\mathrm{b}^{\prime}$ nur die Wicklungen, die in diesem Abschnitt behandelt werden, deren Nutenzahl $N$ und Polpaarzahl p also teilerfremd sind $(t=I)$, bei Dreiphasenstrom aber mit Rücksicht auf Gl. I2b auch noch die Wicklungen mit ungerader Spulenzahl $\gamma$, deren Nutenzahl und Polpaarzahl den größten gemeinsamen Teiler $\mathrm{t}=2$ haben.

In den Tafeln 5, 6 und 7 sind die Werte von $q$ für alle Bruchlochwicklungen, deren Nutenzahl für Pol und Strang in Form von Gl. I 3 geschrieben werden kann, ohne Klammern gedruckt. Von den Dreiphasenwicklungen werden wir die mit 'geraden Polpaarzahlen erst im nächsten Abschnitt behandeln; denn da nach Gl. I 2 a $\mathrm{N}$ immer gerade sein muß, scheiden bei den Wicklungen dieses Abschnitts alle geraden Polpaarzahlen aus. Bei Dreiphasenwicklungen $(\mathrm{m}=3)$ scheiden außerdem alle Polpaarzahlen aus, die durch 3 teilbar sind.

Ausführbare Polpaarzahlen sind bei $Z$ we iphasenstrom:

I. Alle Primzahlen mit Ausnahme von 2, also $\mathrm{p}=3,5,7$, I I, I3, I7, I9 usw.

2. Die ungeraden Zahlen, die mit $\gamma$ keinen gemeinsamen Teiler haben; also

$$
\begin{aligned}
& \mathrm{p}=9 \text { und } \gamma=\mathrm{I}, 2,4,5,7 \text { usw., } \\
& \mathrm{p}=15 \text { und } \gamma=\mathrm{I}, 2,4,7,8 \text { usw. }
\end{aligned}
$$

Ausführbare Polpaarzahlen sind bei Dreiphasenstrom:

r. Alle Primzahlen mit Ausnahme von 2 und 3 , also $p=5,7,1$ I, 1 3, 17, 19 usw.

2. Die ungeraden und nicht durch 3 teilbaren Zahlen, die mit $\gamma$ keinen gemeinsamen Teiler haben. Dieser Fall kommt nur bei sehr hohen Polpaarzahlen vor, wie sie in der Praxis selten ausgeführt werden. Die niedrigste Polpaarzahl ist $\mathrm{p}=25$, hierfür ist die. Wicklung mit $\gamma=\mathbf{I}, 2$, $3,4,6$ usw. ausführbar. -

Bei der Aufzeichnung des Spannungssterns haben wir zu beachten, daß $t=I$ ist. Nuten gleicher Phase kommen also hier nicht vor, und die Spannungen der Spulenseiten bilden einen regelmäßigen Stern mit N Strahlen. Dieser Spannungssstern stimmt mit einem der p Spannungssterne einer Ganzlochwicklung überein, 
deren gesamte Nutenzahl pN, oder deren Nutenzahl für Pol und Strang gleich $\gamma$ ist. Es müssen deshalb bei ungerader Spulenzahl im Strang $(\gamma=$ ungerade) alle Spulen in Reihe geschaltet werden, während bei gerader Spulenzahl im Strang ( $\gamma=$ gerade) immer zwei parallele Wicklungszweige möglich sind. Benächbarte Strahlen schließen im Spannungsstern hier immer den Winkel (Gl. 6')

$$
\alpha^{\prime}=\frac{\mathrm{I}}{\mathrm{N}} 360^{\circ}
$$

ein, während der Phasenwinkel zwischen benachbarten Nuten nach Gl. 6

$$
\alpha=\frac{\mathrm{p}}{\mathrm{N}} 360^{\circ}=\mathrm{p} \alpha^{\prime}
$$

ist. Die Phasen unmittelbar aufeinanderfolgender Nuten liegen im Spannungsstern also immer um p Strahlen auseinander.

Den Entwurf der Spannungssterne und der Wicklung wollen wir an einer D reiphasenwicklung mit $\mathrm{N}=24$ Nuten und $\mathrm{p}=5$ Polpaaren näher erläutern. Die Nutenzahl q für Pol und Strang ist

$$
\mathrm{q}=\frac{\mathrm{N}}{2 \mathrm{p} \mathrm{m}}=\frac{4}{5}
$$

Der Nutenstern ist in Abb. 6a dargestellt. Wir erhalten $N=24$ gleichmäßig verteilte Strahlen, die den einzelnen Nuten des Ankers entsprechen. Wenn die Nuten so numeriert werden, wie sie am Ankerumfang aufeinander folgen, und wir den mit I bezeichneten Strahl der Nut I zuordnen, so finden wir die den Nuten 2, 3, 4 usw. entsprechenden Strahlen, indem wir immer um je $p=5$ Strahlen vorrücken, also immer je $\mathrm{P}-\mathrm{I}=4$ Strahlen im Nutenstern überspringen. Auf diese Weise ergeben sich die an die Strahlen des Nutensterns geschriebenen Zahlen, die den fortlaufend numerierten Nuten des Ankers entsprechen.

Wir haben nun die einzelnen Nuten den drei Wicklungssträngen zuzuteilen. Diese Zuteilung soll so erfolgen, daß die resultierenden Spannungen der Wicklungsstränge möglichst groß sind. Die Spannungen der gleichsinnigen Spulenseiten desselben Wicklungsstrangs müssen deshalb möglichst wenig Phasenunterschied aufweisen, die Spannungen der ungleichsinnigen Spulenseiten dagegen möglichst um $180^{\circ}$ in der Phase verschoben sein. Die Zahl der gleichsinnigen Spulenseiten eines Stranges ist

$$
\gamma=\frac{\mathrm{N}}{2 \mathrm{~m}}=4 \text {. }
$$

Wir erhalten die in $\mathrm{Abb} .6 \mathrm{a}$ angedeutete Verteilung der Nuten auf die einzelnen Wicklungsstränge. Die Spannungen der Spulenseiten sind hier wieder für die drei Wicklungsstränge durch voll ausgezogene, gestrichelte und punktierte Linien, positive und negative Spulenseiten durch verschiedene Strichstärke unterschieden. Die Spannungsstrahlen der negativen Spulen-

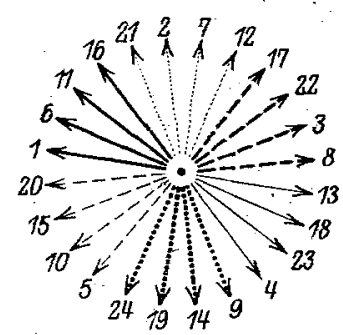

Abb. 6.a. Nutenstern einer dreiphasigen Bruchlochwicklung (Abschnitt 4 ) mit $\mathrm{N}=24, \mathrm{p}=5$, $\gamma=\dot{4}, \mathrm{q}=4$.

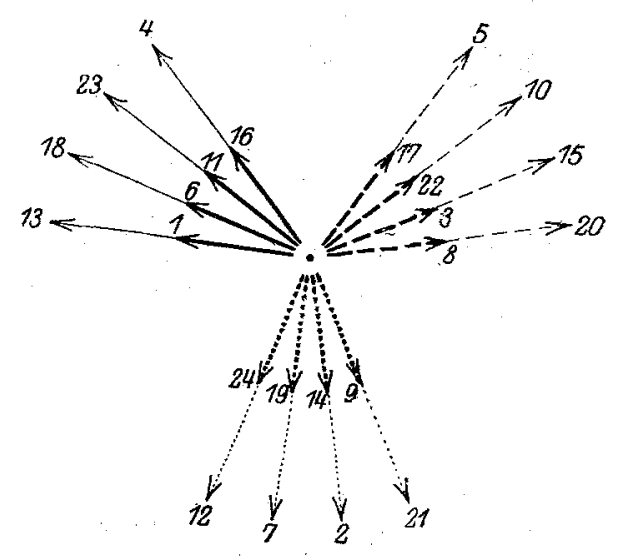

Abb. 6b. Einzelspannungen für die Wicklung zu. Abb. 6a. 
seiten werden in der fertigen Wicklung negativ (vgl. Abschnitt 2), so daß sich die resultierenden Spannungen der drei Wicklungsstränge aus den Einzelstrahlen in Abb. $6 \mathrm{~b}$ zusammensetzen.

Je eine positive Spulenseite muß nun mit je einer negativen desselben Wicklungsstrangs $\mathrm{zu}$ einer Spule vereinigt werden. Die Auswahl dieser Spulenseiten kann willkürlich erfolgen und ist nur eine Frage der technisch zweckmäßigsten Ausführung. Um mit möglichst wenig Wicklungsmetall auszukommen, wird man natürlich solche Spulenseiten zu Spulen vereinigen, die möglichst kurze Querverbindungen ergeben, deren Nuten also möglichst wenig am Ankerumfang auseinander liegen. Diese Spulenseiten überblickt man sofort, wenn die Nuten- oder Spulenseiten im abgewickelten Ankerumfang aufgezeichnet werden. So erhalten wir z. B. für den Wicklungsstrang, der durch voll ausgezogene Linien gekennzeichnet ist (vgl. Abb. 6a), die kürzesten Spulenquerverbindungen, wenn wir (vgl. Abb. 6c) die Spulenseiten in den Nuten I und 23,6 und 4, I I und 13,16 und is $\mathrm{zu}$ Spulen vereinigen.

Im allgemeinen wird man die Anordnung der Wicklungsköpfe in zwei Ebenen bevorzugen. Eine solche Ausführung ist in unserem Beispiel mit $\mathrm{q}<\mathrm{I}$ möglich, ohne daß Spulenköpfe von der einen in die andere Ebene abzukröpfen sind, wie es

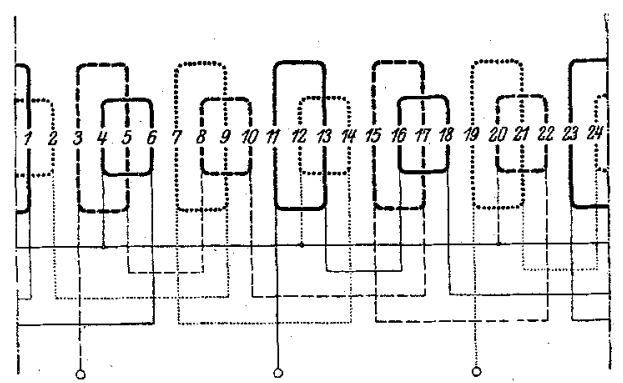

Abb. 6c. Schaltplan einer Bruchlochwicklung nach Abb. $6 a$ u. $b$ mit Reihenschaltung der Wicklungszweige; Sternschaltung der Wicklungsstränge.

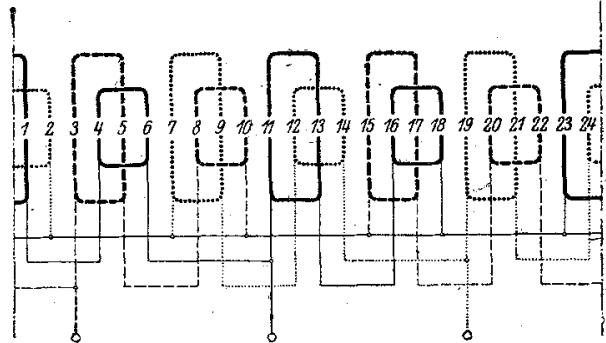

Abb. 6d. Schaltplan einer Bruchlochwicklung nach Abb. 6a u. b mit Parallelschaltung der Wicklungszweige ; Sternschaltung der Wicklungsstrănge.

bekanntlich alle Ganzlochwicklungen mit ungeraden Polpaarzahlen verlangen. Abb. $6 \mathrm{c}$ läßt die Spulenanordnung erkennen. Es ergeben sich für die ganze Wicklung nür zwei verschiedene Spulenformen. Alle Spulen haben dieselbe Weite und der Anker kann so geteilt werden, daß keine Spulenköpfe von der Teilungsebene geschnitten werden.

Die Schaltverbindungen zwischen den einzelnen Spulen desselben Wicklungsstrangs ergeben sich ebenfalls aus dem Spannungsstern in Abb. 6a, sie sind immer so zu legen, daß sie verschiedensinnige Spulenseiten miteinander verbinden. Die Reihenfolge der Spulen kann dabei beliebig sein und wird zweckmäßig so gewählt, daß die Verbindungsleitungen möglichst kurz werden. Legen wir, wie es beispielsweise in Abb. $6 \mathrm{c}$ geschehen ist, das aus Nut I I kommende Ende der Spule I I-I 3 an die Klemme der Maschine, so muß das aus Nut I 3 kommende Spulenende mit einem der Spulenenden in Nut I, 6 oder I6 verbunden werden, weil nach dem Spannungsstern in Abb. 6 a die Spulenseite 13 als negative Spulenseite gekennzeichnet und mit einer positiven zu verbinden ist. Wir schalten von Nut I3 nach I6, weil dies die kürzeste Verbindung ergibt. Ebenso lesen wir aus dem Spannungsstern die übrigen Spulenverbindungen des Schaltplans $6 c$ ab, in dem alle Spulen desselben Strangs in Reihe geschaltet sind. Im Schaltplan 6 c liegen die Wicklungsenden um $\mathrm{I}_{20^{\circ}}$ am Ankerumfang auseinander, die Wicklungsstränge sind beispielsweise in Stern geschaltet. Wenn die Klemmen der Wicklung nahe beieinander liegen sollen, wird man die aus den Nuten I I, I4 und I7 oder 8,9 und 
I 1 kommenden Spulenenden an die Maschinenklemmen legen und die Schaltverbindungen entsprechend abändern.

In unserm Beispiel ist $\gamma$ eine gerade Zahl, und die Spannungen je einer positiven und negativen Spulenseite sind phasengleich. Deshalb lassen sich in jedem Strang zwei Wicklungszweige parallel schalten. Nach dem Spannungsstern Abb. 6b ergeben die Spulen $I I-13$ und $16-18$ dieselbe resultierende Spannung nach Größe und Phase wie die Spulen $1-23$ und 6-4. Wir erhalten den Schaltplan in Abb. $6 \mathrm{~d}$ mit zwei parallelen Wicklungszweigen und Sternschaltung der Stränge.

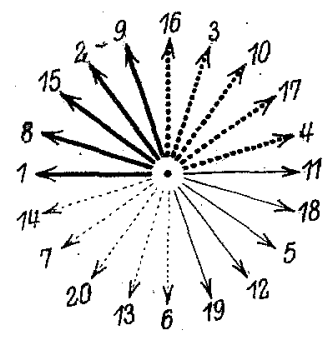

Abb. 7 a. Nutenstern einer zweiphasigen Bruchlochwicklung (Abschn. 4) mit $\mathrm{N}=\mathbf{2 0}$, $p=3, \gamma=5, q=1^{2} / 3$.

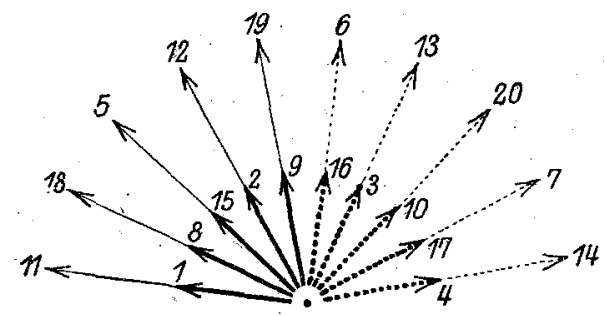

Abb. 7b. Einzelspannungen für die Wicklung zu Abb. $7 \mathrm{a}$.

Als Ausführungsbeispiel für eine $Z$ weiphasenwicklung, bei der $\mathrm{N}$ und $\mathrm{p}$ keinen gemeinsamen Teiler haben, wählen wir die Nutenzahl $\mathrm{N}=20$ und die Polpaarzahl $\mathrm{p}=3$. Es wird

$$
\mathrm{q}=\frac{\mathrm{N}}{2 \mathrm{pm}}=\mathrm{I}^{2} / 3
$$

Indem wir in entsprechender Weise wie bei der Dreiphasenwicklung verfahren, erhalten wir die Spannungssterne in Abb. $7 \mathrm{a}$ und $\mathrm{b}$. Abb. $7 \mathrm{c}$ stellt den abgewickelten

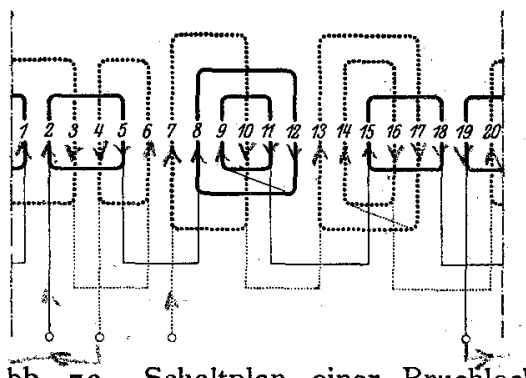

Abb. 7 c. Schaltplan einer Bruchloch. wicklung nach $\mathrm{Abb}$. $7 \mathrm{a}$ u. b. Reihenschaltung in den Wicklungssträngen.

Schaltplan dar. Es ergeben sich hier im ganzen Io Spulen, davon haben 6 verschiedene Form; alle Spulen desselben Wicklungsstranges müssen in Reihe geschaltet werden, weil $\gamma$ ungerade ist. Die Enden der beiden Wicklungsstränge sind $\mathrm{zu}$ besonderen Klemmen geführt.

Zum Entwurf der Wicklung ist die Aufzeichnung der Spannungssterne nicht immer erforderlich. Nebeneinander liegende Strahlen im Nutenstern enstprechen stets solchen Spulenseiten, die bei jeder Wicklung um dieselbe Anzahl (r) Nuten am Ankerumfang auseinander liegen. So ist z. B. im Nutenstern der Abb. 6a $r=5$ und in Abb. 7 a $r=7$. Den ,Nutenschritt“ $r$, um den wir am Ankerumfang oder im Schaltplan fortschreiten müssen, um zu benachbarten Strahlen im Nutenstern zu gelangen, können wir berechnen.

In den Nutensternen hatten wir die Strahlen, die um den „Sternschritt" p auseinander liegen, fortlaufend numeriert und damit die Phase der am Ankerumfang aufeinander folgenden Nuten erhalten. 'Benachbarte Nuten liegen also im Nutenstern um den Sternschritt $\mathrm{p}$ auseinander. Wenn wir daher am Ankerumfang den Nutenschritt $r$ ausführen, so entspricht dieser dem Sternschritt r p im Nutenstern. Dieser Sternschritt muss nun gleich dem um I vermehrten oder verminderten ganzen Vielfachen der Gesamtzahl (N) der Strahlen oder Nuten sein, damit uns der Schritt rp zu dem Nachbarstrahl im Nutenstern führt. Wir erhalten also für den Nutenschritt $r$ die Bedingung

$$
\mathbf{r} \mathbf{p}=\mathbf{g} \mathbf{N} \pm \mathbf{I}
$$


worin g eine beliebige ganze Zahl (einschließlich Null) ist. Wir bestimmen $r$ so, daß Gl. I4 mit dem kleinsten ganzzahligen Wert von g erfüllt ist. Für die Wicklung, die dem Nutenstern in Abb. 6a entspricht, ist diese kleinste ganze Zahl $\mathrm{g}=\mathrm{I}$ und wir erhalten

$$
\mathrm{r}=\frac{\mathrm{I} \cdot 24+\mathrm{I}}{5}=5 \text {. }
$$

Ebenso erhalten wir für Abb. 7 a (mit $\mathrm{g}=\mathrm{r}$ )

$$
\mathrm{r}=\frac{\mathrm{I} \cdot 20+\mathrm{I}}{3}=7
$$

Die Wicklung in Abb. $6 \mathrm{c}$ z. B. können wir nun, ohne den Nutenstern aufzu-' zeichnen, folgendermaßen entwerfen. Wir bestimmen zunächst die $\gamma=4$ positiven Spulenseiten des ersten Wicklungsstrangs, indem wir, beispielsweise mit der Nut I beginnend, um den Schritt $r=5$ fort. schreiten. Wir erhalten die Nuten I, 6, I I, I6. Die nächsten vier, je um den Schritt $\mathrm{r}=5$ auseinander liegenden Nuten gehören (vgl. Abb. 6a) den negativen Spulenseiten des dritten Wicklungsstrangs an, die darauf folgenden vier Nuten den positiven Spulenseiten des zweiten Strangs usw. Ungleichsinnige Spulenseiten desselben Wicklungsstrangs werden dann zu Spulen vereinigt. Die Spulen sind so zu schalten, dass immer positive Spulenseiten mit negativen verbunden werden, und umgekehrt.

Beim Entwurf sämtlicher, auch der im zweiten Teil dieser Arbeit behandelten Wicklungen, deren Nutenzahl $\mathrm{N}$ und Polpaarzahi $p$ teilerfremd sind, wird $m$ a $n$ den Nutenstern nicht erst aufzeichnen, sondern nach Gl. I 4 den Nutenschritt $r$ berechnen und die verschiedenen Spulenseiten der Wicklung unmittelbar im Schaltplan eintragen. Bei den Wicklungen, deren Nutenzahl $N$ und Polpaarzahl p einen gemeinsamen Teiler haben $(t>I, A b$ schnitt 5 und IO), zeichnet man jedoch zweckmäßig den Nutenstern auf, um die verschiedenen technischen Ausführungsmöglichkeiten leichter überblicken $\mathrm{zu}$ können.

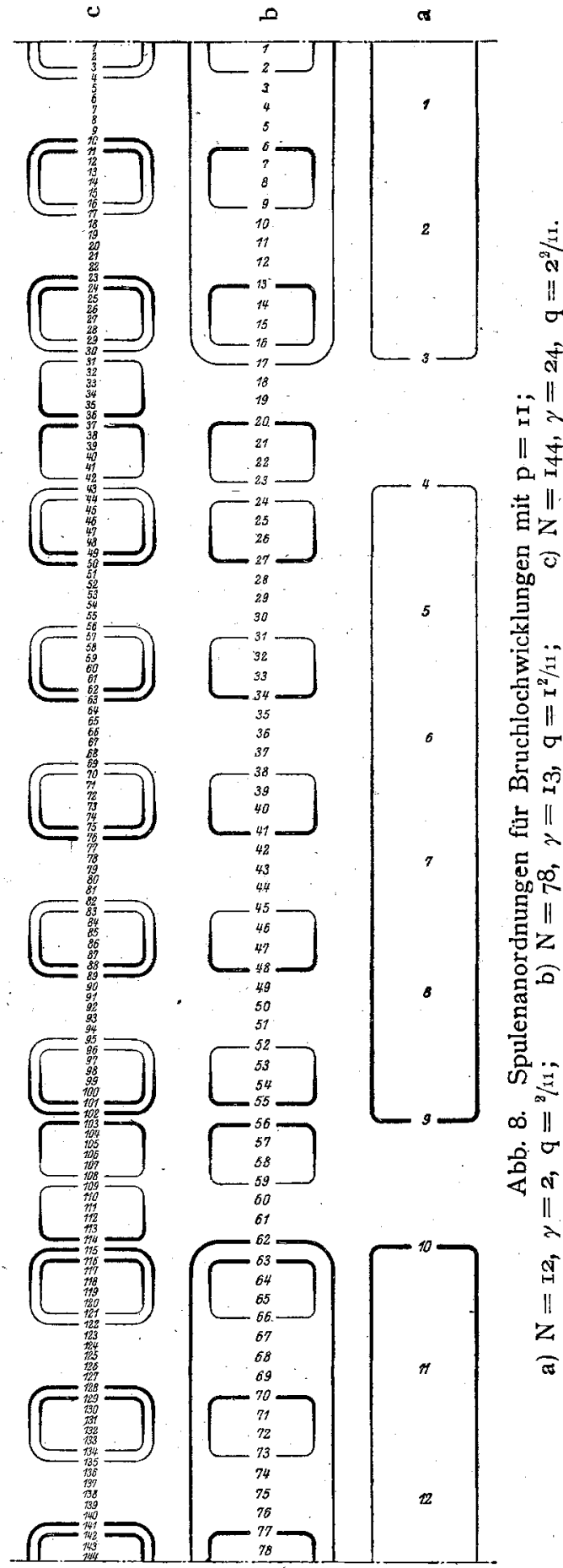

Bei großer Polpaarzahl p und kleiner Nutenzahl q für Pol und Strang lassen sich die Spulenseiten gewöhnlich nur so zu Spulen vereinigen, daß ein Teil der Querverbindungen wesentlich länger als eine Polteilung wird. Betrachten wir $z$. B. die 22-poligen Wicklungen $(p=I I)$ mit 
$\mathrm{N}=\mathrm{I} 2,78$ und $\mathrm{I} 44$
Nuten, entsprechend den Nutenzahlen für Pol und Strang

und den Spulenzahlen im Strang

$$
q=2 / 11, I^{2} / 11 \text { und } 2^{2} / 11
$$

$$
\gamma=2 \text {, I } 3 \text { und } 24 \text {. }
$$

Die Spulen je eines Wicklungsstrangs sind in Abb. $8 \mathrm{a}, \mathrm{b}$ und $\mathrm{c}$ dargestellt. Für diese drei Wicklungen ist nach Gl. 14 mit $\mathrm{g}=\mathrm{I}$

$$
\mathrm{r}=\mathrm{I}, 7 \text { und } \mathrm{I} 3 \text {. }
$$

Die positiven Spulenseiten sind für die erste Wicklung z. B. in die Nuten 9 und ro, für die zweite in die Nuten 6, I 320 usw., für die dritte Wicklung in die Nuten IO, 23, 36 usw. zu legen; sie sind durch dicke Linien hervorgehoben. Die negativen Spulenseiten desselben Wicklungsstrangs sind um $3 \gamma \mathrm{r}$ Nuten gegenüber den positiven verschoben, wobei wir nur die Nuten zu zählen brauchen, die den Rest von ganzen Vielfachen der gesamten Nutenzahl N bilden; dieser Rest ist hier $\frac{\mathrm{N}}{2}$. In den $\mathrm{Ab}$ bildungen sind die negativen Spulenseiten durch dünnere Linien bezeichnet. Bei der Wicklung mit $q=2 / \mathrm{I} \mathrm{I}$ (Abb. $8 \mathrm{a}$ ) ergeben sich in jedem Wicklungsstrang zwei lange Spulen. Bei der Wicklung mit $\mathrm{q}=\mathrm{I}^{2} / 1 \mathbf{1}$ (Abb. $8 \mathrm{~b}$ ) lassen sich die Spulenseiten so zu Spulen vereinigen, daß nur eine lange Spule erforderlich ist, während bei der dritten Wicklung (Abb. 8c) keine Spule wesentlich länger als die Polteilung ist.

Wicklungsfaktor. Die Spannungen der einzelnen Spulenseiten haben bei den in diesem Abschnitt behandelten Bruchlochwicklungen dieselbe Lage im Spannungsstern wie die Spulenseiten eines Polpaares einer Ganzlochwicklung mit $\mathrm{q}=\gamma$ Nuten für Pol und Strang (vgl. z. B. Abb. 3a und 6a). Der Wicklungsfaktor der $\nu \stackrel{\text { ten }}{=}$ Welle ist deshalb nach Gl. Io

$$
\xi_{v}=\frac{\sin \nu \frac{90^{\circ}}{\mathrm{m}}}{\gamma \sin \frac{\nu}{\gamma} \frac{90^{\circ}}{\mathrm{m}}} .
$$

Auch für die dreiphasigen Bruchlochwicklungen können die Wicklungsfaktoren der Táfel I entnommen werden, wenn wir darin $\mathrm{q}$ durch $\gamma$ ersetzen. Bei der Bruchlochwicklung ist nun $\gamma$ im allgemeinen wesentlich größer als die Nutenzahl q für Pol und Strang einer Ganzlochwicklung mit ungefähr derselben gesamten Nutenzahl N. Deshalb sind die Wicklungsfaktoren der Oberwellen der Bruchlochwicklung. im allgemeinen merklich kleiner und die Spannungskurve nähert sich mehr der Sinusform als bei der Ganzlochwicklung.

Zur Beurteilung der durch die Nutung des Ankers hervorgerufenen Oberwellen in der resultierenden E.M.K. müssen wir beachten, daß die Ordnungszahlen dieser Oberwellen in der Nähe von

$$
\nu_{\mathrm{N}_{1}}=\frac{\mathrm{N}}{\mathrm{p}}, \nu_{\mathrm{N}_{2}}=2 \frac{\mathrm{N}}{\mathrm{p}}, \ldots \ldots \nu_{\mathrm{Nn}}=\mathrm{n} \frac{\mathrm{N}}{\mathrm{p}}
$$

liegen. Dabei sind erfahrungsgemäß die Oberwellen zweiter Art (Ordnungszahl in der Nähe von $\nu_{\mathrm{N}_{2}}$ ) klein gegenüber den Oberwellen erster Art (Ordnungszahl in der Nähe von $\nu_{\mathrm{N}_{1}}$ ), während die Oberwellen noch höherer Art sich kaum bemerkbar machen.

Für die Oberwellen der Nutung $n \stackrel{\text { ter }}{=}$ Art ist nach Gl. 6 die gegenseitige Phasenverschiebung zwischen zwei am Ankerumfang nebeneinander liegenden Spulenseiten nahezu $\mathrm{n} 360^{\circ}$, während der 'Phasenwinkel benachbarter Strahlen im Nutenstern für die Oberwellen $n \stackrel{\text { ter }}{=}$ Art nahezu

$$
\beta_{\mathrm{n}}=\mathrm{n} \frac{360^{\circ}}{\mathrm{p}}
$$

ist. Beschränken wir uns auf die Oberwellen, für die $n<p$ ist, so wäre die resul- 
tierende Amplitude der Oberwellen im Wicklungsstrang nahezu null, wenn die Zahl der im Nutenstern nebeneinander liegenden Strahlen desselben Wicklungsstranges gleich der Polpaarzahl $\mathrm{p}$ oder einem Vielfachen davon wäre. In diesem Falle $\left(\frac{\gamma}{p}=q=\right.$ ganz) erhielten wir aber eine Ganzlochwicklung; nach Gl. I2 a müßte die Nutenzahl $\mathrm{N}$ durch die Polpaarzahl $\mathrm{p}$ teilbar sein, während wir für die Wicklungen in diesem Abschnitt vorausgesetzt haben, daß $N$ und $p$ teilerfremd sind. Wir erhalten im allgemeinen die kleinsten resultierenden Amplituden der Oberwellen, wenn sich die Zahl $q=\frac{\gamma}{p}$ möglichst wenig von einer ganzen Zahl unterscheidet, wenn also

$$
\mathrm{q}=\mathrm{g} \mp \frac{\mathrm{I}}{\mathrm{p}}
$$

ist, worin $g$ eine beliebige ganze Zahl ist. In diesem Falle ist, wie sich leicht übersehen läßt, die resultierende-Oberwelle von je $\gamma$ Spulenseiten eines Wicklungsstranges immer nahezu gleich der Oberwelle einer einzelnen Spulenseite. In der resultierenden E.M.K. eines Wicklungsstranges kommt daher von der Amplitude jeder Oberwelle der Nutung nur etwa

$$
\mathrm{v}=\frac{\mathbf{I}}{\gamma}
$$

zur Wirkung.

In den Wicklungen nach Abb. $6 \mathrm{c}$ und $\mathrm{d}$ z. B. ist der Phasenwinkel (vgl. Abb. 6a)

$$
\beta_{i}=\frac{360^{\circ}}{5}=72^{0}
$$

die Zahl der Nuten für Pol und Strang ist

$$
\mathrm{q}=\mathrm{I}-\frac{\mathrm{I}}{5}=\mathbf{4} / \mathrm{s}
$$

Die einzelnen Amplituden der Oberwellen erster Art von je vier in Reihe geschalteten Spulenseiten werden annähernd durch den Stern in

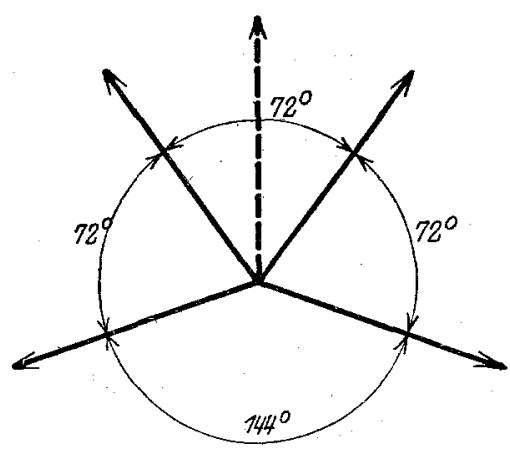

Abb. 9. Spannungsstern für die von der Nutung des Ankers herrührenden Oberwellen erster Art bei den Wicklungen nach Abb. 6 a bis d. Abb. 9 dargestellt. Die Resultierende ist gleich dem negativen fünften Strahl, de die übrigen vier zu Null ergänzt; sie ist in der Abbildung gestrichelt gezeichnet.

5. Symmetrische dreiphasige Bruchlochwicklungen, deren Nutenzahl (N) und Polpaarzahl (p) als größten gemeinsamen Teiler 2 haben $(t=2)$, und deren Spulenzahl im Strang ungerade ist $(\gamma=$ ungerade). Auch bei den Bruchlochwicklungen dieses Abschnitts müssen $\gamma$ und p teilerfremd sein, weil nach Gl. 12 a $\mathrm{N}=2 \mathrm{~m} \gamma$ immer gerade ist, und der größte gemeinsame Teiler von $\mathrm{N}$ und $\mathrm{p}$ hier 2 sein soll. Die Nutenzahl für Pol und Strang läßt sich deshalb wieder in der Form

$$
\mathrm{q}=\mathrm{g}+\frac{\mathrm{z}}{\mathrm{p}}
$$

schreiben (vgl. S. 229). Mit denjenigen Wicklungen in Abschnitt 4, die ungerade Spulenzahl im Strang haben $(\gamma=$ ungerade) stimmen sie außerdem noch darin überein, daß alle Spulen desselben Wicklungsstranges immer in Reihe geschaltet werden müssen. Alle dreiphasigen Bruchlochwicklungen dieser Art, die nicht unter Abschnitt 4 fallen, gehören hierher. Sie sind in Tafel 4 durch die ungeraden Zahlen von $\gamma$ und die Polpaarzahlen mit dem Teiler $t=2$ gekennzeichnet, in Tafel 7 durch die nicht eingeklammerten Zahlen von q bei geraden Polpaarzahlen. Die Zweiphasenwicklungen nach Gl. I3, soweit sie mit Rücksicht auf die zweite Symmetriebedingung (Gl. I $2 \mathrm{~b}^{\prime}$ ) ausführbar sind, haben wir in Abschnitt 4 bereits behandelt. 
Da $t=2$ sein soll, scheiden hier alle ungeraden Polpaarzahlen aus, außerdem aber auch die durch 3 teilbaren Polpaarzahlen, weil $\mathrm{N}$ immer ein Vielfaches von 3 ist.

Ausführbare Polpaarzahlen sind bei Dreiphasenstrom:

I. Alle ganzen Potenzen von 2, also $\mathrm{p}=2,4,8,16$ usw. bei allen ungeraden Zahlen von $\gamma$.

2. Alle übrigen nicht durch 3 teilbaren geraden Zahlen bei solchen ungeraden Zahlen von $\gamma_{\text {; }}$ die mit $\mathrm{p}$ keinen gemeinsamen Teiler haben, also

$$
\begin{aligned}
& \mathrm{p}=10 \text { und } \gamma=\mathrm{I}, 3,7,9, \mathrm{II}, \mathrm{I} 3, \text { I } 7 \text { usw., } \\
& \mathrm{p}=\mathrm{I} 4 \text { und } \gamma=\mathrm{I}, 3,5,9, \mathrm{I} \text { I, I } 3, \text { I } 5 \text { usw., } \\
& \mathrm{p}=20 \text { und } \gamma=\mathrm{I}, 3,7,9, \text { I I, I3, I } 7 \text { usw. }
\end{aligned}
$$

Als Ausführungsbeispiel wählen wir eine Wicklung mit $p=2$ und $N=18$, also mit der Nutenzahl für Pol und Strang

$$
\mathrm{q}=\frac{\mathrm{N}^{\mathrm{m}}}{2 \mathrm{pm}}=\mathrm{I}^{1 / 2} \text {. }
$$

Da Nutenzahl und Polpaarzahl den gemeinsamen Teiler $t=2$ haben, erhalten immer zwei Nuten dieselbe Phase. Numerieren wir wieder die Nuten, so wie sie am Ankerumfang aufeinander folgen, so erhalten wir den Nutenstern in Abb. IOa, in dem benachbarte Strahlen auch benachbarten Nuten angehören, weil $t=p$ ist (vgl. Gl. 6 und $6^{\prime}$ ).

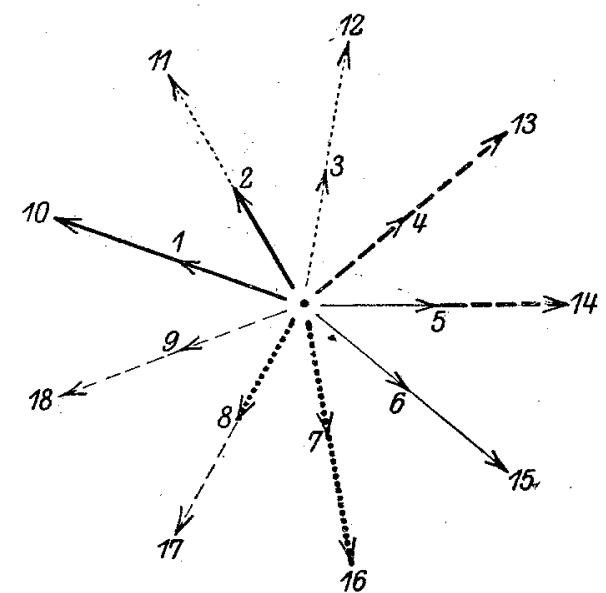

Abb. Ioa. Nutenstern einer (dreiphasigen) Bruchlochwicklung (Abschn. 5) mit $\mathrm{N}=18$, $\mathrm{p}=2, \gamma=3, \mathrm{q}=\mathrm{I}^{\mathbf{1}} / \mathbf{2}$

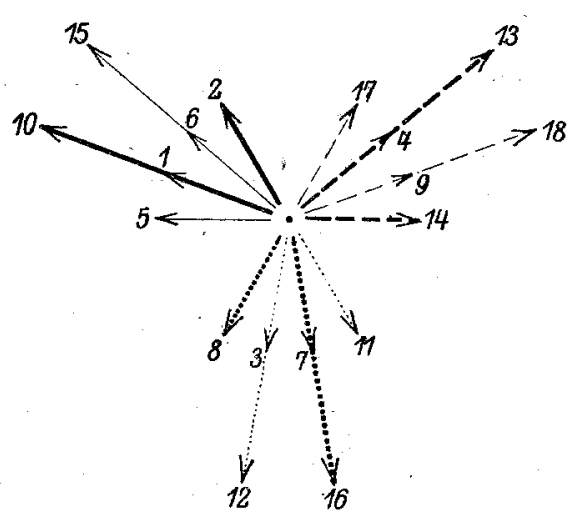

Abb. rob. Einzelspannungen für die Wicklung zu Abb. Ioa.

Die Zahl der gleichsinnigen Spulenseiten ist für jeden Strang

$$
\gamma=\frac{\mathrm{N}}{2 \mathrm{~m}}=3 \text {. }
$$

Damit die Wicklung möglichst gut ausgenützt wird, der Wicklungsfaktor also möglichst groß wird, sollen die gleichsinnigen Spulenseiten möglichst wenig Phasenunterschied aufweisen. Je zwei Nuten : haben nun immer dieselbe Phase, die Zahl der gleichsinnigen Spulenseiten ist aber ungerade, nämlich $\gamma=3$; deshalb muß je eine Spulenseite eines Wicklungsstranges mit der eines andern phasengleich sein. In $\mathrm{Abb}$. IOa sind dies die Spulenseiten in den Nuten 2 und I I, 5. und I4, 8 und I7. Für das Verhalten der Wicklung ist es vollkommen gleichgültig, welche der beiden gleichphasigen Nuten man dem einen oder andern Wicklungsstrang zuweist; man wird sie deshalb so auf die einzelnen Wicklungsstränge verteilen, daß die technisch zweckmäßigste und einfachste Wicklung entsteht. Dies läßt sich sehr schnell feststellen, wenn man im abgewickelten Schaltplan zunächst die Nuten, die nicht mehr willkürlich den Wicklungssträngen zugeordnet werden können, einzeichnet, wobei 
die drei Wicklungsstränge durch verschiedene Stricharten oder noch besser durch verschiedene Farben hervorzuheben sind. Die zweckmäßigste Aufteilung der übrigen Nuten auf die einzelnen Wicklungsstränge.läßt sich dann leicht feststellen, da hier nur drei Kombinationen möglich sind, die verschiedene Ausführungen ergeben. Bei einiger Übung kann man die zweckmäßigste Ausführung. schon aus dem Nutenstern ablesen, ohne daß man den abgewickelten Schaltplan aufzeichnet. Im Nutenstern, Abb. Ioa, sind die gleichphasigen Nuten so auf die Wicklungsstränge verteilt, daß die Spulenköpfe in zwei Ebenen angeordnet werden können, wie es der Schaltplan in Abb. Ioc erkennen läßt. Bei der Vereinigung von Spulenseiten zu Spulen und der Herstellung der Schaltverbindungen ist genau ebenso zu verfahren, wie es auf S. 23 I ausführlich erläutert ist. Wenn die ungleichsinnigen Spulenseiten zu

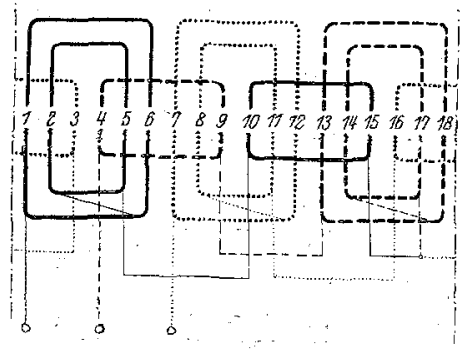

Abb, roc. Schaltplan einer Bruch. lochwicklung nach Abb. Ioa u. b. Reihenschaltung in den Strängen; Sternschaltung der Stränge. Spulen vereinigt werden, ändert sich natürlich wieder der Sinn der durch dünne Linien dargestellten Strahlen im Nutenstern, und wir erhalten in $\mathrm{Abb}$, Iob die Einzelspannungen, aus denen sich die Resultierenden der Strangspannungen zusammensetzen.

In den $\mathrm{Abb}$. I I $\mathrm{C}$ und $\mathrm{I} 2 \mathrm{c}$ sind noch dreiphasige Wicklungen derselben Polzahl $(\mathrm{p}=2)$ mit $\mathrm{q}$ gleich $1 / 2$ und $2^{1 / 2}$ und $\gamma$ gleich 1 und 5 dargestellt, die sich
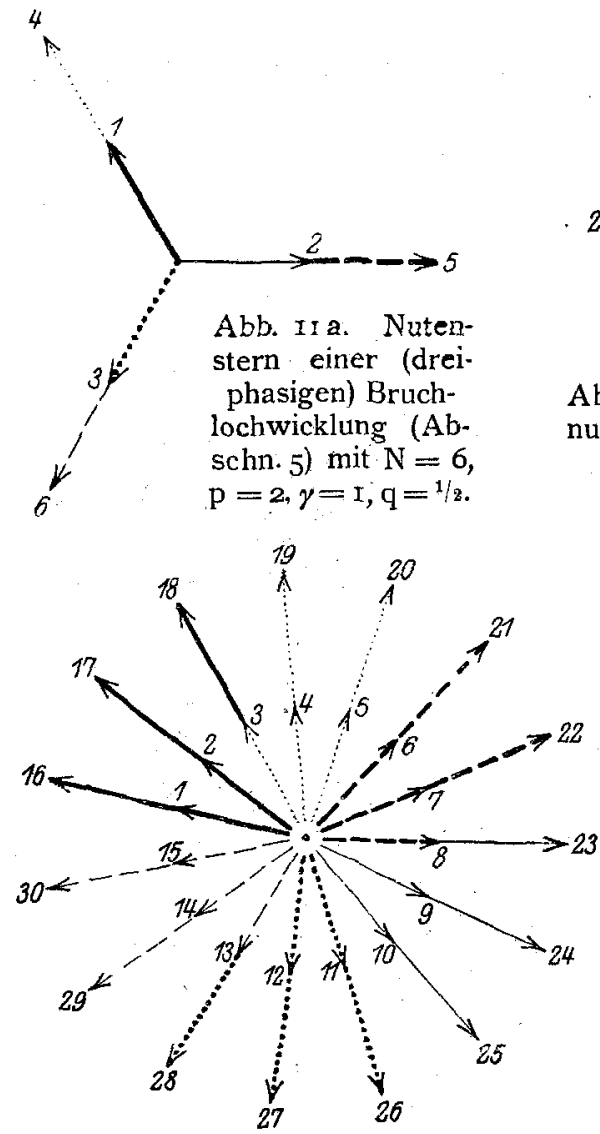

Abb. 12a. Nutenstern einer (dreiphasigen) Bruchlochwicklung (Abschn, 5) mit $\mathrm{N}=30$, $\mathrm{p}=2, \gamma=5, \mathrm{q}=2^{1 / 2}$.

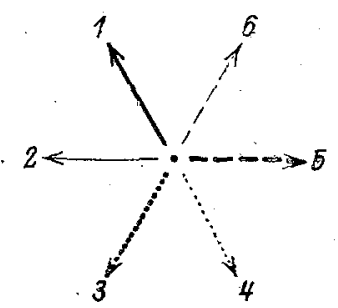

Abb. ir b. Einzelspannungen für die Wicklung zu Abb. Ira.

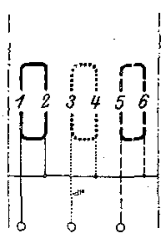

Abb. II c. Schaltplan einer Bruchlochwicklung nach Abb. II a und $b$. Sternschaltung d. Stränge (Spulen).

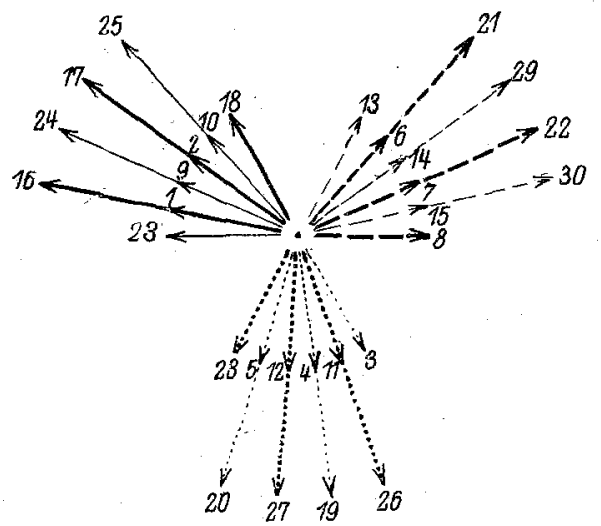

Abb. I2b. Einzelspannungen für die Wicklung zu Abb, I2a. 
aus den Nutensternen I I a und I 2 a ergeben. Die Abb. I I b und I 2 b stellen wieder die Einzelspannungen in den Wicklungssträngen dar.

Die Wicklungsköpfe aller vierpoligen $(\mathrm{p}=2)$ Bruchlochwicklungen dieses Abschnitts, deren Nutenzahl für Pol und Strang also in der Form

$$
q=g+\frac{1}{2}
$$

geschrieben werden kann, lassen sich in zwei Ebenen anordnen. Ihre Lage wird
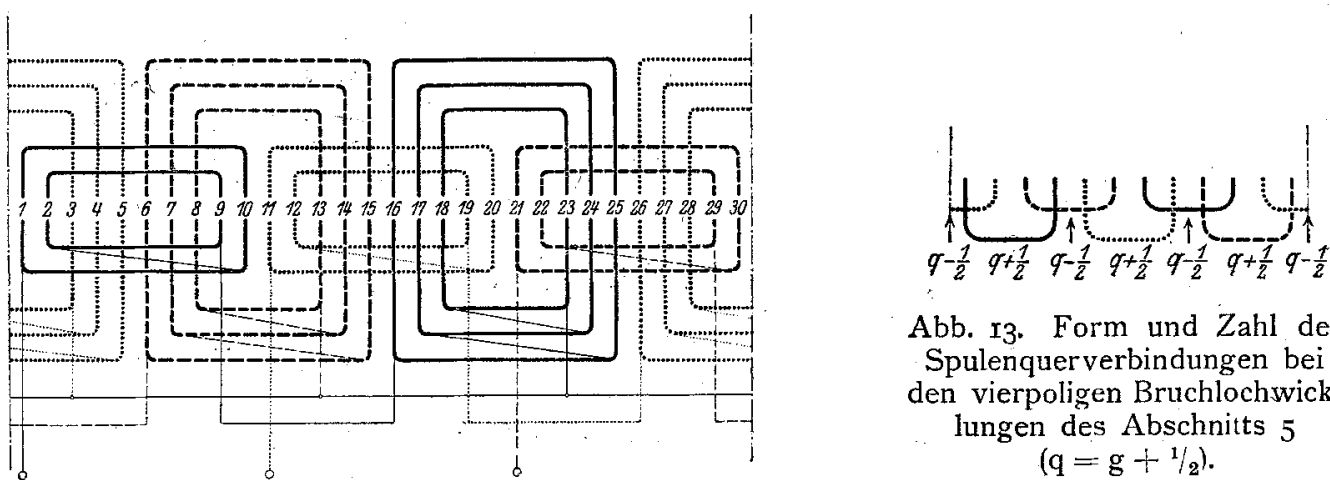

Abb. I3. Form und Zahl der Spulenquerverbindungen bei den vierpoligen Bruchlochwicklungen des Abschnitts 5 $(q=g+1 / 2)$.

Abb. I2c. Schaltplan einer Bruchlochwicklung nach Abb. 12a und b. Reihenschaitung in den Strängen; Sternschaltung der Stränge.

durch Abb. I3 dargestellt. Die Wicklungsköpfe erhalten abwechselnd $q-1 / 2$ und $\mathrm{q}+1 / 2$ Spulenquerverbindungen.

In Abb. I4 $\mathrm{c}$ ist auch .eine Bruchlochwicklung für $\mathrm{p}=4$ Polpaare aufgezeichnet. Die Gesamtzahl der Nuten ist $N=30$, die Nutenzahl für Pol und Strang $q=I^{1 / 4}$ und die Spulenzahl im Strang $\gamma=5$. Die Spannungssterne dieser Wicklung sind

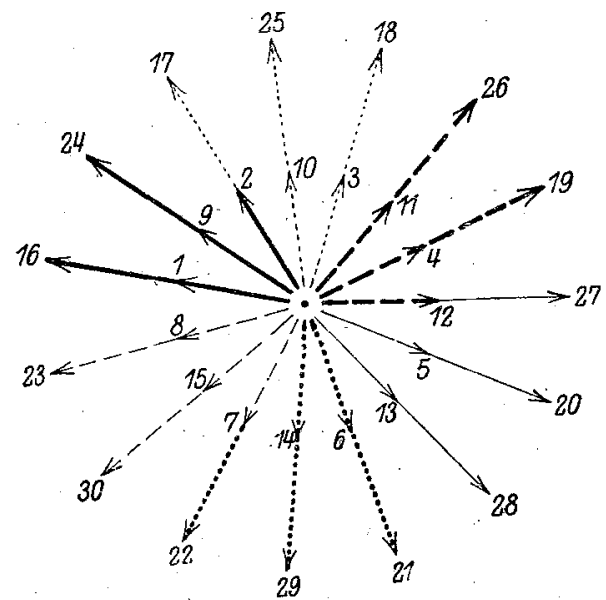

Abb. I4a. Nutenstern einer (dreiphasigen) Bruchlochwicklung (Abschn. 5) mit $\mathrm{N}=30$, $\mathrm{p}=4, \gamma=5, \mathrm{q}=\mathrm{I}^{1} / 4$.

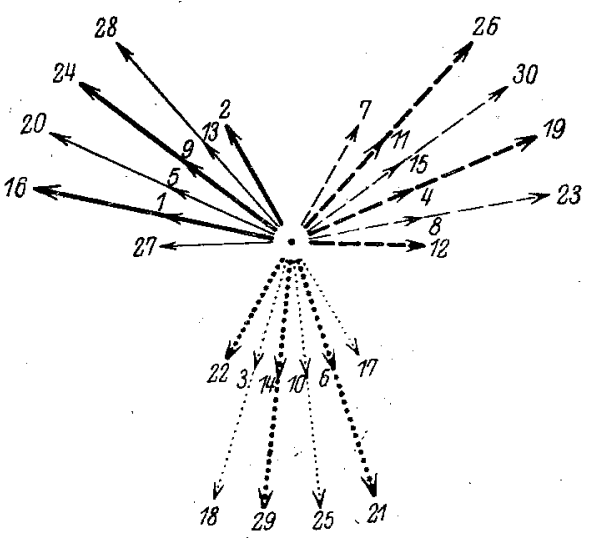

Abb. $\Upsilon_{4}$ b. Einzelspannungen für die Wicklung $\mathrm{zu} \mathrm{Abb}$. $\mathrm{I}_{4} \mathrm{a}$.

in Abb. $\mathrm{I}_{4} \mathrm{a}$ und $\mathrm{b}$ dargestellt. Sie unterscheiden sich von denen der vierpoligen Wicklungen mit derselben Spulenzahl im Strang (Abb. I2 a und b) nur durch die Numerierung der Strahlen. Bei vier Polpaaren ist $t=\frac{p}{2}$, deshalb liegen nach Gl. 6 und $\sigma^{\prime}$ im Nutenstern Abb. I4 a benachbarte Nuten um $\frac{\mathrm{p}}{2}=2$ ungleichphasige Strahlen 
auseinander. Die Wicklungsköpfe aller achtpoligen $(\mathrm{p}=4)$ Bruchlochwicklungen dieses Abschnitts, deren Nutenzahl für Pol und Strang also in der Form

$$
\mathrm{q}=\mathrm{g} \mp \frac{1}{4}
$$

geschrieben werden kann, lassen sich wieder in zwei Ebenen anordnen. Die Anzahl der Spulenquerverbindungen, die dann in einem Wicklungskopf nebenein ander liegen, ist in Abb. I 5 angeschrieben.

Die Wicklungsfaktoren der in diesem $\mathrm{Ab}$ schnitt behandelten Bruchlochwicklungen sind bei derselben Spulenzahl für Pol und Strang immer kleiner als bei den Wicklungen in Abschnitt 4. Da $t=2$ und $\gamma=$ ungerade, müssen sich die Einzelspannungen der gleichsinnigen Spulenseiten desselben Wicklungsstranges aus $\frac{\gamma-\mathrm{I}}{2}$ Gruppen von je zwei gleichphasigen und aús einem Einzelstrahl zu-

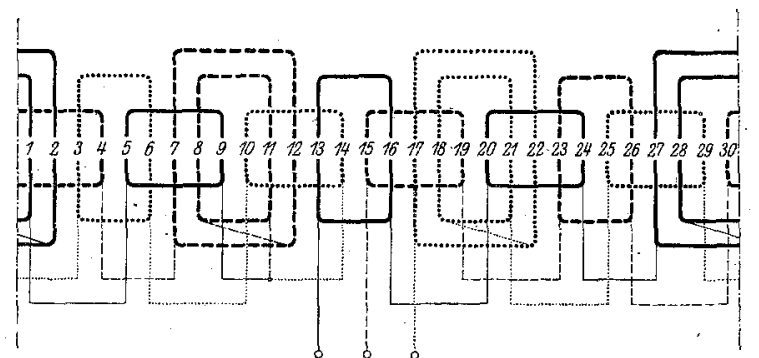

Abb. I4 c. Schaltplan einer Bruchlochwicklung nach Abb. $\mathbf{I}_{4} \mathrm{a}$ und $\mathrm{b}$. Reihenschaltung in den Strängen; Sternschaltung der Stränge.

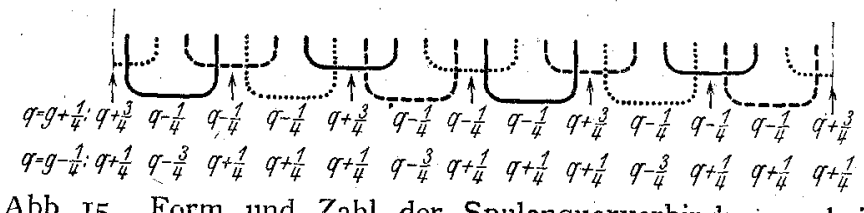

Abb. r. $_{5}$ Form und Zahl der Spulenquerverbindungen bei den achtpoligen Bruchlochwicklungen des Abschnitts 5 $(q=g \mp 1 / 4)$. sammensetzen; alle ungleichphasigen Strahlen sind um den Phasenwinkel (Gl. $\sigma^{\prime}$ )

$$
\alpha^{\prime}=\mathrm{t} \frac{360^{0}}{\mathrm{~N}}
$$

verschoben. Da ferner die Strangzahl $\mathrm{m}=3=$ ungerade, ist der Einzelstrahl gegenüber den Doppelstrahlen bei den negativen Spulenseiten in der Phase verfrüht, wenn er bei den positiven verspätet ist. Da schließlich $\frac{\mathrm{N}}{t}=3 \gamma$ hier immer ungerade ist, so sind die Spannungen der negativen Spulenseiten gegenüber denen der positiven immer um

$$
\frac{\alpha^{\prime}}{2}=\frac{\mathrm{I}}{2} \mathrm{t} \frac{360^{\circ}}{\mathrm{N}}=\frac{360^{\circ}}{\mathrm{N}}
$$

in der Phase verschoben. Wir erhalten also bei allen Wicklungen dieses Abschnitts, die durch die Abb. IOb bis $12 \mathrm{~b}$ und $\mathrm{I} 4 \mathrm{~b}$ gekennzeichnete Lage der 'Einzelspannungen.

Die Einzelspannungen der Spulenseiten, die in einem Wicklungsstrang liegen, lassen sich auch so in zwei Gruppen von je $\gamma$ Strahlen zusammenfassen, daß sowohl die Spannungen der beiden Gruppen als auch (wenn $\gamma>\mathrm{I}$ ) die Einzelspannungen jeder Gruppe um den Phasenwinkel

$$
\frac{\alpha^{\prime}}{2}=\frac{360^{\circ}}{\mathrm{N}}=\frac{60^{0}}{\gamma}
$$

gegeneinander verschoben sind. Für jede dieser Strahlengruppen ist nach G1. 8 mit der Wicklungsfaktor der $\boldsymbol{\nu}$ - $\stackrel{\text { ten }}{=}$ Welle

$$
S=\gamma \text { und } \varphi=\nu \frac{\alpha^{\prime}}{2}=\frac{\nu 60^{\circ}}{\gamma}
$$

$$
\xi_{\nu}^{\prime}=\frac{\sin \nu 30^{\circ}}{\gamma \sin \frac{\nu 30^{\circ}}{\gamma}}
$$




\section{Tafel 8.}

Verhältnis $\xi_{v}^{\prime \prime}$ zwischen den Wicklungsfaktoren der Bruchlochwicklungen nach den Abschnitten 5 und 4 bei derselben Spulenzahl $\gamma$ im Strang.

\begin{tabular}{|c|c|c|c|c|c|c|}
\hline \multirow[b]{2}{*}{$\gamma$} & \multicolumn{6}{|c|}{$\nu=$} \\
\hline & $\Upsilon$ & 3 & 5 & 7 & 9 & II \\
\hline I & 0,866 & 0 & 0,866 & 0,866 & 0 & 0,866 \\
\hline 2 & 0,966 & 0,707 & 0,259 & 0,259 & 0,707 & 0,966 \\
\hline 3 & 0,985 & 0,866 & 0,643 & 0,342 & 0 & 0,342 \\
\hline 4 & $0,99 I$ & 0,923 & 0,793 & 0,609 & 0,383 & 0,130 \\
\hline 6 & 0,996 & 0,966 & 0,906 & $0,8 \mathbf{r}_{4}$ & 0,707 & 0,574 \\
\hline IO & 0,999 & 0,988 & 0,966 & 0,934 & 0,891 & 0,839 \\
\hline 20 & 0,999 & 0,997 & 0,991 & 0,984 & 0,972 & 0,959 \\
\hline$\infty$ & $\mathbf{I}, 000$ & $\mathrm{I}, 000$ & 1,000 & 1,000 & 1,000 & 1,000 \\
\hline
\end{tabular}

Tafel 9 .

Wicklungsfaktoren der dreiphasigen Bruchlochwicklungen im Abschnitt 5.

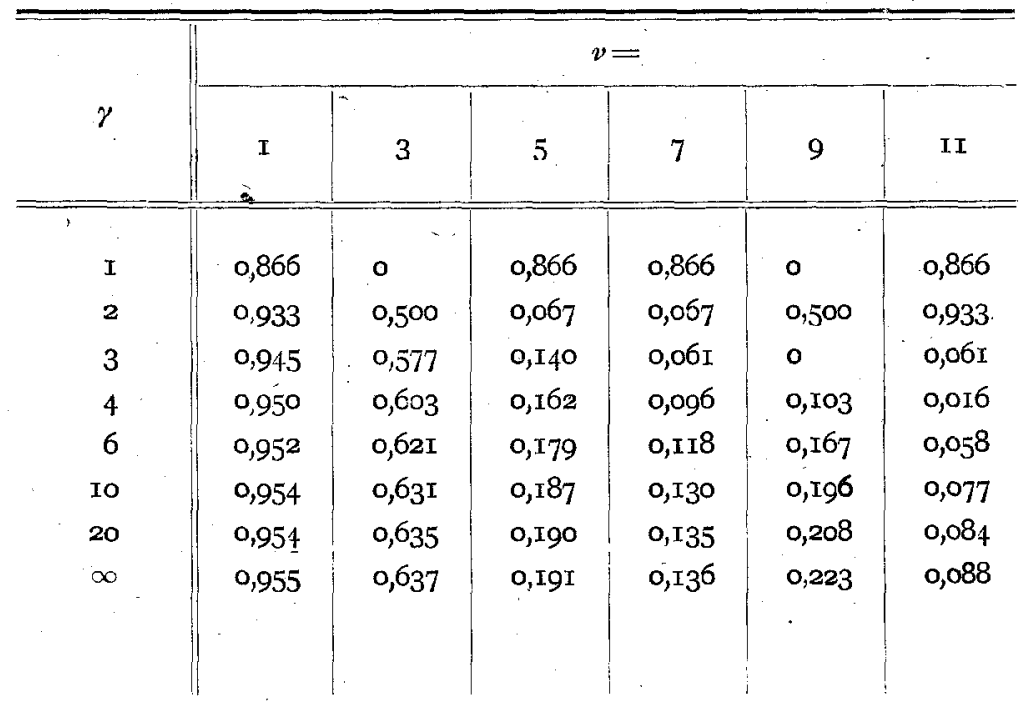

ist also derselbe wie bei den dreiphasigen Bruchlochwicklungen in Abschnitt 4 (vgl. Gl. I5) und kann der Tafel I entnommen werden, wenn darin $\mathrm{q}$ durch $\gamma$ ersetzt wird.

Beide Strahlengruppen sind nun um den Winkel

$$
\varphi=\nu \frac{60^{0}}{\gamma}
$$

in der Phase verschoben, so daß der Wicklungsfaktor des ganzen Wicklungsștranges

$$
\xi_{\nu}=\xi_{\nu}^{\prime} \cdot \xi_{\nu}^{\prime \prime}
$$


wird, worin

$$
\xi_{v}^{\prime \prime}=\frac{\sin \frac{\nu 60^{\circ}}{\gamma}}{2 \sin \frac{\nu 30^{\circ}}{\gamma}} .
$$

Die Faktoren $\xi_{p}^{\prime \prime}$ sind in Tafel 8 zusammengestellt, der wir entnehmen, daß die Wicklungsfaktoren der Bruchlochwicklungen dieses Abschnitts immer kleiner sind, und die Spannungskurve sich weit mehr der Sinusform nähert als bei den Bruchlochwicklungen in Abschnitt 4. Tafel 9 enthält die nach Gl. 2I berechneten vollständigen Wicklungsfaktoren.

Es wäre noch zu untersuchen, wie groß die resultierenden Oberwellen der E.M.K. eines Wicklungsstranges sind, die von der Nutung des Ankers herrühren. Bei den Wicklungen dieses Abschnitts lassen sich die Spannungen der Spulenseiten eines Wicklungsstranges immer in zwei Gruppen $\gamma-\mathrm{I}$ und $\gamma+\mathrm{I}$ Spannungen zerlegen (vgl. Abb. Iob bis I2b und I4b). Die Spannungen jeder Gruppe sind unter sich in der Phase um $\frac{\mathrm{I}}{\mathrm{p}}$ des Phasenwinkels benachbarter Nuten, der für die Oberwellen $\mathrm{n} \stackrel{\text { ter }}{=}$ Art $\mathrm{n} 360^{\circ}$ ist, verschoben. Die Oberwellen, für die $\mathrm{n}<\mathrm{p}$ ist, werden deshalb im wesentlichen unterdrückt, wenn die beiden Gleichungen

$$
\left.\begin{array}{l}
\gamma-\mathrm{I}=\mathrm{gp} \\
\gamma+\mathrm{I}=\mathrm{gp}
\end{array}\right\}
$$

gleichzeitig bestehen, worin $\mathrm{g}$ eine beliebige ganze Zahl ist. Dividieren wir diese Gleichungen durch $\mathrm{p}$, so gehen sie über in

$$
\left.\begin{array}{l}
q=g+\frac{I}{p} \\
q=g-\frac{I}{p} .
\end{array}\right\}
$$

Diese Bedingungen sind für die Polpaarzahl $\mathrm{p}=2$ immer erfüllt, so daß also bei den vierpoligen Wicklungen mit am Ankerumfang gleichmäßig verteilten Nuten die Oberwellen erster Art im wesentlichen unterdrückt werden. Die Oberwellen zweiter Art, die außerdem wohl praktisch nur noch in Frage kommen, werden sich dagegen fast ungeschwächt in der resultierenden Spannung zeigen, weil der Phasenwinkel

$$
\beta_{2}=2 \frac{360^{\circ}}{\mathrm{p}}=360^{\circ}
$$

ist.

Für die übrigen hier in Frage kommenden Polpaarzahlen $(p>2)$ können die GI. 22 nicht gleichzeitig erfüllt werden. Wir erhalten für $\mathrm{n}<\mathrm{p}$ die kleinsten Amplituden der Oberwellen, wenn eine der beiden Gleichungen erfüllt ist, wenn also, wie bei den Wicklungen im Abschnitt 4

$$
\mathrm{q}=\mathrm{g} \mp \frac{\mathrm{I}}{\mathrm{p}}
$$

ist. Die resultierende E.M.K. der Oberwellen ( $\mathrm{n} \stackrel{\text { ter }}{=}$ Art) sämtlicher in Reihe geschalteter Spulenseiten desselben Wicklungsstrangs ist dann nahezu gleich der geometrischen Summe der Oberwellen von nur 2 Spulenseiten, die um den Phasenwinkel $\mathrm{n} \cdot \frac{360^{\circ}}{\mathrm{p}}$ verschoben sind. Die Unterdrückung der Oberwellen ist also noch etwas vollkommener als bei den Wicklungen des Abschnitts 4.

6. Die übrigen symmetrischen Bruchlochwicklungen und die Zahl der parallelen Wicklungszweige. In den Abschnitten 4 und 5 haben wir alle Bruchlochwicklungen mit $t=I$, außerdem auch noch die dreiphasigen Wicklungen mit $t=2$ und $\gamma=$ ungerade behandelt. Diese Bruchlochwicklungen erfüllen die GI. I3 
und sind dadurch gekennzeichnet, daß bei ungerader Spulenzahl im Strang alle Spulen in Reihe geschaitet werden müssen, bei gerader Spulenzahl im Strang aber höchstens zwei gleichwertige Wicklungszweige möglich sind. Die Zahl der möglichen parallelen Wicklungszweige ist also bei den früher behandelten Bruchlochwicklungen dieselbe wie bei einer zweipoligen Wicklung. Für alle.Wicklungen dieser Art sind in den Tafeln 5 bis 7 die Nutenzahlen q für Pol und Strang ohne Klammern gedruckt.

Alle übrigen symmetrischen Bruchlochwicklungen entstehen nun aus den bereits behandelten, indem man Nutenzahl und Polpaarzahl mit demselben Faktor $t^{\prime}$ multipliziert. Es ergeben sich dann immer t'mal so viel gleichwertige Wicklungszweige wie bei den Wicklungen in Abschnitt 4 und 5 , also $t^{\prime}$ oder $2 t^{\prime}$ Wicklungszweige, je nachdem die Spulenzahl im Strang der alten Wicklung ungerade oder gerade ist. Diese Tatsache ergibt sich ohne weiteres daraus, daß der Nutenstern der abgeleiteten Wicklung sich für je $\frac{\mathrm{N}}{\mathrm{t}^{\prime}}$ aufeinander folgende Nuten wiederholt. Wir erhalten die neuen Wicklungen aus den alten, indem wir im abgewickelten Schaltplan je $\mathbf{t}^{\prime}$ alte Wicklungen aneinander reihen, wofür kein Ausführungsbeispiel aufgezeichnet zu werden braucht. Die Nutenzahl q für Pol und Strang ist bei der neuen Wicklung dieselbe wie bei der Wicklung, aus der sie abgeleitet ist. Die Tafeln 5 bis 7 lassen deshalb ohne weiteres erkennen, welche Bruchlochwicklungen abgeleitet sind. Die gleichen Werte von q liegen in den Tafeln auf Strahlen, die von links oben nach rechts unten laufen (vgl. z. B. $q=1 / 2$ in Tafel 7), der Strahl beginnt bei den zugeordneten Werten von $N$ und $p$, für die eine Wicklung mit $\mathbf{t}^{\prime}=\mathrm{I}$ ausführbar ist. Die Werte von $q$ sind für diese Wicklungen, die wir als Urwicklungen bezeichnen können, in den Tafeln 5 bis 7 ohne Klammern gedruckt, die der abgeleiteten Wicklungen dagegen in eckige Klammern gesetzt.

Die Wicklungsfaktoren der Wicklungen dieses Abschnitts sind dieselben wie die Wicklungsfaktoren der entsprechenden Urwicklungen; auch die durch die Nutung des Ankers hervorgerufenen Oberwellen der E.M.K. werden bei allen Wicklungen mit demselben Werte von $\mathrm{q}$ auch in demselben Maße unterdrückt.

Bei einer gegebenen Bruchlochwicklung bestimmt sich die Zahl der möglichen parallelen Wicklungszweige aus dem größten Teiler $t^{\prime}$, durch den Nutenzahl $\mathrm{N}$ und Polpaarzahl p der Bruchlochwicklung derart gekürzt werden können, daß die gekürzte Nutenzahl $\mathrm{N}^{\prime}=\frac{\mathrm{N}}{\mathrm{t}^{\prime}}$ und die gekürzte Polpaarzahl $\mathrm{p}^{\prime}=\frac{\mathrm{p}}{\mathrm{t}^{\prime}}$ wieder die Ausführung einer symmetrischen Bruchlochwicklung ermöglichen. Dies ist nach Gl. I a a der Fall, wenn

$$
\frac{\mathrm{N}^{\prime}}{2 \mathrm{~m}}=\frac{\mathrm{N}}{2 \mathrm{mt}^{\prime}}=\frac{\gamma}{\mathrm{t}^{\prime}}
$$

eine ganze Zahl ist. Die Gl. I2 b und $12 \mathrm{~b}^{\prime}$ sind dann ebenfalls erfüllt.

$$
\frac{\gamma}{\mathbf{t}^{\prime}}=\operatorname{ganz} \text { und } \frac{\mathbf{p}}{\mathbf{t}^{\prime}}=\operatorname{ganz}
$$

sind also die Bedingungen für das Auftreten von mindestens $t^{\prime}$ gleichwertigen Wicklungszweigen.

Für Zweiphasenwicklungen stimmen diese Bedingungen mit Gl. I2 $\mathrm{b}^{\prime}$ überein, denn $t$ in Gl. $12 b^{\prime}$ ist ein Teiler von $p$, nämlich der Teiler, den $N=2 \mathrm{~m} \gamma$ und $\mathrm{p}$ gemeinsam haben. Es ist also für $Z$ weiphasenwicklungen

$$
\mathbf{t}^{\prime}=\mathrm{t}
$$

und die Zahl c der gleichwertigen Wicklungszweige ist

$$
\begin{aligned}
& \text { bei } \frac{\gamma}{\mathrm{t}^{\prime}}=\frac{\gamma}{\mathrm{t}}=\text { gerade: } \quad c=2 \mathrm{t}^{\prime}=2 \mathrm{t} \\
& \text { bei } \frac{\gamma}{\mathrm{t}^{\prime}}=\frac{\gamma}{\mathrm{t}}=\text { ungerade } \quad c \quad c=\mathrm{t}^{\prime}=\mathrm{t} .
\end{aligned}
$$


Wenn bei der Dreiphasenwicklung

$$
\frac{\gamma}{\mathrm{t}}=\operatorname{gan} z
$$

ist, so ist auch für diese Wicklung (Abschnitt 4 )

$$
\mathfrak{t}^{\prime}=\mathrm{t}
$$

und die Zahl der' gleichwertigen Wicklungszweige ist

$$
\begin{aligned}
& \text { bei } \frac{\gamma}{\mathrm{t}^{\prime}}=\frac{\gamma}{\mathrm{t}}=\text { gerade: } \quad \mathrm{c}=2 \mathrm{t}^{\prime}=2 \mathrm{t} \\
& \text { bei } \frac{\gamma}{\mathrm{t}^{\prime}}=\frac{\gamma}{\mathrm{t}}=\text { ungerade: } \mathrm{c}=\mathrm{t}^{\prime}=\mathrm{t} .
\end{aligned}
$$

Nach Gl. I2 b kann aber $\frac{\gamma}{t}$ auch ein Bruch mit dem Nenner 2 sein (Abschnitt 5),

$$
\frac{\gamma}{\mathrm{t}}=\text { gebrochen. }
$$

Es ist dann

$$
\mathrm{t}^{\prime}=\frac{\mathrm{t}}{2}
$$

und die Zahl der möglichen parallelen Wicklungszweige, da $\gamma$ in diesem Falle immer ungerade ist,

$$
\mathrm{c}=\mathrm{t}^{\prime}=\frac{\mathrm{t}}{2} \text {. }
$$

Diese Zahlen der parallelen Wicklungszweige der Bruchlochwicklungen lassen sich aus den Tafeln 3 und 4 ablesen, sie sind aber der Übersichtlichkeit wegen in den Tafeln Io und I I besonders zusammengestellt.

Die Zahl der möglichen parallelen Wicklungszweige können wir auch den Tafeln 5 bis 7 entnehmen, welche die Zahlen q für Pol und Strang enthalten. Es ist

$$
q=\frac{N}{2 p m}=\frac{\gamma}{p} \text {. }
$$

Wenn, wie in den Tafeln 5 bis $7 \mathrm{q}$ als gebrochene Zahl in der Form

$$
q=g+\frac{z}{n}
$$

geschrieben wird, so daß $z$ und $n$ keinen gemeinsamen Teiler mehr haben, $\gamma$ und $\mathrm{p}$ also durch den größten gemeinsamen Teiler $\mathrm{t}^{\prime}$ gekürzt sind, so ist

$$
\mathbf{t}^{\prime}=\frac{\mathbf{p}}{\mathbf{n}}
$$

Das Verhältnis aus der Polpaarzahl und dem kleinsten Nenner $\mathrm{n}$ des Bruches q gibt also die volle oder die halbe Zahl der möglichen parallelen Wicklungszweige an, je nachdem das Verhältnis $\frac{\gamma}{t^{\prime}}$ gerade oder ungerade ist.

Wenn $\mathrm{n}=\mathrm{p}$ ist,- sind bei geraden Zahlen von $\gamma$ (am Kopfe der Tafeln) zwei, bei ungeraden Zahlen von $\gamma$ gar keine parallelen Wicklungszweige möglich.

Wenn $\frac{\mathrm{p}}{\mathrm{n}}=\mathrm{t}^{\prime}$ ist, sind mit $\frac{\gamma}{\mathrm{t}^{\prime}}=$ gerade $2 \mathrm{t}^{\prime}$, mit $\frac{\gamma}{\mathrm{t}^{\prime}}=$ ungerade $\mathrm{t}^{\prime}$ parallele Wicklungszweige möglich. So finden wir $z$. B. in Tafel 7 bei $p=I 0$ und $\gamma=16, q=1^{3 / 5}$; es ist also $\mathrm{t}^{\prime}=\frac{\mathrm{p}}{\mathrm{n}}=\frac{\mathrm{IO}}{5}=2$. Da $\frac{\gamma}{\mathrm{t}^{\prime}}=\frac{\mathrm{I} 6}{2}=8$ eine gerade Zahl, so ist die Zahl der möglichen parallelen Wicklungszweige $2 t^{\prime}=4$. Ferner ist in Tafel 6 bei $p=I 4$ und $\gamma=7, \mathrm{q}=1 / 2$. Es ist $\mathrm{t}^{\prime}=\frac{\mathrm{p}}{\mathrm{n}}=\frac{\mathrm{I} 4}{2}=7$ und $\frac{\gamma}{\mathrm{t}^{\prime}}=\frac{7}{7}=\mathrm{I}$ eine ungerade Zahl. Die Zahl der möglichen parallelen Wicklungszweige ist also $\mathfrak{t}^{\prime}=7$. 


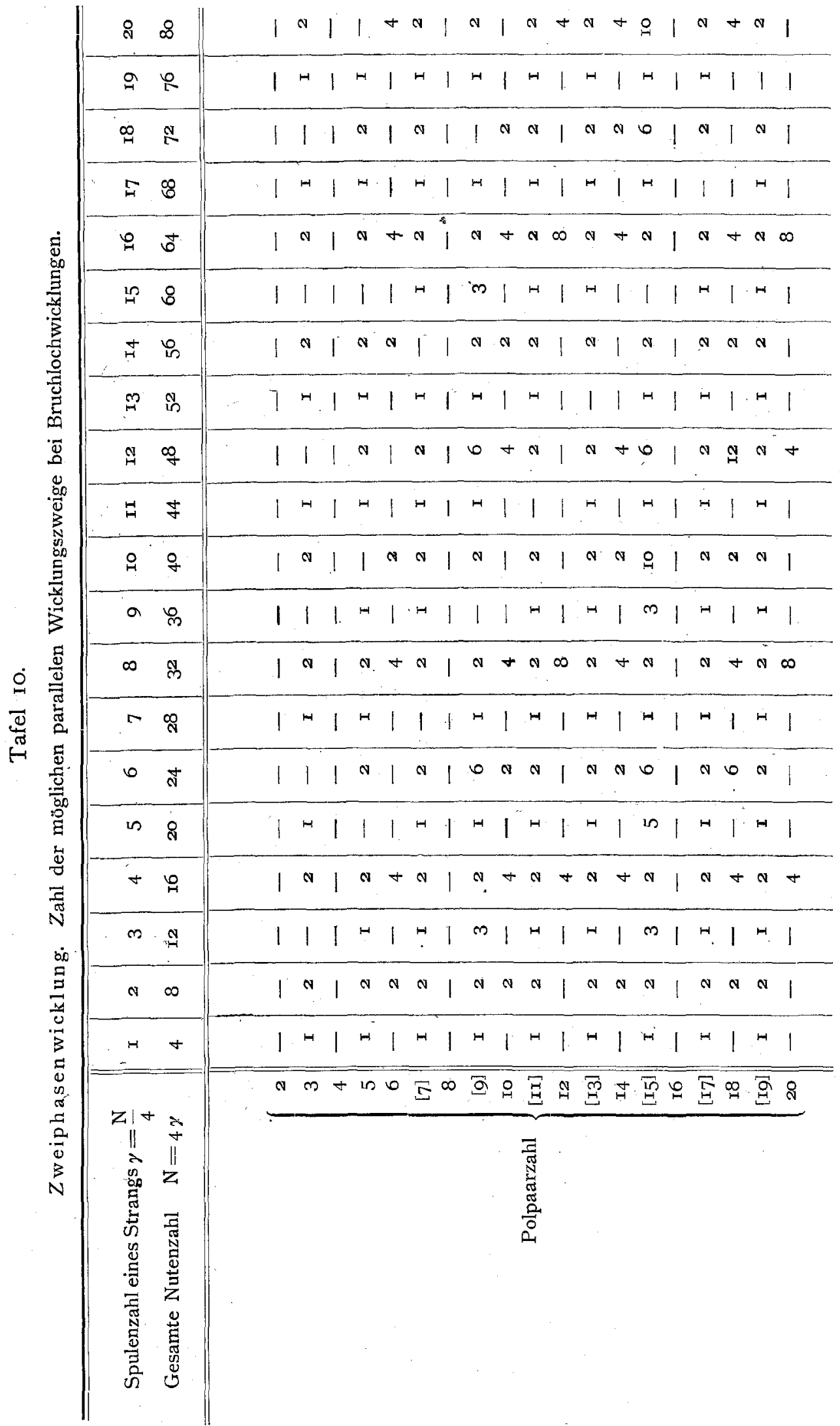




\begin{tabular}{|c|c|c|c|c|c|c|c|c|c|c|c|c|c|c|c|}
\hline & ㅇำ & $\underset{i}{\stackrel{8}{*}}$ & 11 & 1 & 11 & $N$ & +1 & 10 & a 1 & $N$ & +1 & $\dot{t}$ & ล & $n$ & 1 \\
\hline & $\stackrel{2}{\circ}$ & $\underset{H}{\stackrel{D}{H}}$ & -1 & -1 & -1 & $H$ & H I & H & $H 1$ & $r$ & -1 & H & $H .1$ & 1 & $H$ \\
\hline & $\stackrel{\infty}{\stackrel{\infty}{H}}$ & $\underset{⿴ 囗 十)}{\infty}$ & 11 & N & N I & N & N 1 & N & $\curvearrowright 0$ & a & N 0 & a & a & ง & N \\
\hline & $\mathbb{E}$ & 议 & -1 & $m$ & $M \mid$ & $H$ & -1 & $\rightarrow$ & -1 & $H$ & $H$ & $m$ & 11 & $m$ & $H$ \\
\hline 竎 & $\stackrel{0}{\circ}$ & $\%$ & 11 & 1 & o I & a & 11 & + & N I I & N & +1 & 1 & s , । & $\mathrm{N}$ & $\infty$ \\
\hline$\frac{3}{3}$ & $\mathscr{R}$ & 8 & $\mapsto \quad \mid$ & $H$ & $1 m$ & $H$ & $H \cdot \mid$ & in & H m & $H$ & 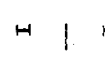 & $H$ & -1 & $H$ & in \\
\hline 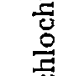 & 苾 & $\infty$ & 11 & $\mathrm{~N}$ & $\alpha^{-1}$ & 1 & N & $\mathrm{N}$ & N 1 & * & 11 & $N$ & $\mathrm{~N}$ & $w$ & $\alpha$ \\
\hline 总 & 9 & $\stackrel{\infty}{2}$ & $m 1$ & $H$ & $n-1$ & $H$ & $H \quad 1$ & $H$ & -1 & 1 & $H \quad 1$ & $H$ & +1 & 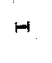 & $H$ \\
\hline$\underset{D}{0}$ & 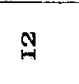 & $\stackrel{n}{N}$ & 11 & 1 & $N \mid$ & N & +1 & $\forall$ & N 1 & $N$ & +0 & $\theta$ & * 1 & $\mathrm{~N}$ & $\forall$ \\
\hline $\begin{array}{l}3 \\
N \\
n \\
0 \\
00\end{array}$ & $\stackrel{H-1}{-1}$ & 8 & $m 1$ & $H$ & $H \quad 1$ & $H$ & -1 & $H$ & 11 & - & -1 & $m$ & $w 1$ & H & $H$ \\
\hline$\frac{E}{3}$ & 욤 & 8 & 11 & $N$ & 11 & N & * 1 & 1 & N 1 & o & $\times \quad$ & $N$ & a 1 . & o & 욤 \\
\hline$\frac{3}{5}$ & $a$ & in. & -1 & $H$. & $H$ & $H$ & -11 & ' & $H \quad m$ & $H$ & $H m$ & $m$ & $H a$ & $H$ & $H$ \\
\hline 氶 & $\infty$ & $\stackrel{\infty}{\forall}$ & 1.1 & 1 & N & $\mathrm{N}$ & $1 \cdots 1$ & $\nabla$ & N 1 & $N$ & +10 & $\infty$ & * । & $\mathbf{N}$ & $\infty$ \\
\hline ت્ّ & $\sigma$ & $\stackrel{N}{+}$ & -1 & -1 & $H \quad 1$ & 1 & -1 & +1 & $\because i$ & $H$ & $N 1$ & $H$ & -1 & $m$ & $H$ \\
\hline $\begin{array}{l}:= \\
: 0 \\
: 0\end{array}$ & 0 & 卢 & 11 & N & ल । & N & N I & $\mathrm{N}$ & $N 0$ & $N$ & 06 & N & N । & $\dot{\alpha}$ & N \\
\hline 矛 & in & 品 & -1 & $H$ & 11 & $H$ & m I & in & $m$ I & $H$ & $H \mid$ & $m$ & $\mapsto \quad \mid$ & $H$ & Ln \\
\hline$\frac{E}{N}$ & + & ते & 11 & 1 & $\times 1$ & N & +1 & + & o 1 & $N$ & +1 & + & N 1 & N & + \\
\hline $\begin{array}{l}\infty \\
\Xi \\
\Xi\end{array}$ & $m$ & $\stackrel{\infty}{\infty}$ & -1 & $M$ & $H m$ & $H$ & +1 & $H$ & $H \quad n$ & $H$ & $H n$ & $H$ & +1 & $H$ & $m$. \\
\hline $\overrightarrow{\overrightarrow{3}}$ & $\mathrm{~N}$ & $\stackrel{N}{\stackrel{N}{*}}$ & 11 & $\mathrm{~N}$ & N 1 & a & N I & 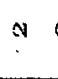 & N 1 & N & o I & N & $N$ & N & $N$ \\
\hline $\begin{array}{l}0 \\
0 \\
0 \\
n \\
\pi \\
0\end{array}$ & $-H$ & 0 & $\rightarrow 1$ & $H$ & +1 & $H$ & $\rightarrow \quad 1$ & H & $m 1$ & $H$ & $H \quad \mid$ & $m$ & $+i \mid$ & $H$ & $H$ \\
\hline$\frac{a}{2}$ & $z / 0$ & & $\alpha B$ & + & in 0 & $E$ & $\infty$ as & 只 & 帠 & $\stackrel{m}{*}$ & $\underset{H}{\mathbb{W}}$ & $\stackrel{0}{\circ}$ & 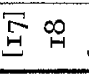 & $\underset{9}{9}$ & ㅇ. \\
\hline$\stackrel{2}{a}$ & 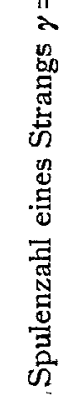 & 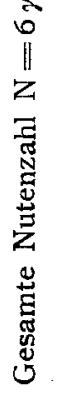 & & & & & & $\frac{3}{0}$ & 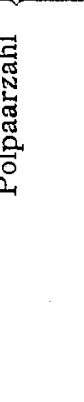 & & & & & & . \\
\hline
\end{tabular}


Zur Beurteilung des Wicklungsfaktors und der von der Nutung des Ankers herrührenden Oberwellen dient der Nenner $\mathrm{n}$ in den Zahlen von $\mathrm{q}$ der Tafeln 5 bis 7 . Je nachdem $\mathrm{n}$ ungerade oder gerade ist; gelten für den Wicklungsfaktor und die Unterdrückung der Oberwellen die in den Abschnitten 4 oder 5 gefundenen Ergebnisse.

\section{B. Bruchlochwicklungen mit mehreren unbewickelten Nuten.}

7. Die Ausführbarkeit. Aus jeder Ganzlochwicklung entsteht eine Bruchlochwicklung, wenn die Nuten der Ganzlochwicklung um eine unbewickelte Nut vermehrt, und die so erhaltenen Nuten gleichmäßig am Ankerumfang verteilt werden. Damit die mehrphasigen Bruchlochwicklungen symmetrisch werden, müssen meh rere Nuten unbewickelt bleiben. Andererseits ist es nicht wie bei der Ganzlochwicklung notwendig, daß die Spulenzahl im Strang durch die Polpaarzahl teilbar ist. Wir wollen zunächst die Bedingungen aufstellen, denen die Nutenzahl einer symmetrischen Bruchlochwicklung mit unbewickelten Nuten und beliebiger Spulenzahl im Strang genügen muß.

Bei der Ein phas en wicklung ist nur ein einziger Wicklungsstrang vorhanden; deshalb ist der Begriff der Symmetrie hier eigentlich bedeutungslos. Wenden wir aber die Definition der Symmetrie von Mehrphasenwicklungen (vgl. Abschnitt 3) sinngemäß auf die Einphasenwicklung $(\mathrm{m}=\mathrm{I})$ an, so ergibt sich, daß jede Einphasenwicklung symmetrisch ist.

Für Dreileiternetze werden Einphasenwicklungen mit zwei Wicklungsabteilungen ausgeführt, deren Spannungen gleiche Größe haben und in der Phase um $180^{0}$ verschob̄en sind; es sind dies im eigentlichen Sinne ,Zweiphasenwicklungen“. Wir brauchen diese Wicklungen nicht besonders zu behandeln, weil sie sich aus jeder Einphasenwicklung mit einer geraden Zahl gleichwertiger Wicklungszweige durch entsprechende Schaltung der Zweige ergeben.

Bezeichnen wir mit $\eta$ die Zahl der unbewickelten Nuten und mit $\gamma$ wieder die Zahl der Spulen im Strang - bei den Einphasenwicklungen also die gesamte Spulenzahl - so wird die Nutenzahl der einphasigen Bruchlochwicklung

$$
\mathrm{N}=2 \gamma+\eta
$$

wobei $\eta$ im allgemeinen aber nicht willkürlich gewählt werden darf.

Für die Ausführbarkeit einer Bruchlochwicklung genügt es, wenn entweder die (bewickelte) Nutenzahi für Pol und Strang

$$
\mathrm{q}=\frac{\gamma}{\mathrm{p}}
$$

oder das Verhältnis von Nutenzahl und Produkt aus Polzahl und Strangzahl

$$
Q=\frac{\mathrm{N}}{2 \mathrm{pm}}
$$

ein Bruch ist (vgl. Abschnitt I). Wenn daher

$$
\mathrm{q}=\frac{\boldsymbol{\gamma}}{\mathrm{p}}=\text { gebrochen }
$$

ist, kann die Zahl der unbewickelten Nuten nach Gl. 27

sein. Ist dagegen

$$
\eta=\text { beliebig }
$$

$$
\mathrm{q}=\frac{\boldsymbol{\gamma}}{\mathrm{p}}=\operatorname{gan} \mathrm{z}
$$

so muß

$$
\frac{\eta}{2 \mathrm{p}}=\text { gebrochen }
$$

sein, damit bei der Einphasenwicklung $(\mathrm{m}=\mathrm{I})$

gebrochen wird.

$$
\mathrm{Q}=\frac{\mathrm{N}}{2 \mathrm{p}}=\mathrm{q}+\frac{\eta}{2 \mathrm{p}}
$$


Bei passender Wahl der. Spulenzahl läßt sich für jede Nuten- und Polpaarzahl eine einphasige Bruchlochwicklung mit mehreren unbewickelten Nuten ausführen.

Die nach dem heutigen Sprachgebrauch als $Z$ weiphasenwicklung bezeichnete Wicklung verlangt, wie wir in Abschnitt 3 gesehen haben, daß die Zahl der ungleichphasigen Nuten durch 4 teilbar ist, damit die Wicklung symmetrisch wird. Wenn wir mit $t$ wieder den Teiler bezeichnen, den die Nutenzahl $N$ und die Polpaarzahl p gemeinșam haben, so ist $\frac{\mathrm{N}}{\mathrm{t}}$ die Zahl der ungleichphasigen Nuten. Schreiben wir für die Nutenzahl der Zweiphasenwicklung

$$
\mathrm{N}=4 \gamma+\eta
$$

so muß also für die symmetrische Wicklung

$$
\frac{N}{4 t}=\frac{4 \gamma+\eta}{4 t}=g
$$

sein, worin $\mathrm{g}$ eine ganze Zahl ist. Die Zahl der unbewickelten Nuten ergibt sich hieraus $z u$

$$
\eta=4(\operatorname{tg}-\gamma)
$$

Da $\mathrm{tg}$ und $\gamma$ immer ganze Zahlen sind, so ist auch $(\mathrm{tg}-\gamma)$ eine ganze Zahl. Die Zahl der unbewickelten Nuten muß also immer durch 4 teilbar sein. Daraus folgt ohne weiteres, daß mit derselben Nutenzahl auch eine symmetrische Zweiphasenwicklung ausgeführt werden kann, bei der alle Nuten bewickelt sind, und zwar eine Bruchlochwicklung, wenn das Verhältnïs Q.gebrochen ist. Die Zweiphàsenwicklungen mit mehreren unbewickelten Nuten haben deshalb im allgemeinen keine. praktische Bedeutung, weshalb wir sie nicht weiter verfolgen wollen.

Bei den symmetrischen Dreiphasenwicklungen muß nach unsern Überlegungen in Abschnitt 3 die Zahl der bewickelten Nuten durch 6 und die Gesamtzahl der ungleichphasigen Nuten $\left(\frac{N}{t}\right)$ durch 3 teilbar sein. Wir müssen also für die Zahl der gesamten Nuten der dreiphasigen Bruchlochwicklung mit mehreren unbewickelten Nuten

$$
\mathrm{N}=6 \gamma+\eta
$$

schreiben, wobei die Zahl $\eta$ der unbewickelten Nuten durch die Gleichung

$$
\frac{\mathrm{N}}{3 \mathrm{t}}=\frac{6 \gamma+\eta}{3 \mathrm{t}}=\text { ganz }
$$

bestimmt ist. Diese Bedingung läßt sich nur erfüllen, wenn $\eta$ mindestens durch 3 teilbar ist. Beschränken wir uns auf den praktisch wichtigsten Fall, wo $\eta$ gleich der kleinsten möglichen Zahl, nämlich

ist, so wird die Nutenzahl

$$
\eta=3
$$

und nach Gl. $29 \mathrm{~b}$ muß

$$
\mathrm{N}=6 \gamma+3
$$

$$
\frac{2 \gamma+\mathrm{I}}{\mathrm{t}}=\operatorname{gan} z
$$

sein. Da der Zähler $(2 \gamma+\mathrm{I})$ in Gl. $30 \mathrm{~b}$ immer ungerade ist, so ist Gl. $30 \mathrm{~b}$ erfüllt, wenn die Spulenzahl im Strang

$$
\gamma=\mathbf{g t}+\frac{\mathbf{t}-\mathbf{I}}{\mathbf{2}}
$$

$(30 \mathrm{C}$

ist, worin $g$ eine beliebige ganze Zahl (einschließlich null). zahl m

Das Verhältnis von Nutenzahl N und Produkt aus Polpaarzahl $p$ und Strang-

$$
\mathrm{Q}=\frac{\mathrm{N}}{2 \mathrm{pm}}
$$

ist in Tafel 12 für alle symmetrischen Wicklungen mit 3 unbewickelten Nuten ein- 


\begin{tabular}{|c|c|c|}
\hline & & 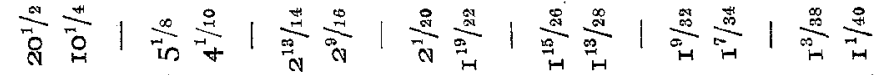 \\
\hline$\stackrel{9}{9}$ & 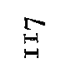 & 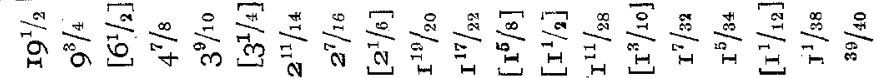 \\
\hline$\stackrel{\infty}{\infty}$ & $\stackrel{G}{G}$ & 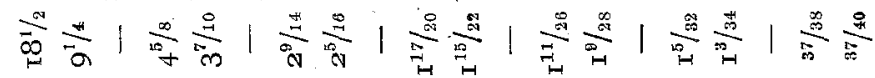 \\
\hline$\approx$ & 8 & 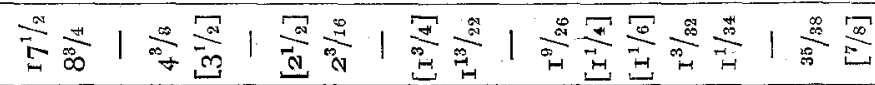 \\
\hline$\stackrel{D}{H}_{1}$ & \& & के \\
\hline $\mathbb{R}$ & 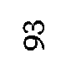 & 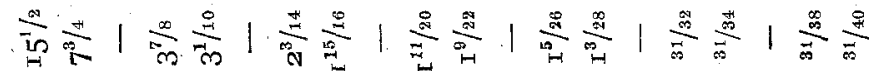 \\
\hline 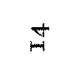 & $\infty$ & 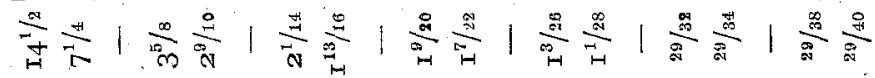 \\
\hline$\stackrel{m}{m}$ & $\vec{\infty}$ & 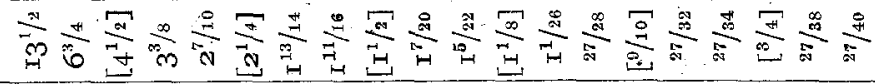 \\
\hline I & 12 & 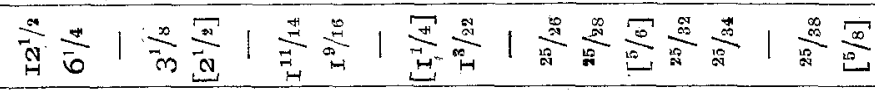 \\
\hline$\stackrel{H}{=}$ & 8 & 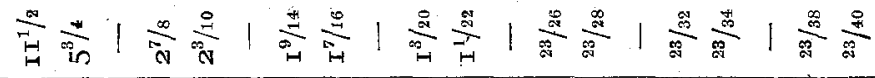 \\
\hline$\stackrel{\circ}{9}$ & 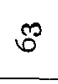 & 事 的空 \\
\hline$a$ & in & क क \\
\hline$\infty$ & है & 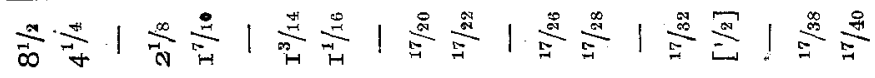 \\
\hline r & $q$ & 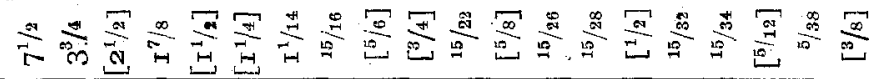 \\
\hline 0 & 今े & 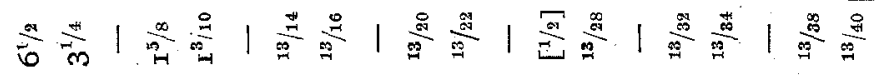 \\
\hline in & $\ddot{m}$ & in \\
\hline t & $\widehat{\kappa}$ & 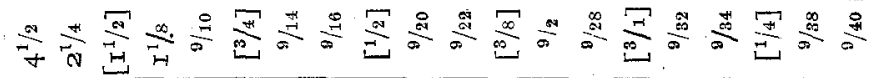 \\
\hline$m$ & है- & 䑻 \\
\hline N & $\stackrel{B H}{H}$ & 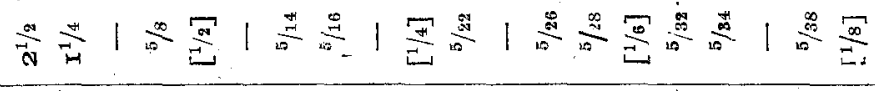 \\
\hline$H$ & $a$ & 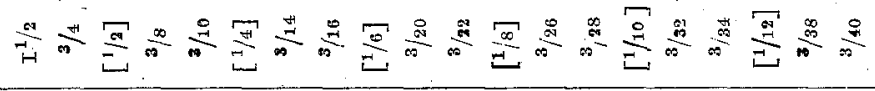 \\
\hline 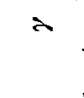 & & $N$ \\
\hline
\end{tabular}

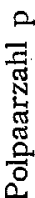


geschrieben. Für die Wicklungen, deren Nutenzahl $N$ und Polpaarzahl p keinen gemeinsamen Teiler besitzen $(t=I)$, alle Nuten also verschiedene Phase haben, sind zum Unterschied von den übrigen die Zahlen $Q$ ohne Klammern gedruckt. Wir er. halten hier auch bei zweipoligen Maschinen $(\mathrm{p}=\mathrm{I})$ für $\mathrm{Q}$ eine gebrochene Zahl.

Aus den Urwicklungen der Tafel I2 ergeben sich neue Wicklungen mit demselben Verhältnis $Q$, wenn wir Nutenzahl und Polpaarzahl mit dem Faktor $t^{\prime}$ multiplizieren; die Zahl der unbewickelten Nuten ist dann $\eta=3 \mathrm{t}^{\prime}$. Diese Wicklungen sind in der Tafel I2, die nur für Wicklungen mit 3 unbewickelten Nuten gilt, nicht enthalten, da sie auch mit andern Nutenzahlen ausführbar sind, als sie am.Kopf der Tafel angegeben sind.

Wir wollen nun an einigen Beispielen den Entwurf der Wicklungen und ihre Eigenschaften näher erläutern.

8. Einphasige Bruchlochwicklungen. Am praktisch wichtigsten sind die Bruchlochwicklungen, bei denen die Nutenzahl $\mathrm{N}$ und die Polpaarzahl $\mathrm{p}$ keinen gemeinsamen Teiler besitzen. Alle Nuten haben dann verschiedene Phase. Wir ererhalten also die größte Zahl der ungleichphasigen Nuten.

Wir wollen zunächst eine Bruchlochwicklung für $\mathrm{N}=27$ Nuten und $\mathrm{p}=4$ Polpaare entwerfen. Der Nutenstern (Abb. I6a) erhält 27 ungleichphasige Strahlen. Wenn die Nuten des Ankers fortlaufend numeriert werden, bezeichnen die an die Strahlen in Abb. I6a angeschriebenen Zahlen die entsprechenden Nuten. Benachbarte Nuten (z. B. $I$ und 2) liegen in der Phase um $\mathrm{p}=4$ Strahlen auseinander. Im vorliegenden Falle können wir, wie bei den üblichen einphasigen Ganzlochwicklungen genau $2 / 3$ aller Nuten bewickeln. Es wird dann

$$
\gamma=\eta=\frac{\mathrm{N}}{3}=9
$$

und die Nutenzahl für Pol und Strang ist

$$
\mathrm{q}=\frac{\gamma}{\mathrm{p}}=2^{1} / 4
$$

$\mathrm{q}$ ist also gebrochen, so daß nach Gl. 27 a $\eta$ beliebig, also $\gamma=\eta=9, Q=3^{3 / s,} q=2^{1 / 4}$. auch gleich $\gamma$ gewählt werden $\operatorname{darf}^{1}$ ).

Um die Wicklung möglichst gut auszunützen, müssen die Spannungen der gleichsinnigen Spulenseiten möglichst wenig Phasenunterschied aufweisen, und die beiden Resultierenden aus den Spannungen der positiven und der negativen Spulen-

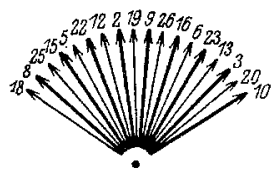

Abb. 16b. Einzelspannungen für die Wicklung zu Abb. I6a.

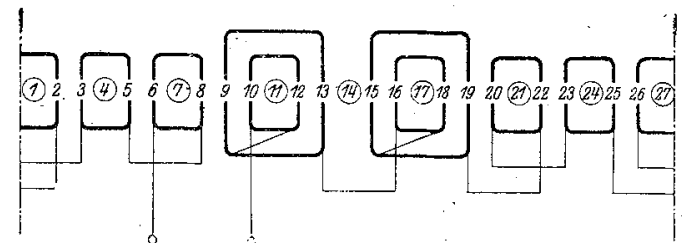

Abb. 16c. Schaltplan einer einphasigen Bruchlochwicklung nach $\mathrm{Abb}$. I6a und $\mathrm{b}$. Reihenschaltung der Spulen.

seiten jedes Stranges müssen möglichst um $180^{\circ}$ in der Phase verschoben sein. Wenn wir dies beachten, sind die Spulenseiten so auf die Nuten des Ankers zu verteilen, wie es durch den Spannungsstern in Abb. 16a veranschaulicht wird. Die Nuten, in

^) Wenn. ${ }^{2} / 3$ aller Nuten bewickelt sein sollen, muß die gesamte Nutenzahl

$$
\mathrm{N}=3 \gamma
$$

sein. Hierfür ist eine Bruchlochwicklung ausführbar, wenn entweder

$$
\mathrm{q}=\frac{\gamma}{\mathrm{p}}=\text { gebrochen oder } \mathrm{Q}=\frac{3}{2} \mathrm{q}=\text { gebrochen ist. }
$$


denen positive Spulenseiten liegen, sind durch starke, die, in denen negative Spulenseiten liegen, durch schwache voll ausgezogene Linien angedeutet; die strichpunktierten Linien mit den kleinen Kreisen entsprechen den unbewickelten Nuten. Die Phase der Einzelspannungen wird nach Herstellung der Spulenquerverbindungen durch $\mathrm{Abb}$. I6b veranschaulicht. In $\mathrm{Abb}$, I6c ist die Wicklung im abgewickelten Schaltplan dargestellt. Sämtliche Spulen müssen in Reihe geschaltet werden, weil sich die Einzelspannungen der Wicklung nicht in zwei Gruppen zerlegen lassen, deren Resultierende nach Größe und Phase gleich sind.

Als Beispiel für eine Wicklung mit ganzer Nutenzahl $q=3$ für Pol und Strang wählen wir $\mathrm{p}=3$ und $\gamma=9$. Die Zahl der unbewickelten Nuten $\eta$ muß dann nach Gl. 27 b so gewählt werden, daß sie nicht durch die Polzahl teilbar ist. Damit N und $\mathrm{p}$ teilerfremd und annähernd $2 / 3$ aller Nuten bewickelt sind, wählen wir $\eta=8$; die Nutenzahl des Ankers wird dann

$$
\mathrm{N}=2 \gamma+\eta=26
$$

In $\mathrm{Abb} . \mathrm{I} 7 \mathrm{a}$ ist der Nutenstern und in $\mathrm{Abb}$. I $7 \mathrm{~b}$ sind die Einzelspannungen dieser

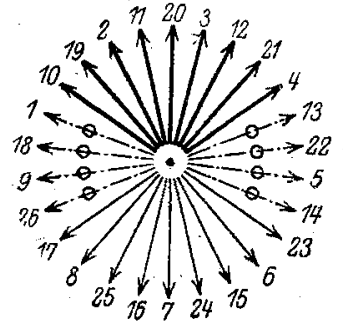

Abb. I7 a. Nutenstern einer einphasigen Bruchlochwicklung mit $\mathrm{N}=26$, $\mathrm{p}=3, \quad \gamma=9, \quad \eta=8$, $Q=4^{1 / s,} \quad q=3$. Wicklung dargestellt. Der Schaltplan wird durch Abb. I7 c veranschaulicht. Auch in diesem Falle sind keine parallelen Wicklungszweige möglich, weil $\gamma$ ungerade ist.

Durch Vergleich der Nutensterne in Abb. I6a und I7a übersehen wir leicht, daß bei Wicklungen mit $\mathrm{N}$ und $\mathrm{p}$ teilerfremd nur dann parallele Wicklungszweige möglich sind, wenn sowohl die Nutenzahl $\mathrm{N}$ als auch die Spulenzahl $\gamma$ gerade ist,

$$
\mathrm{N}=\text { gerade und } \gamma=\text { gerade }
$$

Nur in diesem Falle lassen sich die Spulenseiten in je zwei Gruppen, enthaltend gleich viele positive und negative Spulenseiten, zusammenfassen, deren resultierende Spannungen nach Größe und Phase gleich sind. Wir wählen als Beispiel eine sechspolige $(p=3)$ Wicklung mit

$$
\mathrm{N}=\mathbf{2 2}=\text { gerade und } \gamma=8=\text { gerade. }
$$

Die Zahl der unbewickelten Nuten muß dann nach Gl. $27 \quad \eta=6$ sein. Der Nuteñstern für diese Wicklung ist in $\mathrm{Abb}$. $18 \mathrm{a}$, die Einzelspannungen in $\mathrm{Abb}$. I8b

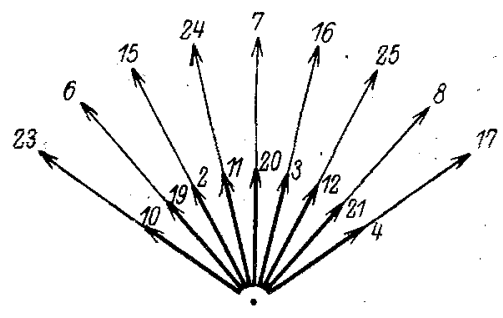

Abb. I7b. Einzelspannungen für die Wicklung zu Abb. I7a.

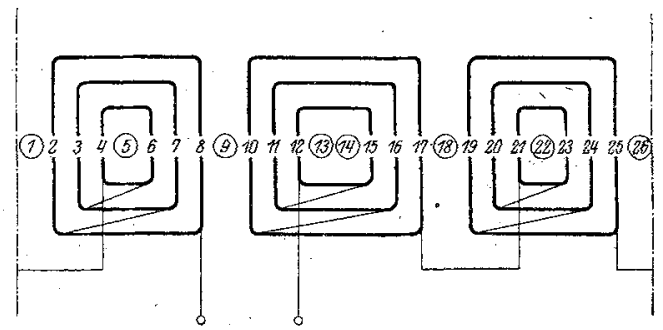

Abb. I7c. Schaltplan einer einphasigen Bruchlochwicklung nach Abb. I7 a und b. Reihenschaltung der Spulen.

und der abgewickelte Schaltplan in Abb. I8c dargestellt, wobei die beiden gleichwertigen Wicklungszweige parallel geschaltet sind.

Eine Einphasenbruchlochwicklung läßt sich auch für die Polpaarzahl $\mathrm{p}=\mathrm{I}$ ausführen. $\frac{\gamma}{\mathrm{p}}$ ist dann immer eine ganze Zahl, das Verhältnis $Q$ von Nụtenzahl $N$ und Polzahl $2 \mathrm{p}=2$ muß deshalb gebrochen sein, was für ungerade Nutenzahlen immer der Fall ist.

Aus den Bruchlochwicklungen, deren Nutenzahi $N$ und Polpaarzahl p teiler- 
fremd sind, lassen sich andere Bruchlochwicklungen ableiten, indem Nutenzahl $N$ und Polpaarzahl $p$ der alten Wicklung mit einer beliebigen ganzen Zahl $\mathfrak{t}^{\prime}$ multipliziert werden. Die Zahl der möglichen parallelen Wicklungszweige ist dann $\mathrm{c}=\mathrm{t}^{\prime}$, wenn die Nutenzahl der alten Wicklung ungerade ist. Die Zahl der möglichen parallelen Wicklungszweige ist $\mathrm{c}=2 \mathrm{t}^{\prime}$, wenn sowohl die Nutenzahlen $\mathrm{N}$ als auch die Spulenzahl $\gamma$ der alten Wicklung eine gerade Zahl ist. Auf die übrigen für Einphasenstrom möglichen Bruchlochwicklungen wollen wir hier nicht näher eingehen, da sie weniger wichtig sind.

Die einphasigen Bruchlochwicklungen sind auch für die mit Gleichstrom gespeisten Wicklungen von zylindrischen Feldmagneten geeignet. Es können damit den Wechselstrom-Ganzlochwicklungen solcher Maschinen die Eigenschaften von Bruchlochwicklungen verliehen und die Schwankungen des Induktionsflusses bei passender Nutenzahl im Feldmagneten verhindert oder verringert werden.

Der Wicklungsfaktor der ten. Welle einer Ein-

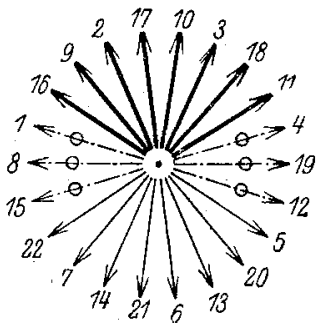

Abb. 18 a. Nutenstern einer einphasigen Bruchlochwicklung mit $\mathrm{N}=22$, $\mathrm{p}=3, \quad y=8, \eta=6$, $Q=3^{2} / 3, q=2^{2} / 3$. phasen-Ganzlochwicklung ist nach Gl. 8 mit

$$
\begin{gathered}
\mathrm{s}=\mathrm{q} \text { und } \varphi=\nu \frac{\mathrm{p}}{\mathrm{N}} 360^{\circ}=\nu \frac{180^{\circ}}{\mathrm{Q}} \\
\xi_{\nu}=\frac{\sin \nu \mathrm{q} \frac{90^{\circ}}{\mathrm{Q}}}{\mathrm{q} \sin \nu \frac{90^{\circ}}{\mathrm{Q}^{-}}} .
\end{gathered}
$$

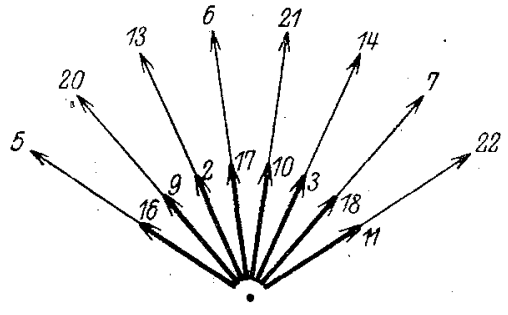

Abb. I8b. Einzelspannungen für die Wicklung zu Abb. 18a.

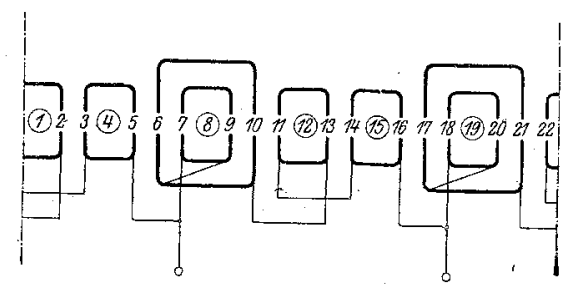

Abb. I8c. Schaltplan einer einphasigen Bruchlochwicklung nach Abb. I8 a u. b. Parallelschaltung der beiden Wicklungszweige.

Bei den einphasigen Bruchlochwicklungen, deren Nutenzahl und Polpaarzahl teilerfremd sind, ist bei gerader Nutenzahl N (vgl. Abb. i $7 \mathrm{~b}$ und $18 \mathrm{~b}$ )

und der Wicklungsfaktor

$$
\mathrm{s}=\gamma \text { und } \varphi=\boldsymbol{\nu} \frac{360^{\circ}}{\mathrm{N}}
$$

$$
\xi_{v}=\frac{\sin \nu \gamma \frac{\mathrm{I} 80^{0}}{\mathrm{~N}}}{\gamma \sin v \frac{\mathrm{I} 80^{0}}{\mathrm{~N}}}
$$

Bei ungerader Nutenzahl (vgl. Abb. 16b) sind die Spannungsphasen der negativen Spulenseiten je um $\frac{360^{\circ}}{2, \mathrm{~N}}$ gegenüber denen der positiven Spulenseiten verschoben. In G1. 8 ist deshalb

$$
\mathrm{s}=2 \gamma \text { und } \varphi=v \frac{360^{\circ}}{2 \mathrm{~N}}
$$


zu setzen. Der Wicklungsfaktor wird in diesem Falle

$$
\xi_{\nu}=\frac{\sin 2 \gamma \nu \frac{180^{\circ}}{2 \mathrm{~N}}}{2 \gamma \sin \nu \frac{180^{0}}{2 \mathrm{~N}}},
$$

wofür wir auch schreiben können

$$
\xi_{v}=\cos \nu \frac{90^{\circ}}{\mathrm{N}} \cdot \frac{\sin \nu \gamma \frac{\mathrm{I} 80^{\circ}}{\mathrm{N}}}{\gamma \sin \nu \frac{\mathrm{I} 80^{0}}{\mathrm{~N}}} .
$$

In Tafel 13 sind die Wicklungsfaktoren (Gl. 32) der Ganzlochwicklungen mit

$$
\frac{\mathrm{q}}{\mathrm{Q}}=\frac{2}{3}
$$

für verschiedene Werte von $Q$ bis zur II. Welle zusammengestellt. Dieser Tafel können auch die Wicklungsfaktoren der Einphasen-Bruchlochwicklungen mit gerader Nutenzahl und

$$
\frac{2 \gamma}{\mathrm{N}}=\frac{\mathrm{q}}{\mathrm{Q}}=\frac{2}{3} \text {. }
$$

entnommen werden, wenn darin $\mathrm{q}$ durch $\gamma$ (Gl. 33a) ersetzt wird. Bei ungerader Nutenzahl sind diese Wicklungsfaktoren noch mit dem Faktor $\cos \nu \frac{90^{\circ}}{\mathrm{N}}$ (Gl. $33 \mathrm{~b}$ ) zu multiplizieren, der sich in den praktisch wichtigsten Fällen $\left(\frac{N}{\nu} \geqq 10\right)$ aber nicht wesentlich von der Einheit unterscheidet.

\begin{tabular}{|c|c|c|c|c|c|c|c|}
\hline \multirow{2}{*}{$\ddot{q}$} & \multirow[b]{2}{*}{$Q$} & \multicolumn{6}{|c|}{$-\nu=$} \\
\hline & & I & 3 & 5 & 7 & 9 & II \\
\hline 2 & 3 & 0,866 & 0 & 0,866 & 0,866 & o & 0,866 \\
\hline 4 & 6 & $0,83^{6}$ & 0 & 0,224 & 0,224 & 0 & $0,83^{6}$ \\
\hline 6 & 9 & $0,83^{\mathrm{T}}$ & 0 & 0,188 & 0,154 & 0 & $0, I_{54}$ \\
\hline 8 & I2 & 0,829 . & 0 & $0,17^{8}$ & $0,13^{6}$ & o & 0,109 \\
\hline Io & I5 & 0,828 & 0 & o, I 73 & 0,129 & 0 & 0,095 \\
\hline 20 & 30 & 0,827 & 0 & 0,167 & $0, \mathrm{I} 2 \mathrm{I}$ & 0 & 0,079 \\
\hline$\infty$ & $\infty$ & 0,827 & 0 & 0,165 & 0,118 & o & 0,075 \\
\hline
\end{tabular}

\section{Tafel I3.}

Wicklungsfaktoren von einphasigen Ganzlochwicklungen mit $\frac{\mathrm{q}}{\mathrm{Q}}=\frac{2}{3}$ sowie von einphasigen Bruchlochwicklungen mit $\frac{2 \gamma}{N}=\frac{2}{3}$, wenn in der Tafel q durch $\gamma$ und $Q \operatorname{durch} \frac{N}{2}$ ersetzt werden.

Um die Größe der Wicklungsfaktoren der Bruchlochwicklungen auch bei

$$
2 \gamma \geqslant \frac{2}{3}
$$

beurteilen zu können, betrachten wir zunächst den Nenner in Gl. 33 a. In fast allen praktisch wichtigen Fällen $\left(\frac{N}{\nu} \geqq I 0\right)$ können wir den Sinus durch den Winkel ersetzen. Es ist dann der Nenner

$$
\gamma \sin \nu \frac{\mathrm{I} 8 \mathrm{o}^{\circ}}{\mathrm{N}} \approx \nu \frac{\gamma \pi}{\mathrm{N}}
$$


und wenn ungefähr $2 / 3$ sämtlicher Nuten bewickelt werden,

$$
\frac{\gamma}{\mathrm{N}} \approx \frac{\mathrm{I}}{3}
$$

wird der Nenner

$$
\gamma \sin \nu \frac{180^{\circ}}{\mathrm{N}} \approx 1,05 \nu \approx \nu
$$

Der Wicklungsfaktor ist also umgekehrt proportional der Ordnungszahl $\boldsymbol{\nu}$ der Welle; er ist immer kleiner als $\frac{I}{\nu}$, weil der Zähler in Gl. 33 a niemals größer als I ist. Durch passende Wahl von $\frac{\mathrm{N}}{\gamma}$ kann eine der Oberwellen noch ganz zum Verschwinden gebracht werden. Es muss dann

$$
\frac{\nu \gamma}{N}=\operatorname{gan} z
$$

sein, was z. B. für die 3., 5., 7., 9. oder II. Welle eintritt, wenn $\frac{N}{\gamma}=3,21 / 2,3 \%, 3 \quad$ " $23 / 4$ ist.

Bei den einphasigen Bruchlochwicklungen mit $\mathrm{N}$ und $\mathrm{p}$ teilerfremd lassen sich die resultierenden Oberwellen der E.M.K., die von der Nutung des Ankers hervorgerufen werden, bis zur n $\stackrel{\text { ten }}{=}$ Art (vgl. S. 234) nahezu vollkommen unterdrücken, wenn

$$
\frac{\gamma}{\mathrm{p}}=\operatorname{ganz}
$$

und bei gerader Nutenzahl N (Abb. 17a und 18a)

bei ungerader Nutenzahl $N(A b b ., 16 a)$

$$
\mathrm{n}<\mathrm{p} \text {, }
$$

$$
\mathrm{n} \overline{\overline{<}} \mathrm{p}
$$

ist. Dies folgt ohne weiteres daraus, daß für die Oberwellen $n \stackrel{\text { tex }}{=}$ Art die gegenseitige Phasenverschiebung der Einzelspannungen der Wicklung bei gerader Nutenzahl (vgl. Abb. I7 b und Abb. I8b)

bei ungerader Nutenzahl (vgl. Abb. I6b)

$$
\beta_{\mathrm{n}}=\mathrm{n} \frac{36 \mathrm{o}^{0}}{\mathrm{p}}
$$

$$
\beta_{\mathrm{n}}=\mathrm{n} \frac{360^{\circ}}{2 \mathrm{p}}
$$

ist.

Für die Wicklung in Abb. I7c (Nutenstern in Abb. I7 a) ist $\mathrm{N}=26=$ gerade, $\mathrm{p}=3$ und $\frac{\gamma}{\mathrm{p}}=3=$ ganz. Deshalb werden hier die Oberwellen .erster und zweiter Art nahezu vollkommen unterdrückt.

Die Oberwellen erster Art werden auch bei zweipoligen Wicklungen nahezu vollkommen unterdrückt, weil die Nutenzahl $N$ dieser Wicklungen immer ungerade ist.

Die Wicklungen, welche durch Multiplikation der Nuten- und Polpaarzahl einer Urwicklung ( $N$ und $p$ teilerfremd) mit demselben Faktor entstanden sind, haben dieselben Wicklungsfaktoren, und die von der Nutung des Ankers herrührenden Oberwellen werden in demselben Grade unterdrückt wie bei der Urwicklung.

9. Symmetrische dreiphasige Bruchlochwicklungen mit nur drei unbewickelten Nuten $(\eta=3)$; Nutenzahl (N) und Polpaarzahl (p) teiler: fremd $(\mathbf{t}=\mathbf{I})$. Da die Nutenzahl $N$ nach Gl. 30 immer ungerade ist, haben alle $2 \gamma$ Einzelspannungen im Strang verschiedene Phase. Sie lassen sich deshalb auch bei gerader Spulenzahl $\gamma$ nicht in Gruppen mit gleichen resultierenden Spannungen zer- 
legen. Alle Spulen eines Wicklungsstranges müssen also bei diesen Wicklungen in Reihe geschaltet werden.

Nach Gl. 30 muß die Nutenzahl $N$ auch durch 3 teilbar sein. Alle Polpaarzahlen, die den Teiler 3 haben $(p=3,6,9,12,15,18$ usw.) scheiden also bei der in diesem Abschnitt $z u$ behandelnden Wicklungen aus.

Ausführbare Polpaarzahlen sind:

I. die Polpaarzahl $\mathrm{I}$ und alle ganzen Potenzen von 2, also $\mathrm{p}=\mathrm{I}, 2,4,8$, I 6 usw. bei jeder beliebigen Spulenzahl $\gamma$ im Strang;

2. alle ungeraden, nicht durch 3 teilbaren Zahlen, also $\mathrm{p}=5,7$, I I, I3, I 7, I9 usw. bei solchen Spulenzahlen $\gamma$, für die $\frac{2 \gamma+\mathrm{I}}{\mathrm{p}}$ ) gebrochen ist;

3. alle geraden nicht durch 3 teilbaren Zahlen, die keine Potenzen von 2 sind, also $\mathrm{p}=\mathrm{IO}, \mathrm{I} 4,20 \mathrm{usw}$. bei solchen Spulenzahíen $\gamma$, für die $\left.\frac{2 \gamma+\mathrm{I}}{\mathrm{u}}{ }^{2}\right)$ gebrochen ist, wenn $u$ den größten ungeraden Teiler von $\mathrm{p}$ bezeichnet.

In Tafel I2 sind die Zahlen $Q$, deren zugehörige Nuten- und Polpaarzahlen Wicklungen dieses Abschnitts ermöglichen, ohne Klammern 'gedruckt. Sie sind nur für solche Polpaarzahlen ausführbar, für die auch dreiphasige Bruchlochwicklungen, deren sämtliche Nuten bewickelt sind, ausgeführt werden können. Man hat jedoch bei den Wicklungen dieses Abschnitts eine etwas größere Freiheit in der Wahl der Nuten- und Spulenzahl, auch lassen sich die Wicklungen bei richtiger Wahl der Spulenzahl immer so entwerfen, daß die durch die Nutung des Ankers hervorgerufenen Oberwellen fast vollständig unterdrückt werden.

An einem Ausführungsbeispiel für eine Wicklung mit

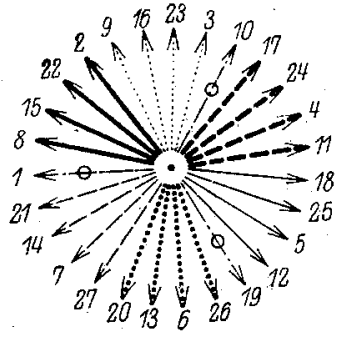

Abb. I9a. Nutenstern einer dreiphasigen Bruch. lochwicklung (Abschn. 9) mit $\mathrm{N}=27, \mathrm{p}=4, \gamma=4$, $\eta=3, Q=I^{1 / 8}, q=I$. $\mathrm{N}=27$ Nuten und $\mathrm{p}=4$ Polpaaren wollen wir den Entwurf der Wicklung" erläutern. Die Phase der fortlaufend numerierten Nuten ist in Abb. I9 a dargestellt. Damit die Wicklung symmetrisch wird, müssen die Phasen der drei unbewickelten Nuten um $120^{\circ}$ gegeneinander verschoben sein; es ist angenommen, daß die Nuten I, Io und I9, deren Spannungsstrahlen in Abb. I9 a durch strichpunktierte Linien mit kleinen Kreisen angedeutet sind, unbewickelt bleiben. Damit die Wicklung möglichst gut ausgenützt wird, müssen die $\gamma=4$ Strahlen der gleichsinnigen Spulenseiten desselben Wicklungsstrangs im Spannungsstern nebeneinander liegen, am besten - aber nicht notwendig. - so, daß die gleichsinnigen Strahlen desselben Wicklungsstrangs nicht durch den Strahl einer unbewickelten Nut unterbrochen werden. Es entsteht so der Nutenstern in Abb. I9 a und die Einzelspannungen der Wicklung in Abb. I9b; die Strahlen verschiedener Wicklungsstränge sind durch verschiedene Strichartè $(-,-\ldots-, \cdots)$, der verschiedene Sinn der Spulenseiten durch verschiedene Strichstärken unterschieden. In Abb. I9c ist der aus dem Nutenstern in der früher erläuterten Weise abgeleitete Schaltplan aufgezeichnet. Sämtliche Spulen müssen in Reihe geschaltet werden.

Für eine zweipolige Wicklung mit 9 Nuten stellt Abb. 20a den Nütenstern dar, aus dem sich die Einzelspannungen der Wicklung in Abb. $20 \mathrm{~b}$ und der Schaltplan in $20 \mathrm{c}$ ergeben.

1) Diese Bedingung für $\gamma$ stimmt mit unserer Voraussetzung, da $B \mathrm{~N}$ und $\mathrm{p}$ teilerfremd sein sollen, überein; denn es ist

$$
\mathrm{N}=3(2 \gamma+\mathrm{I})
$$

und der Teiler 3 kommt in der Polpaarzahl nicht vor.

$\left.{ }^{2}\right)$ Diese Bedingung für $\gamma$ stimmt mit unserer Voraussetzung, daß $N$ und $p$ teilerfremd sein sollen, überein, weil der Teiler 3 nicht in der Polpaarzahl vorkommt und $\mathrm{N}$ immer ungerade ist. 
Die Wicklungsfaktoren der dreiphasigen Bruchlochwicklungen dieses $\mathrm{Ab}$ schnitts werden nach Gl. 8 mit

$$
\begin{aligned}
\mathrm{s}=2 \gamma, \varphi & =\frac{360^{\circ}}{2 \mathrm{~N}} \text { und } \mathrm{N}=6 \gamma+3: \\
\xi_{\nu} & =\frac{\sin \nu \frac{2 \gamma}{2 \gamma+\mathrm{I}} 30^{\circ}}{2 \gamma \sin \nu \frac{30^{\circ}}{2 \gamma+\mathrm{I}}} .
\end{aligned}
$$

und sind in Tafel I 4 für $\nu$ bis I I und verschiedene Werte von $\gamma$ zusammengestellt. In den meisten Fällen sind hiernach die Wicklungsfaktoren der Wellen I., 3., 5. und

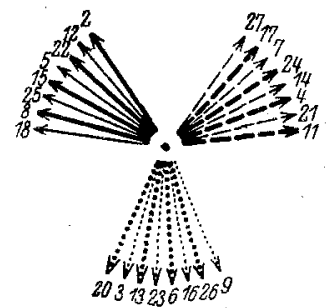

Abb. I9b. Einzelspannungen fur die Wicklung zu Abb. 19a.

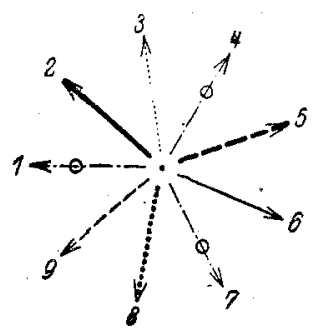

Abb. 2oa. Nutenstern einer dreiphasigen Bruchlochwicklung (Abschn. 9) mit $N=9, p=I$, $y=\mathrm{I}, \quad \eta=3, \quad \mathrm{Q}=\mathrm{I}^{1 / 2}, \mathrm{q}=\mathrm{I}$.

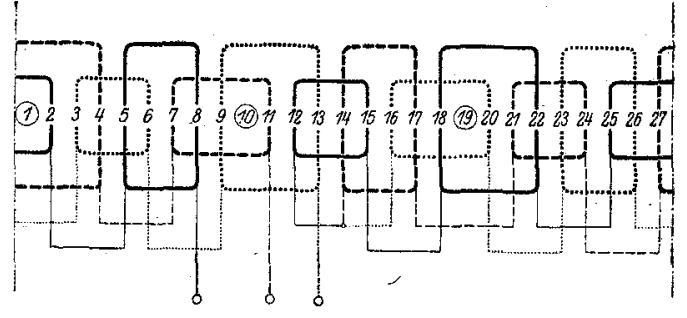

Abb. Igc. Schaltplan einer dreiphasigen Bruchlochwicklung nach $A b b$. Iga und $b$. Reihenschaltung in den Strängen; Sternschaltung der Stränge.

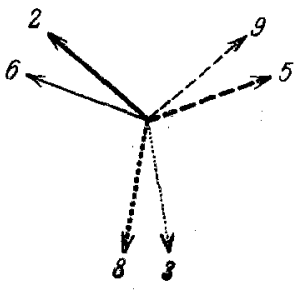

Abb. 20 b. Einzelspannungen für die Wicklung zu Abb. 20 a.

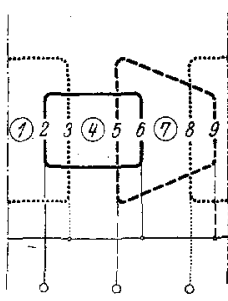

Abb. 20c. Schaltplan einer dreiphasigen Bruchloch wicklung nach Abb. zoa und b. Sternschaltung der Stränge (Spulen)

I I. Ordnung größer, die Oberwellen 7 . und 9. Ordnung dagegen kleiner als bei den Bruchlochwicklungen nach Abschnitt 4 mit derselben Spulenzahl $\gamma$ im Strang (vgl. Tafel I).

$$
\text { Tafel I4. }
$$

\begin{tabular}{|c|c|c|c|c|c|c|}
\hline \multirow[b]{2}{*}{$\gamma$} & \multicolumn{6}{|c|}{$v=$} \\
\hline & I & 3 & 5 & 7 . & 9 & II \\
\hline $\mathbf{I}$ & 0,985 & 0,866 & 0,643 & $0,34^{2}$ & 0 & $0,34^{2}$ \\
\hline 2 & 0,973 & 0,769 & 0,433 & 0,078 & 0,182 & 0,272 \\
\hline 3 & 0,968 & $0,73^{\circ}$ & 0,357 & $o$ & 0,209 & 0,222 \\
\hline 4 & 0,965 & 0,709 & 0,317 & 0,037 & 0,216 & 0,192 \\
\hline 6 & 0,962 & 0,688 & 0,277 & 0,072 & 0,220 & 0,160 \\
\hline IO & 0,959 & 0,667 & 0,243 & 0,098 & o,219 & o.I33 \\
\hline 20 & 0,957 & $0,65^{2}$ & $0,2 \mathrm{r} 7$ & $0, x I 9$ & 0,216 & $0, \mathrm{II} \mathrm{I}$ \\
\hline$\infty$ & 0,955 & 0,637 & O,I9I & 0,136 & 0,212 & 0,088 \\
\hline
\end{tabular}

Wicklungsfaktoron der dreiphasigen Bruchlochwicklungen mit 3 unbewickelten Nuten und $t=I$ ( $\mathrm{N}$ und $\mathrm{p}$ teilerfremd, Abschnitt 9 ). 
Für die Unterdrückung der von der Nutung des Ankers herrührenden Oberwellen der E.M.K. gelten dieselben Überlegungen wie bei der Einphasenwicklung (vgl. S. 253). Bei zweipoligen Wicklungen werden die Oberwellen erster Art, bei den übrigen Wicklungen die Oberwellen bis zur $\mathrm{n} \stackrel{\text { ten }}{=}$ Art nahezu vollkommen unterdrückt, wenn

und

$$
\frac{\gamma}{\mathrm{p}}=\operatorname{gan} z
$$

$$
\mathrm{n} \equiv \mathrm{p}
$$

ist. Diesen Bedingungen genügt z. B. unser Ausführungsbeispiel nach $A b b .19 a, b$ und $c$ für Oberwellen bis zur 4. Art.

I0. Die übrigen symmetrischen dreiphasigen Bruchlochwicklungen mit nur drei unbewickelten Nuten $(\boldsymbol{\eta}=\mathbf{3})$. Da wir die Wicklungen, deren Nutenzahl $N$ und Polpaarzahl $p$ teilerfremd sind, bereits behandelt haben, muß bei den Wicklungen dieses Abschnitts $t$ immer größer als I sein. Nach Gl. $30 \mathrm{c}$ muß dann zwischen der Spulenzahl $\gamma$ im Strang und dem Teiler $t$ die Beziehung

$$
\gamma=g t+\frac{t-I}{2}
$$

bestehen; woraus zunächst folgt, daß der Teiler $t$ ungerade sein muß. Deshalb scheiden hier alle Polpaarzahlen aus, die eine Potenz von 2 sind (also $\mathrm{p}=2,4,8$, 16 ụsw.). Für alle übrigen Polpaarzahlen lassen sich symmetrische Bruchlochwicklungen ausführen, also für die Polpaarzahlen $p=3,5,6,7,9$, IO, I I, I2, I3, I4, I5, I7, I8, I9, $20 \mathrm{usw}$. Besonders wichtig sind die Wicklungen für die sehr häufig vorkommende Polpaarzahl $\mathrm{p}=3$, für die sich, wie wir gesehen haben, Bruchlochwicklungen, deren sämtliche Nuten bewickelt sind, nicht ausführen lassen.

Die Zahl der ausführbaren Spulenzahl $\gamma$ im Wicklungsstrang wird nach Gl. 3oc durch den größten Teiler $t$ bestimmit, den $N$ und $\mathrm{p}$ gemeinsam haben. Diese Zahlen $\gamma$ sind in Tafel 15 für $\mathrm{t}$ bis 19 zusammengestellt. In Tafel $\mathrm{I} 2$ sind die Zahlen $Q$, die den zugeordneten Nuten- und Polpaarzahlen der Wicklungen dieses Abschnitts entsprechen, in eckige Klammern gesetzt. Trotzdem bej den Wicklungen $t>I$ ist, sind, wie wir nach Behandlung der Ausführungsbeispielé sehen werden, parallele Wicklungszweige nicht möglich.

\section{Tafel I5.}

\begin{tabular}{|c|c|c|c|c|c|c|c|c|c|}
\hline $\mathrm{t}=$ & 3 & 5 & 7 & 9 & II & I3 & I5 & I7 & I9 \\
\hline & I & 2 & 3 & 4 & 5 & 6 & 7 & 8 & 9 \\
\hline & 4 & 7 & 10 & I3 & 16 & I9 & 22 & 25 & 28 \\
\hline & 7 & 12 & $\mathrm{I} 7$ & 22 & 27 & $3^{2}$ & 37 & $4^{2}$ & 47 \\
\hline & 10 & ${ }^{27}$ & 24 & $3^{x}$ & $3^{8}$ & 45 & $5^{2}$ & 59 & 66 \\
\hline & I3 & 22 & $3^{I}$ & 40 & 49 & $5^{8}$ & 67 & -76 & 85 \\
\hline & I6 & 27 & $3^{8}$ & 49 & 60 & $7^{I}$ & 82 & 93 & $\mathrm{TO}_{4}$ \\
\hline & - & - & - & - & - & - & - & - & - \\
\hline
\end{tabular}

Spulenzahlen $\gamma$ im Strang, die für Bruchlochwicklungen mit 3 unbewickelten Nuten und $t>1$ ausführbar sind.

Wir wollen den Entwurf der Wicklung an einem Beispiel für $\mathrm{N}=27$ Nuten und $\mathrm{p}=3$ Polpaare erläutern. Das Verhältnis von Nutenzahl und Produkt aus Polpaarzahl und Strangzahl ist dann 
die Spulenzahl im Strang

$$
Q=\frac{27}{2 \cdot 3 \cdot 3}=1^{1 / 2}
$$

$$
\gamma=\frac{N-\eta}{6}=4
$$

und die Nutenzahl für Pol und Strang

Der gemeinsame Teiler $t$ von $\mathrm{N}$ und $\mathrm{p}$ ist 3 , je drei Nuten haben also dieselbe Phase. Wir erhalten den Spannungsstern in'Abb. 2 I a, worin die Einzelstrahlen zunächst die Phasen der fortlaufend numerierten Nuten angeben. Benachbarte Strahlen entsprechen hier auch benachbarten Nuten, da $\mathrm{t}=\mathrm{p}$ ist (vgl. Gl. 6 und $6^{\prime}$ ).

Die drei unbewickelten Nuten müssen in der Phase um $120^{\circ}$ verschoben sein. Lassen wir z. B. die Nut I frei, dann müssen außerdem noch die Nuten 4 oder I 3 oder 22 und 7 oder 16 oder 25 unbewickelt bleiben. Welche dieser Ausführungen die zweckmäßigste ist, kann nach Aufzeichnung des SchaItplans festgestellt werden. Wir wollen zunächst annehmen, daß die Nuten I, 4 und 7 frei bleiben.

Jetzt können wir die übrigen Nuten auf die drei Wicklungsstränge verteilen: Dabei werden wir uns wieder davon leiten lassen, daß die Resultierende der Einzelspannungen eines Wicklungsstranges möglichst groß sein soll. Gleichphasige Nuten müssen nun bei den Wicklungen dieses $\mathrm{Ab}$ schnittes zum Teil verschiedenen Wicklungssträngen angehören. Man überzeugt sich leicht, daß die Resul tierende der Einzelspannungen eines Wicklungsstranges

$$
\mathrm{q}=\frac{\gamma}{3}=\mathrm{I}^{1} / 3 .
$$

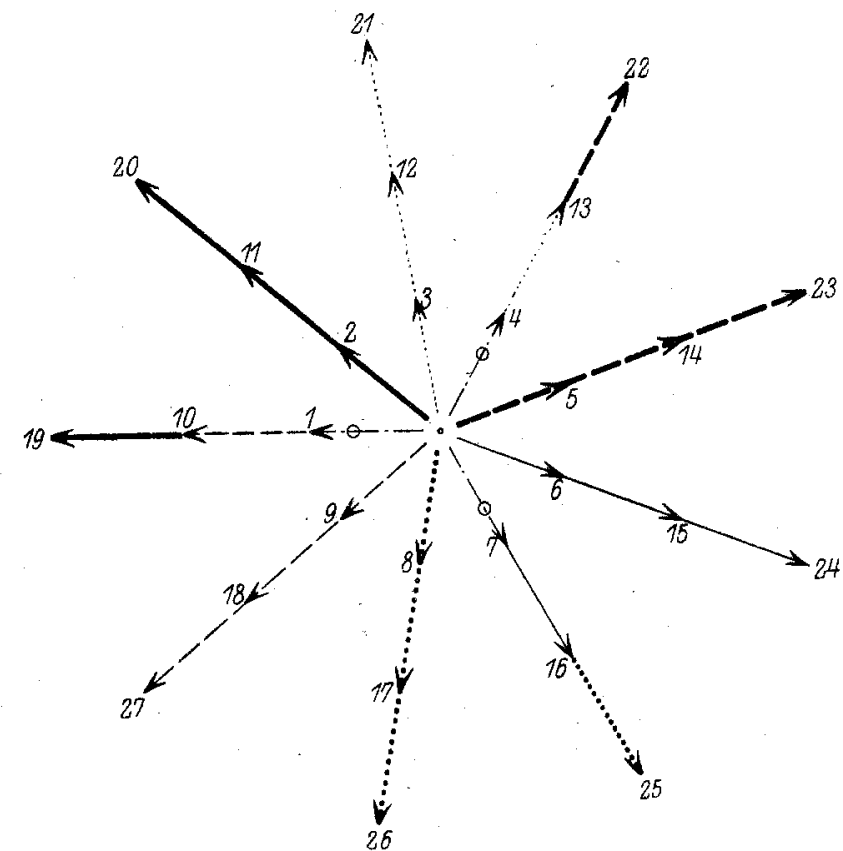

Abb. 2. a. Nutenstern einer dreiphasigen Bruchlochwicklung (Abschn. Io) mit $\mathrm{N}=27, \mathrm{p}=3, \gamma=4, \eta=3, Q=\mathrm{I}^{1 / 2}, \mathrm{q}=\mathrm{I}^{1 / 3}$. (Günstigste Ausnutzung der Wicklung).

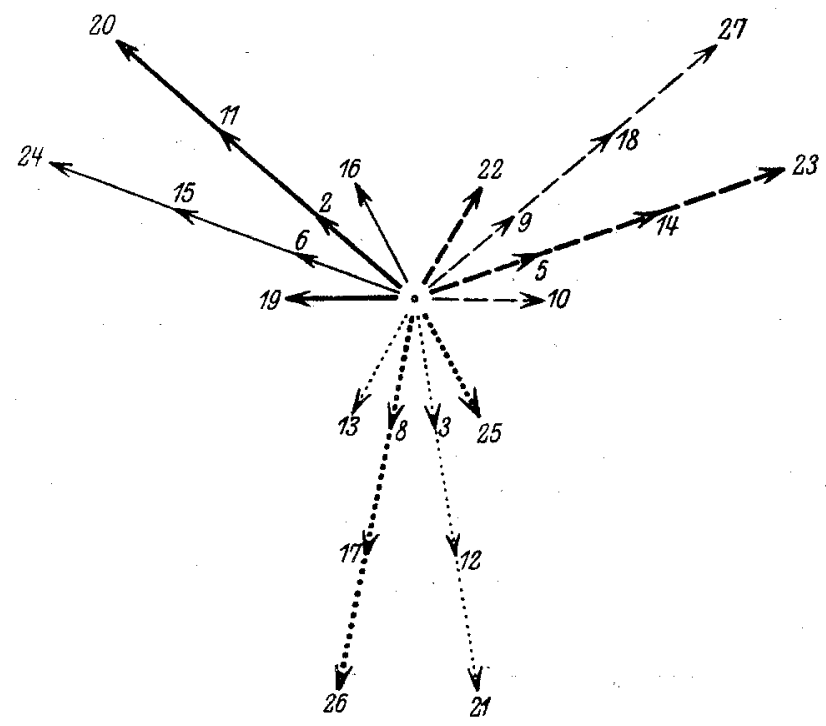

Abb. 2rb. Einzelspannungen für die Wicklung zu Abb. 2т a. 
am größten wird, wenn die gleichphasigen Nuten, die verschiedenen Wicklungssträngen angehören, auch mit einer unbewickelten Nut phasengleich sind, wie es für

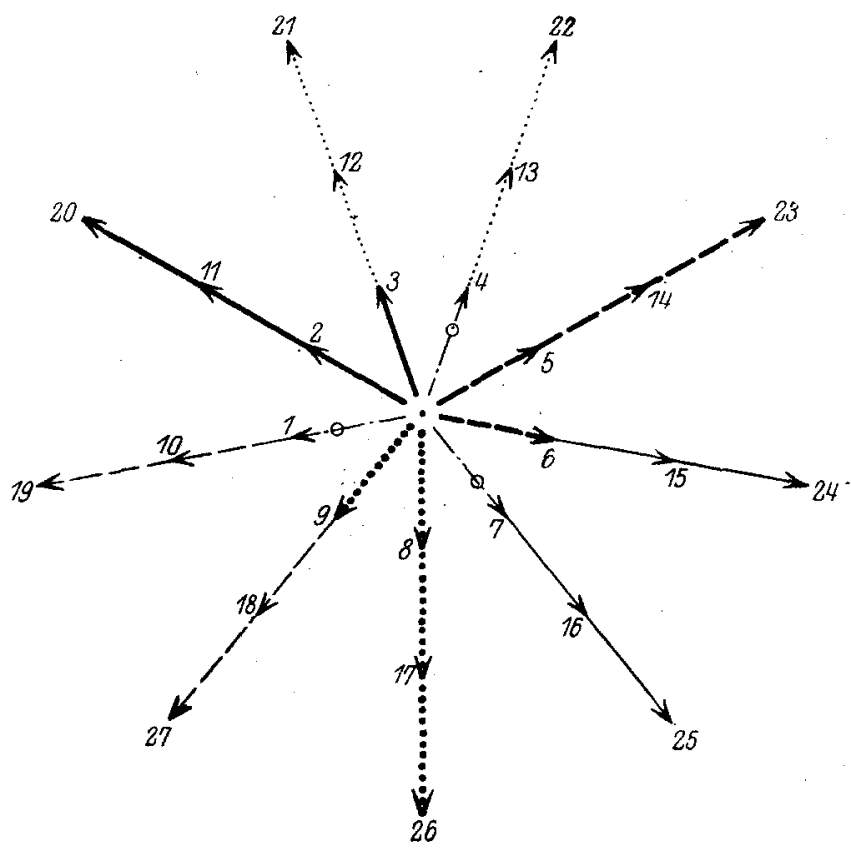

Abb. 22a. Nutenstern einer dreiphasigen Bruchlochwicklung (Abschnitt тo) mit derselben Nutenund Polpaarzahl wie Abb. 2I a, aber ungünstigerer Ausnutzung der Wicklung.

unser Ausführungsbeispiel in Abb. 2 I a angenommen ist. Sind die gleichphasigen Nuten, die verschiedenen Wicklungssträngen angehören, nicht auch mit einer unbewickelten Nut phasengleich, wie z. B. in Abb. $22 \mathrm{a}$, so ist die Resultierende der

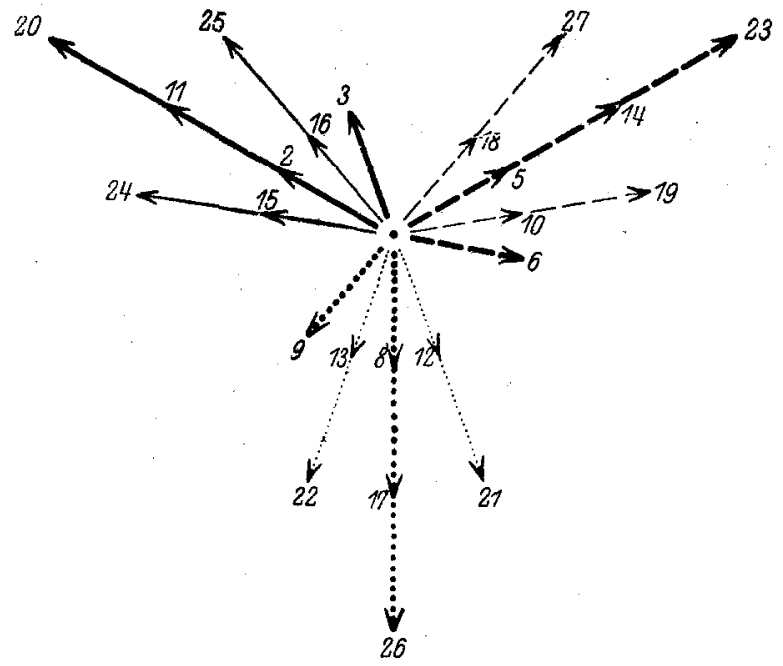

Abb. 22 b. Einzelspannungen für die Wicklung $z u$ Abb. 22 a. Einzelspannungen im Strang kleiner, wie man durch Vergleich der Abb. $2 \mathrm{Ib}$ und $22 \mathrm{~b}$ erkennt, die die Einzelspannungen im Wicklungsstrang darstellen.

Die Aufteilung der einzelnen Nuten auf dio einzelnen Wicklungsstränge erfolgt am schnellsten, indem man von

$$
\gamma=g t+\frac{t-I}{2}
$$

gleichsinnigen Spulenseiten (Gl. 30 c) $\frac{t-I}{2}$ Spulenseiten in Nuten legt, die mit einer unbewickelten Nut phasengleich sind, die übrigen gt Spulenseiten lassen sich dann in $\mathrm{g}$ im Nutenstern unmittelbar nebeneinander liegende Gruppen von je $t$ gleichphasigen Nuten unterbringen (vgl. auch Abb. 23 a mit $\gamma=7, \mathrm{~g}=2$ und $\frac{\mathrm{t}-\mathrm{I}}{2}=\mathrm{I}$ ). Dadurch ist dann auch die Lage der übrigen Spulenseiten bestimmt. 
In Abb. $2 \mathrm{I}$ a ist $\gamma=4, \mathrm{~g}=\mathrm{I}$ und $\frac{\mathrm{t}-\mathrm{I}}{2}=\mathrm{I}$. Es liegen die positiven Spulenseiten des ersten Wicklungsstrangs $(\longrightarrow)$ in den

Nuten I9, 2, I I, 20, die negativen Spulenseiten des dritten Wicklungsstrangs $(\ldots \ldots)$ in den

Nuten 3, I2, 2 I, I3, die positiven Spulenseiten des zweiten Wicklungsstrangs (- - ) in den

Nuten 22, 5, 14, 23, die negativen Spulenseiten des ersten Wicklungsstrangs (- - ) in den

Nuten $6,15,24,16$, die positiven Spulenseiten des dritten Wicklungsstrangs ( $\cdots \cdots)$ in den

Nuten $25,8,17,26$, die negativen Spulenseiten des zweiten Wicklungsstrangs $(--\infty)$ in den Nuten 9, I8, 27, IO. Die Nuten 1, 4, 7 (-..-.- ) bleiben unbewickelt.

Aus Abb. 2 I a erhalten wir wieder den Schaltplan der Wicklung, indem wir je eine positive mit einer negativen Spulenseite desselben Wicklungsstrangs zu

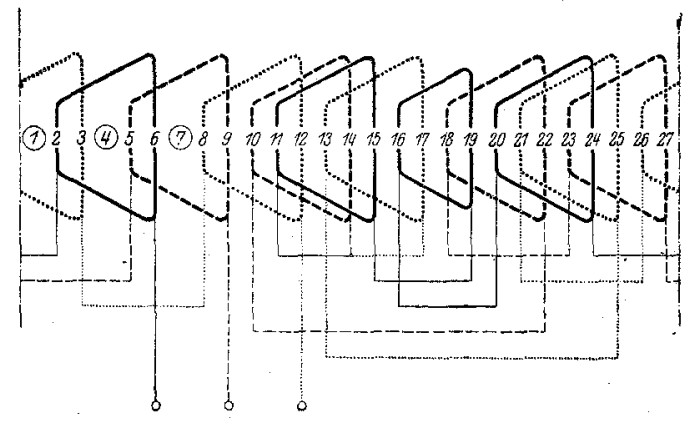

Abb. 2I c. Schaltplan einer dreiphasigen Bruchlochwicklung nach Abb. 2i a und $b$. Sternschaltung.

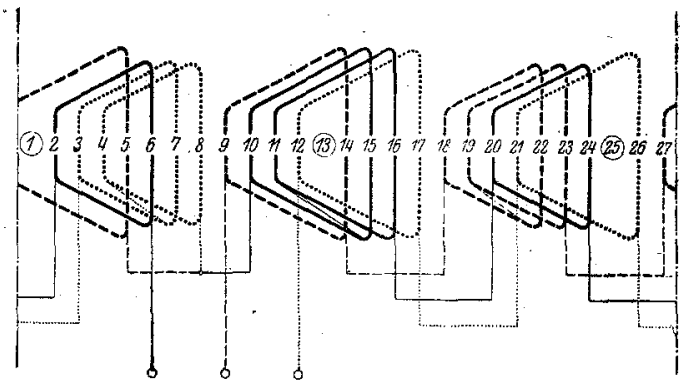

Abb. 2I d. Schaltplan einer aus Abb. 21 a abgeleiteten dreiphasigen Bruchlochwicklung mit teilbarem Anker. Sternschaltung.

Spulen vereinigen, und die Schaltenden so legen, daß immer ungleichsinnige Spulenseiten miteinander verbunden werden. Eine Wicklung mit Gruppen von gleichachsigen Spulen, der sogenannten Spulenordnung in zwei Ebenen, ist bei der Nutenaufteilung nach Abb. 2 r a nicht möglich, es werden deshalb zweckmäßig gekröpfte Spulen, wie, im Schaltplan Abb. 2 I $c$ angedeutet, verwendet, die alle im wesentlichen dieselbe Form erhalten. Elf Spulen haben hier dieselbe Weite, nämlich gleich 4 Nutteilungen, während die Weite einer Spule (I6-I9) um eine Nutteilung verkürzt ist.

Einfacher wird die Wicklung, wenn im Nutenstern der Abb. 2 I a die Spulenseiten in den gleichphasigen Nuten Io und 19 vertauscht werden und die Spulenseite der Nut 13 in die gleichphasige unbewickelte Nut 4, die

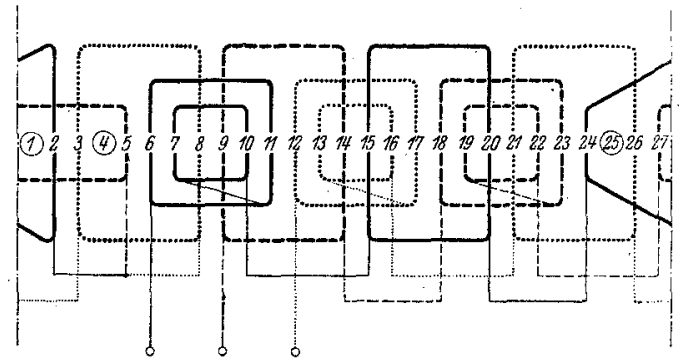

Abb. 2i e. Schaltplan einer aus Abb. aI a abgeleiteten dreiphasigen Bruchlochwicklung mit Wicklungsköpfen in zwei Ebenen. Sternschaltung. der Nut 25 in die gleichphasige unbewickelte Nut 7 gelegt wird. Dieser Nutenaufteilung entspricht der Schaltplan in Abb. 2Id. Wir erhalten hier je sechs Spulen mit einer Weite von 4 und 5 Nutteilungen. Die Schaltverbindungen werden hier wesentlich einfacher als in Abb. $21 \mathrm{c}$ 
und der Anker kann an drei um $1 / 3$ des Ankerumfangs auseinander liegende Stellen (zwischen den Nuteri 26 und 27, 8 und 9, I 7 und I 8 ) geteilt werden, ohne daß Spulenquerverbindungen von der Teilungsebene geschnitten werden.

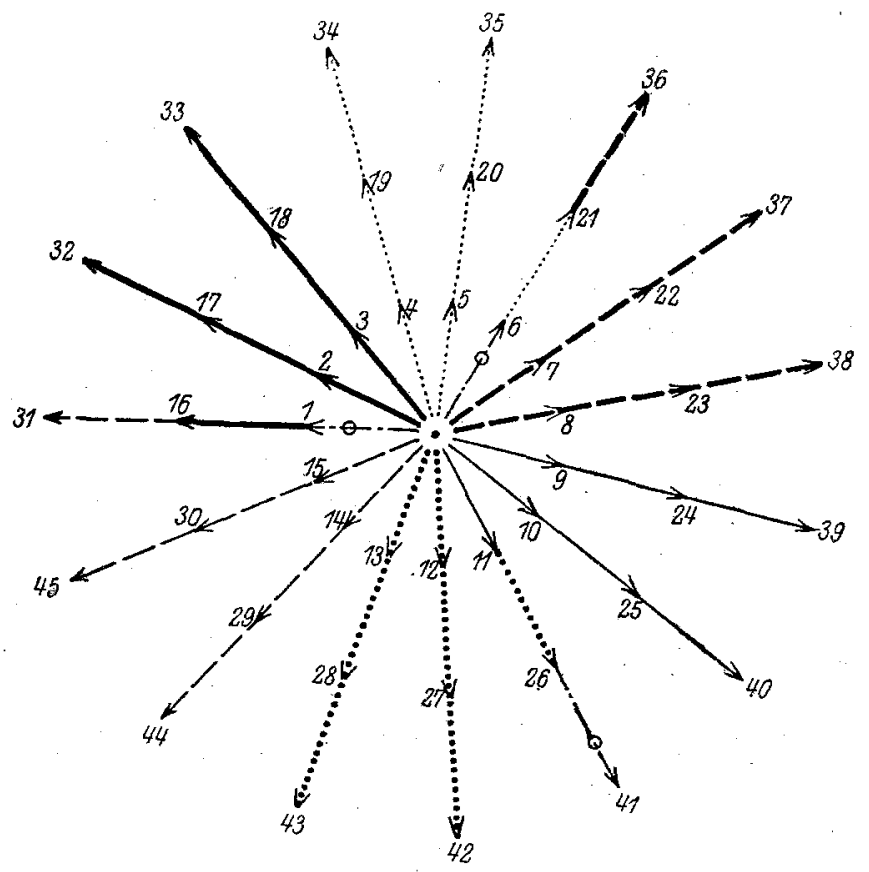

Abb. 23a. Nutenstern einer dreiphasigen Bruchlochwicklung (Abschn, Io) mit $N=45, \mathrm{p}=3, y=7, \eta=3, Q=2^{1} / 2, q=2^{1} / 3$.

Die Spulenköpfe lassen sich auch in zwei Ebenen anordnen, wenn wir in Abb. 2 I a die Spulenseiten in den gleichphasigen Nuten ro und I9 vertauschen, die

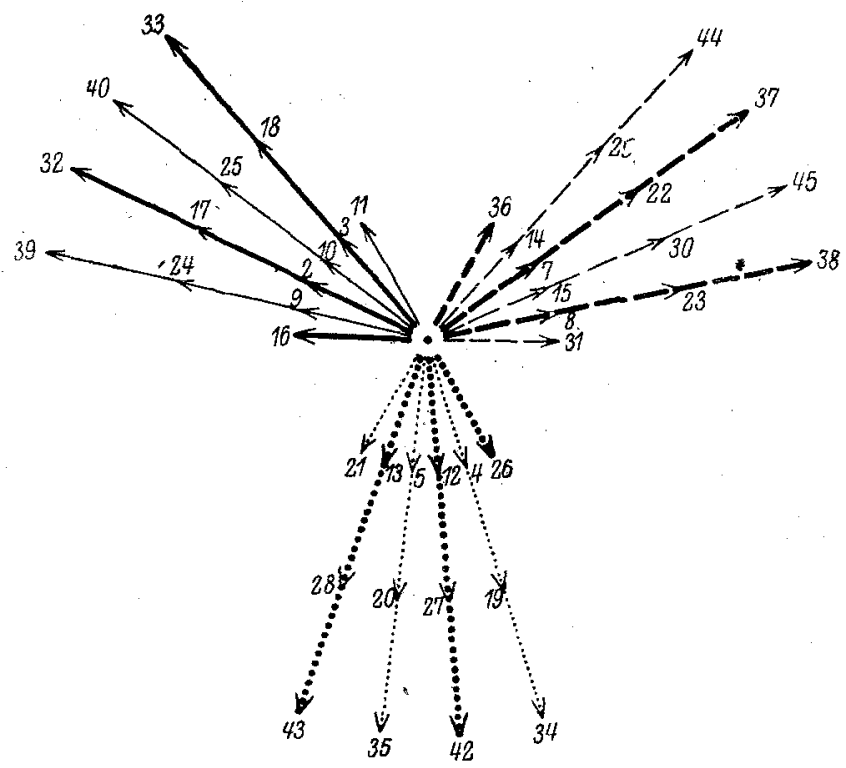

Abb. 23b. Einzelspannungen für die Wicklung zu Abb. $23 \mathrm{a}$.

Spulenseite der Nut 16 in die unbewickelte gleichphasige Nut 7 und die Spulenseite der Nut 25 in die frei gewordene gleichphasige Nut 16 legen. Der Schaltplan 
für diese Nutenaufteilung ist in Abb. 2 I e dargestellt. Ein Wicklungskopf (von Nint 24 nach 2) muß hier, wie auch bei jeder sechspoligen Ganzlochwickiung, von der einen Ebene in die andere ubergeführt werden.

Um die günstigste Aufteilung der verschiedenen Wicklungssträngen angehörigen gleichphasigen Nuten auf die einzelnen Wicklungsstränge zu finden, bedarf es einiger Übung. Am schnellsten kommt mas zum Ziel, wenn man die Spulenseiten, die nicht mehr willkürlich verlegt werden körnen, im Schaltplan aufeichnet, und dann den Einfluß der Lage der übrigen Spulenseiten auf den Schaltplan untersucht.

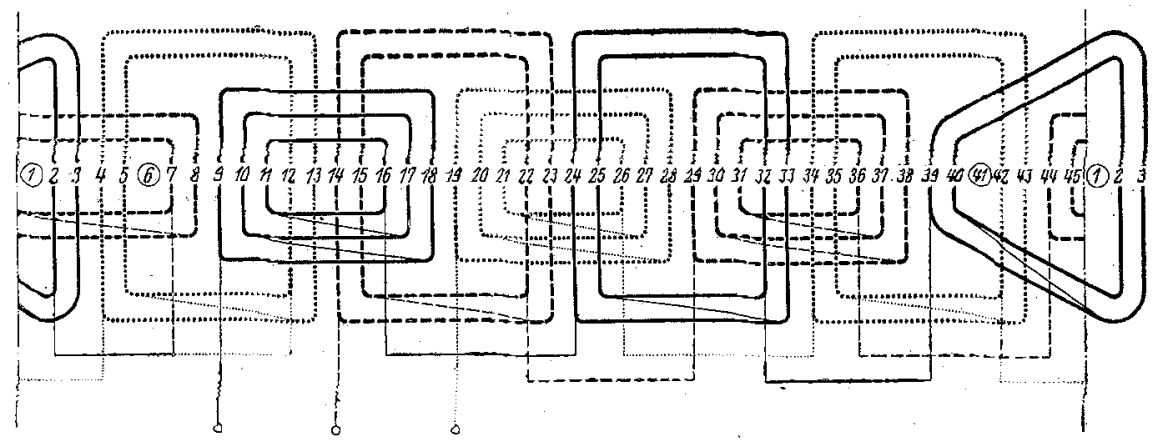

Abb. 23c. Schaltplan einer dreiphasigen Bruchlochwicklung nach Abb. 23a und b mit Wick lungsköpfen in zwei Ebenen. Sternschaltung.

In Abb. $23 \mathrm{a}$ ist noch der Nutenstern einer Wicklung für dieselbe Polpaarzahl $(p=3)$ aber $\gamma=7$ Spulen in Strang aufgezeichnet, aus dem der Schaltplan in Abb. $23 \mathrm{c}$ abgeleitet ist. Die Einzelspannungen im Strang werden durch Abb. $23 \mathrm{~b}$ dargestellt.

Für alle sechspoligen Wicklungen dieses Abschnitts läßt sich die Form und die Zahl der Einzelspulen eines Wicklungskopfes durch Abb. 24 darstellen.

Bei Wicklungen mit andern Polpaarzahlen aber demselben gemeinsamen Teiler von Nutenzahl $N$ und Polpaarzahl p unter: scheidet sich der Nutenstern nur durch die Numerierung der Strahlen. Nebeneinander liegende Nuten sind dann immer um $\frac{\mathrm{P}}{\mathrm{t}}$ nebeneinander liegende Strahlen im

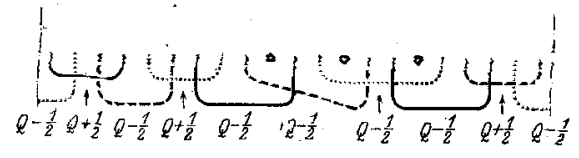

Abb. 24. Form und Zahl der Spulenquer. verbindungen bei den sechspoligen Bruch. lochwicklungen des Abschnitts ro. Spannungsstern verschoben (vgl. Gi. 6 und $6^{\prime}$ ).

Bei allen Wicklungen dieses Abschnitts lassen sich die Einzelspannungen im Strang in $2 \mathrm{~g}$ Gruppen von je $\mathrm{t}$ gleichphasigen Strahlen und zwei Gruppen von je $\frac{t-I}{2}$ gleichphasigen Strahlen zusammenfassen (vgl. z. B. Abb. 25 mit $g=3, t=7$ und $\left.\frac{t-1}{2}=3\right)$. Sämtliche $2(g+1)$ Strahlengruppen sind, wie man leicht übersieht, um den Phasenwinkel

$$
\alpha^{\prime \prime}=\frac{\mathrm{t}}{2 \mathrm{~N}} 360^{\circ}
$$

gegeneinasder verschoben; die beiden Strahlengruppen von je $\frac{t-1}{2}$ Strablen schließen den größten Phasenwinkel ein. Die Einzelspannungen mit solchen Phasen lassen sich nicht in Gruppen zerlegen, deren Resultierende gleiche Größe und Phase haben. Auch bei den Wicklungen dieses Abschnitts müssen deshalb alle Spulen eines Wicklungsstrangs in Reihe geschaltet werden. 
Zur Bestimmung des Wicklungsfaktors der in diesem Abschnitt behandelten Bruchlochwicklungen vereinigen wir die $2 \gamma$ Einzelspannungen im Wicklungsstrang (vgl. Gl. 30c und Abb. 25) zu zwei Gruppen. Die eine Gruppe (Abb. 25a) enthält die $2 \mathrm{gt}$ Einzelspannungen, von denen immer $\mathrm{t}$ phasengleich sind, und die andere Gruppe (Abb. 25b) die übrigen $t$ - I Einzelspannungen. Der Wicklungsfaktor der ersten Gruppe ist dann nach Gl. 8 mit

$$
\begin{gathered}
\mathrm{s}=2 \mathrm{~g} \text { und } \varphi=\frac{360^{\circ}}{2 \mathrm{~N}} \mathrm{t}=\frac{60^{\circ}}{2 \mathrm{~g}+\mathrm{I}} \\
\xi_{\nu}^{\prime}=\frac{\sin \nu \frac{2 \mathrm{~g}}{2 \mathrm{~g}+\mathrm{I}} 30^{\circ}}{2 \mathrm{~g} \sin \nu \frac{30^{\circ}}{2 \mathrm{~g}+\mathrm{I}}}
\end{gathered}
$$

er ist für die Grundwelle immer etwas größer äls der einer Ganzlochwicklung mit $\mathrm{q}=2 \mathrm{~g}$. Für die zweite Strahlengruppe ist

$$
s=2 \text { und } \varphi=\nu 60^{\circ}
$$

der Wicklungsfaktor dieser Gruppe also

$$
\xi_{\nu}^{\prime \prime}=\frac{\sin \nu 60^{\circ}}{2 \sin \nu 30^{\circ}}
$$

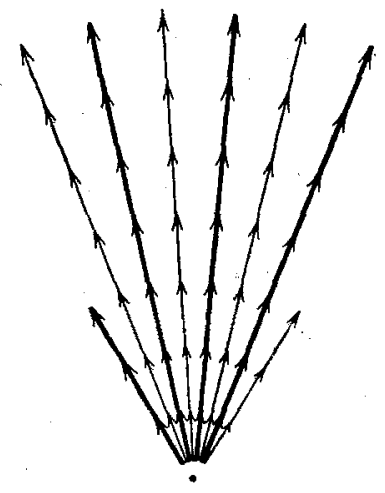

Abb. 25. Einzelspannungen im Strang einer Bruchlochwicklung mit drei unbewickelten Nuten (Abschn. IO) und $\gamma=24, \mathrm{~g}=3$,

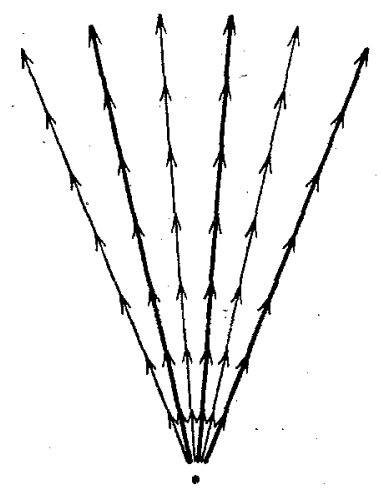

Abb. 25a. Erste Gruppe der Einzelspannungen in Abb. 25.

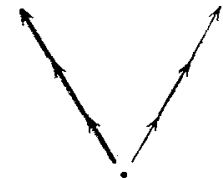

Abb. 25 b. Zweite Gruppe der Einzelspannungen in Abb. 25.

$$
\mathfrak{t}=7, \frac{\mathrm{t}-\mathrm{I}}{\mathrm{z}}=3 \text {. }
$$

er ist für die Grundwelle wesentlich kleiner als bei den dreiphasigen Ganzlochwicklungen. Dadurch wird der resultierende Wicklungsfaktor des ganzen Wicklungsstranges für die Grundwelle und für kleine Werte von $g$ etwas kleiner als der einer Ganzlochwicklung mit $q=2 \mathrm{~g}$. Die Resultierenden der beiden Strahlengruppen sind immer phasengleich; deshalb erhalten wir den resultierenden Wicklungsfaktor zu

$$
\xi_{\nu}=\frac{\mathrm{gt} \xi_{\nu}^{\prime}+\frac{\mathrm{t}-\mathrm{I}^{*}}{2} \xi^{\prime \prime}{ }_{\nu}}{\gamma}
$$

Die Faktoren $\xi^{\prime}{ }_{v}$ können der Tafel 14 entnommen werden, wenn wir darin $\gamma$ durch $g$ ersetzen. Die Faktoren $\xi^{\prime \prime}{ }_{\nu}$ sind für solche Werte von $\nu$, die durch 3 teilbar sind, null, für alle übrigen ungeradzahligen $\nu$ ist ihr absoluter Betrag $\frac{1}{2} \sqrt{3}=0,866$. In den Tafeln $I 6 a, b ; c$ und $d$ sind die resultierenden Wicklungsfaktoren nach Gl. 37 für $t=3,5,7$ und 9 zusammengestellt. 
Tafel 16a.

Wicklungsfaktoren der dreiphasigen Bruchlochwicklungen mit 3 unbewickelten Nuten und $t>I$ (Abschnitt ro).

$$
\mathrm{t}=3
$$

\begin{tabular}{|c|c|c|c|c|c|c|c|}
\hline \multirow{2}{*}{$\mathrm{g}$} & \multirow{2}{*}{$\gamma$} & \multicolumn{6}{|c|}{$\nu=$} \\
\hline & & I & 3 & 5 & 7 & 9 & II \\
\hline 0 & I & 0,866 & 0 & 0,866 & 0,866 & 0 & 0,866 \\
\hline I & 4 & 0,955 & 0,636 & 0,699 & 0,473 & 0 & 0,473 \\
\hline 2 & 7 & 0,943 & 0,669 & 0,495 & 0,190 & 0,279 & 0,357 \\
\hline 3 & 10 & $0,95^{8}$ & 0,657 & 0,108 & $0,0 \varepsilon_{7}$ & 0,275 & 0,286 \\
\hline 4 & I3 & 0,957 & 0,654 & 0,359 & 0,100 & 0,120 & 0,244 \\
\hline 6 & I9 & 0.957 & $0,65^{2}$ & 0,308 & $0, I_{3}$ & 0,207 & o,I97 \\
\hline 10 & $3^{I}$ & 0,956 & 0,646 & 0,263 & 0,123 & $0,24^{\circ}$ & o, I 56 \\
\hline 20 & 61 & $0,95^{6}$ & 0,642 & 0,228 & $0,3^{I}$ & 0,227 & 0,123 \\
\hline$\infty$ & $\infty$ & 0,955 & 0,637 & O,IgI & 0,136 & 0,212 & 0,088 \\
\hline
\end{tabular}

Tafel $16 \mathrm{~b}$.

Wicklungsfaktoren der dreiphasigen Bruchlochwicklungen mit 3 unbewickelten Nuten und $t>$ I (Abschnitt ro).

$$
\mathrm{t}=\mathbf{5}
$$

\begin{tabular}{|c|c|c|c|c|c|c|c|}
\hline \multirow{2}{*}{$\mathrm{g}$} & \multirow{2}{*}{$\gamma$} & \multicolumn{6}{|c|}{$v=$} \\
\hline & & I & .3 & 5 & .7 & 9 & II \\
\hline 0 & 2 & 0,866 & 0 & 0,866 & 0,866 & 0 & 0,866 \\
\hline I & 7 & $0,95 \mathrm{I}$ & 0,6r9 & 0,707 & 0,492 & o & 0,492 \\
\hline 2 & 12 & 0,955 & 0,624 & 0,505 & 0,209 & $0,15^{\circ}$ & 0,379 \\
\hline 3 & I7 & $0,95^{6}$ & 0,644 & 0,417 & 0,102 & 0,184 & 0,297 \\
\hline 4 & 22 & 0,955 & 0,644 & 0,367 & $0, \mathrm{II} 2$ & 0,197 & 0,253 \\
\hline 6 & 32 & $0,95^{6}$ & 0,645 & 0,314 & $0, \mathbf{1 2 I}$ & 0,206 & 0,204 \\
\hline Io & $5^{2}$ & $0,9.55$ & 0,665 & 0,267 & 0,128 & 0,211 & $0,16 \mathrm{I}$ \\
\hline 20 & 102 & 0,954 & 0,640 & 0,230 & 0,124 & 0,212 & 0,125 \\
\hline$\infty$ & $\infty$ & 0,955 & 0,637 & O,IgI & $0,13^{6}$ & 0,212 & 0,088 \\
\hline
\end{tabular}


Tafel I6c.

Wicklungsfaktoren der dreiphasigen Bruchlochwicklungen mit 3 unbewickelten Nuten und $t>I$ (Abschnitt Io).

$$
\mathrm{t}=7
$$

\begin{tabular}{|c|c|c|c|c|c|c|c|}
\hline \multirow[b]{2}{*}{$\mathbf{g}$} & \multirow[b]{2}{*}{$\gamma$} & \multicolumn{6}{|c|}{$\nu=$} \\
\hline & & I & 3 & 5 & 7 & 9 & II \\
\hline 0 & 3 & 0,866 & 0 & 0,866 & 0,866 & 0 & 0,866 \\
\hline I & ro & 0,949 & 0,606 & 0,710 & 0,499 & 0 & 0,499 \\
\hline 2 & I 7 & 0,954 & 0,634 & 0,509 & 0,217 & $0,15^{\circ}$ & 0,377 \\
\hline 3 & 24 & 0,955 & $0,63^{8}$ & 0,420 & 0, I08 & 0,183 & 0,302 \\
\hline 4. & $3^{I}$ & 0,955 & 0,640 & 0,370 & 0,117 & 0,195 & 0,257 \\
\hline 6 & 45 & 0,955 & 0,642 & 0,316 & $0,12_{4}$ & 0,205 & 0,207 \\
\hline Io & 73 & 0,955 & 0,640 & 0,268 & 0,130 & 0,210 & 0,163 \\
\hline 20 & I 43 & 0,955 & 0,639 & 0,273 & 0,134 & 0,212 & 0,126 \\
\hline$\infty$ & $\infty$ & 0,955 & 0,637 & $0, \mathrm{I} 9 \mathrm{I}$ & 0,136 & 0,212 & 0,088 \\
\hline
\end{tabular}

Tafel I6d.

Wicklungsfaktoren der dreiphasigen Bruchlochwicklungen mit 3 unbewickelten Nuten und $t>I$ (Abschnitt ro).

$$
\mathrm{t}=9
$$

\begin{tabular}{|c|c|c|c|c|c|c|c|}
\hline \multirow[b]{2}{*}{$\mathrm{g}$} & \multirow[b]{2}{*}{$\gamma$} & \multicolumn{6}{|c|}{$y=$} \\
\hline & & I & 3 & 5 & 7 & 9 & II \\
\hline 0 & 4 & 0,866 & o & . 0,866 & 0,866 & 0 & 0,866 \\
\hline $\mathbf{I}$ & ${ }_{3}$ & $0,94^{8}$ & 0,599 & 0,711 & 0,503 & 0 & 0,503 \\
\hline 2 & 22 & 0,953 & 0,629 & $0,5^{12}$ & 0,221 & $0, I_{4} 8$ & $0,3^{80}$ \\
\hline 3 & $3^{\mathrm{I}}$ & 0,955 & 0,636 & 0,422 & 0,112 & 0,182 & 0,305 \\
\hline 4 & 40 & 0,955 & 0,638 & $0,37^{2}$ & 0,120 & 0,195 & 0,259 \\
\hline 6 & $5^{8}$ & 0,955 & 0,640 & $0,3^{18}$ & 0,126 & 0,205 & 0,209 \\
\hline Io & 94 & 0,955 & 0,639 & 0,269 & $0,3^{I}$ & 0,210 & 0,164 \\
\hline 20 & 184 & 0,955 & 0,638 & $0,23 \mathrm{I}$ & 0,135 & 0,2 II & 0,127 \\
\hline$\infty$ & $\infty$ & 0,955 & 0,637 & O,I9I & 0,136 & 0,212 & 0,088 \\
\hline
\end{tabular}


Die von der Nutung des Ankers herrührenden Oberwellen erster Art werden nahezu vollkommen unterdrückt, wenn die Nutenzahl durch die Polpaarzahl teilbar ist, wenn also

$$
\frac{\mathrm{N}}{\mathrm{p}}=\text { gan } z,
$$

ist. Dann entsprechen, wie wir gesehen haben, benachbarte Nuten auch benachbarten Strahlen im Spannungsstern. Da N nun immer ungerade ist, sind im Stern der Einzelspannungen des Wicklungsstrangs immer die Spannungen einer positiven und negativen Spulenseite in der Phase um $1 / 2$ Nutteilung verschoben, heben sich also für die Oberwellen erster Art im wesentlichen auf.

In allen übrigen Fällen $(t<p)$ werden die Oberwellen der Nutung nur unvollkommen unterdrückt. In jedem Einzelfalle läßt sich der Grad der Unterdrückung an Hand der Gl. $30 \mathrm{c}$ und des Spannungssterns in Abb. 25 leicht feststellen. Besondere Beachtung verdienen noch die Wicklungen, bei denen $g=\frac{p}{t}$ oder ein Vielfaches davon ist. Die Oberwellen von 2 gt Einzelstrahlen werden dann bis zur $\mathrm{n}=\mathrm{g} \stackrel{\text { ten }}{=}$ Art nahezu vollkommen, der Rest von $\mathrm{t}-\mathrm{I}$ Strahlen nur unvollkommen unterdrückt, so daß in der Resultierenden des Wicklungsstrangs von der Amplitude jeder Oberwelle der Nutung weniger als

wirksam ist.

$$
v=\frac{t-I}{2 g t+t-I}
$$

II. Symmetrische dreiphasige Bruchlochwicklungen mit mehr als drei unbewickelten Nuten $(\eta>3)$. Aus den in Abschnitt 9 und ro behandelten und in Tafel 12 zusammengestellten Bruchlochwicklungen lassen sich neue Bruchlochwicklungen mit demselben Verhältnis $Q$ von Nutenzahl und Produkt aus Polzahl und Strangzahl ableiten, indem Nutenzahl N und Polpaarzahl p der Urwicklung mit demselben Faktor $t^{\prime}$ multipliziert werden, so daß die Nutenzahl der neuen Wicklung $\mathfrak{t}^{\prime} \mathrm{N}$ und die Polpaarzahl $\mathrm{t}^{\prime} \mathrm{p}$ ist. Die Spulen eines Wicklungsstrangs lassen sich dann zu $\mathfrak{t}^{\prime}$ gleichwertigen Gruppen vereinigen, die nach Belieben parallel oder in Reihe geschaltet werden können. Die Zahl der unbewickelten Nuten ist hierbei $3 t^{\prime}$. In Tafel I2 sind diese praktisch weniger wichtigen Bruchlochwicklungen nicht vermerkt, da sie auch für Nutenzahlen ausführbar sind, die nicht am Kopf der Tafel I2 stehen und Tafel I2 dadurch unübersichtlich werden würde.

Da symmetrische dreiphasige Bruchlochwicklungen mit drei unbewickelten Nuten auch für die Polpaarzahl $\mathrm{p}=\mathrm{I}$ ausführbar sind, ist es wie bei einer Ganzlochwicklung stets möglich, eine Bruchlochwicklung mit $\mathrm{p}$ gleichwertigen Spulengruppen auszuführen. Die Zahl der unbewickelten Nuten ist dann immer $3 \mathrm{p}$. Je nachdem eine solche Wicklung mit $Q=\mathrm{I}^{1 / 2}, 2^{1 / 2}, 3^{1 / 2}$ usw. ausgeführt wird, sind von sämtlichen Ankernuten nur 2/3, 4/5, $6 / 2$ usw. bewickelt. Der Ankerumfang der Bruchlochwicklung mit $\mathrm{t}^{\prime}=\mathrm{p}$ parallelen Wicklungszweigen ist also besonders bei kleinen Werten von $Q$ schlecht ausgenützt.

Aus der im Abschnitt 9 (S. 255, Abb. 20c) behandelten Wicklung mit $\mathrm{N}=9$ und $\mathrm{p}=\mathrm{I}$ erhalten wir $\mathrm{z}$. B. eine Wicklung mit $\mathrm{N}=3 \cdot 9=27$ und $\mathrm{p}=3 \cdot \mathrm{I}=3$, indem wir den Schaltplan der Abb. $20 \mathrm{c}$ dreimal wiederholen. Es entsteht so der Schaltplan in Abb. 26a, der sich noch so abändern läßt (vgl. Abb. 26b), daß die Querverbindungen von nur einer Spule (gekröpfte Spule) in zwei verschiedenen Ebenen liegen. Die drei gleichwertigen Wicklungszweige sind in den Abbildungen parallel geschaltet.

Vergleichen wir die Wicklung in Abb. 26b mit der Bruchlochwicklung in Abb. 2I e, die bei nur drei unbewickelten Nuten für dieselbe Nuten und Polpaarzahl gilt, so erkennen wir, daß sie durch Weglassen der Spulen 7-IO, I3-I6 und 
19-22 in Abb. 2 I e und durch Verlegung der Spulenquerverbindungen entsteht. Die Möglichkeit der Parallelschaltung von Wicklungszweigen wird also durch eine unvollkommenere Ausnützung des Ankerumfangs erkauft. Der Wicklungsfaktor der Grundwelle ist bei der Wicklung Abb. 26b etwas größer $(0,985)$ als der nach Abb. 2 I e $(0,955)$. Die von der Nutung des Ankers herrührenden Oberwellen der E.M.K. erster Art werden -bei beiden Schaltungen fast vollkommen unterdrückt.

Alle Wicklungen dieses Abschnitts, die aus den Wicklungen in den Abschnitten 9 und Io durch Multiplikation der Nutenzahl und der Polpaarzahl mit démselben Faktor $\left(t^{\prime}\right)$ entstehen, haben auch denselben Wicklungsfaktor wie die Wicklungen, aus denen sie abgeleitet sind, und die Oberwellen der E.M.K., die von der Nutung des Ankers herrühren, werden in demselben Grade unterdrückt.

Die übrigen symmetrischen Bruchlochwicklungen mit mehr als drei freien Nuten entstehen aus den Ganzlochwicklungen und den Bruchlochwicklungen mit unbewickelter Nutenzahl, indem in jedem Strang einige Spulen wegbleiben, und beanspruchen deshalb wohl keine weitere Beachtung.

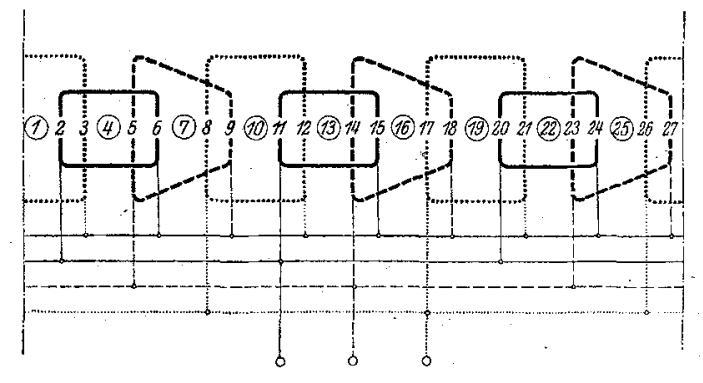

Abb. 26a. Schaltplan einer dreiphasigen Bruchlochwicklung (Abschn. II) mit drei parallelen Wicklungszweigen und drei gekröpften Spulen. $\mathrm{N}=\mathbf{2 7}$, $\mathrm{P}=3, \gamma=3, \eta=6, Q=\mathrm{I}^{1} / 2, \quad \mathrm{q}=\mathrm{I}$. Vgl. Abb. 2r e.

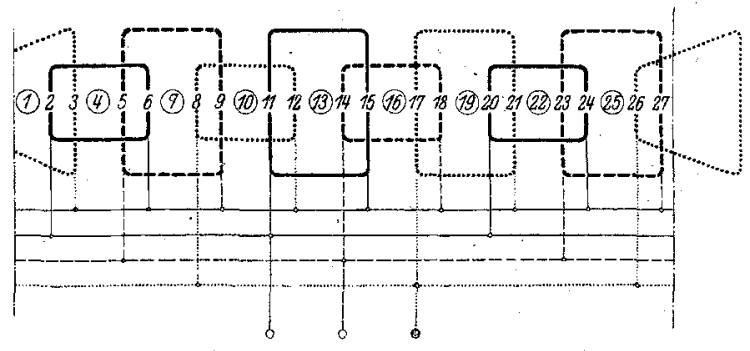

Abb. 26b. Schaltplan einer dreiphasigen Bruchlochwicklung (Abschn. II) mit drei parallelen Wicklungszweigen und einer gekröpften Spule. $\mathrm{N}=27, \mathrm{p}=3, \gamma=3$, $\eta=6, Q=\mathrm{I}^{1 / 2}, \mathrm{q}=\mathbf{1}$.

Vgl. Abb. 2r e.

12. Übersicht der behandelten Bruchlochwicklungen. Alle Bruchlochwicklungen lassen sich auf gewisse Wicklungen, die Urwicklüngen, zurückführen mit denselben elektrischen Eigenschaften. Aus jeder Urwicklung läßt sich eine neue Bruchlochwicklung ableiten, indem die Nutenzahl $\mathrm{N}$ und die Polpaarzahl $\mathrm{p}$ der Urwicklung mit demselben Faktor $\left(t^{\prime}\right)$ multipliziert werden. Die Wicklungsfaktoren der abgeleiteten Wicklungen sind ebenso groß, und die von der Nutung des Ankers herrührenden Oberwellen werden in demselben Maße unterdrückt wie bei der entsprechenden Urwicklung. Die mögliche Zahl der parallelen Wicklungszweige ist aber bei einer abgeleiteten Wicklung $t^{\prime}$ mal so groß wie bei der Urwicklung. Die Bruchlochwicklungen mit mehreren unbewickelten Nuten lassen sich auch für die Polpaarzahl $\mathrm{p}=\mathrm{I}$ ausführen.

Der Entwurf der Bruchlochwicklungen ergibt sich aus dem Nutenstern in der in den einzelnen Abschnitten, besonders Abschnitt 4 und 9 erläuterten Weise. Bei den Wicklungen, deren Nutenzahl N und Polparahl p teilerfremd sind, kann die Wicklung unmittelbar mit dem Nutenschritt $r$ nach Gl. I 4 aufgezeichnet werden.

Es werden in der Arbeit nur die symmetrischen Wicklungen behandelt, die bei derselben Nuten- und Polpaarzahl den größten. Wicklungsfaktor der Grundwelle haben. Die andern Wicklungen sind schlechter ausgenützt, doch können sie zuweilen kleinere Oberwellen der Spannung ergeben. Diese Wicklungen müssen in jedem besonderen Falle aus dem Spannungsstern abgeleitet werden. 
Die symmetrischen Bruchlochwicklungen, bei denen alle Nuten bewickelt sind, sind in den Tafeln 5 bis 7 zusammengestellt. Am Kopfe der Tafel sind die Spulenzahl $\gamma$ im Strang und die Nutenzahl N, an der linken Seite der Tafel die Polpaarzahl $\mathrm{p}$ angegeben, wobei die vom Verband Deutscher Elektrotechniker empfohlenen Polpaarzahlen ohne Klammern gedruckt sind. Die abgeleiteten Wicklungen sind durch Einklammerung der Nutenzahl q für Pol und Strang von den übrigen unterschieden. Jede abgeleitete Wicklung hat dieselbe Nutenzahl q für Pol und Strang wie die entsprechende Urwicklung. Diese läßt sich für jede abgeleitete Wicklung aus den Tafeln ablesen, da die abgeleitete Wicklung die. sclbe Nutenzahl q für Pol und Strang hat wie ihre Urwicklung. Die mögliche Zahl der parallelen Wicklungszweige kann den Tafeln entnommen werden. Sie bestimmt sich aus dem Verhältnis t; von Polpaarzahl $p$ und Nenner $n$ des Bruches $q$ und ist

$$
\mathrm{t}^{\prime}=\frac{\mathrm{p}}{\mathrm{n}} \operatorname{oder} 2 \mathrm{t}^{\prime}=2 \frac{\mathrm{p}}{\mathrm{n}},
$$

je nachdem das Verhältnis zwischen Spulenzahl $\gamma$ im Strang und $\mathfrak{t}^{\prime}$ gerade oder ungerade ist (vgl. S. 243).

Die Urwicklungen lassen sich in zwei Gruppen einteilen. Die erste Gruppe (Abschnitt 4) enthält alle zweiphasigen Urwicklungen und die dreiphasigen Urwicklungen, bei denen Nutenzahl $\mathrm{N}$ und Polpaarzahl $\mathrm{p}$ keinen gemeinsamen Teiler haben. Wenn die Spulenzahl $\gamma$ im Strang gerade ist, sind zwei parallele Wicklungszweige möglich, sonst müssen immer alle Spulen im Strang in Reihe geschaltet werden. Die Wicklungsfaktoren dieser Wicklungsgruppe sind dieselben wie bei einer Ganzlochwicklung, deren Nutenzahl q für Pol und Strang gleich der Spulenzahl $\gamma$ im Strang der Bruchlochwicklung ist (Tafel I). Die Oberwellen der Spannung, die von der Nutung des Ankers herrühren, lassen sich mehr oder weniger vollkommen unterdrücken; sie werden bei den Wicklungen mit

$$
\mathrm{q}=\mathrm{g} \mp \frac{\mathrm{I}}{\mathrm{p}}
$$

am vollkommensten unterdrückt, und zwar auf $\frac{\mathbf{I}}{\gamma}$ der Oberwellenamplituden einer entsprechenden Ganzlochwicklung.

Die zweite Gruppe der Urwicklungen (Abschnitt 5) umfaßt die dreiphasigen Bruchlochwicklungen (Tafel 7, q ohne Klammern), deren Nutenzahl $\mathrm{N}$ und Polpaarzahl p den Teiler 2 haben. Parallele Wicklungszweige sind hier nicht möglich. Die Wicklungsfaktoren der Oberwellen (Tafel II) sind wesentlich kleiner als bei der ersten Gruppe, so daß sich die Form der Spannungskurve wesentlich mehr der Sinusform nähert. Bei allen vierpoligen Wicklungen dieser Gruppe werden die von der Nutung des Ankers herrührenden Oberwellen erster Art nahezu vollkommen unterdrückt: Bei den übrigen Polpaarzahlen werden die Oberwellen erster und zweiter Art mehr oder weniger vollkommen unterdrückt, und zwar für die Wicklungen mit

$$
q=g \mp \frac{I}{p}
$$

am vollkommensten, auf etwas mehr als $\frac{\mathbf{I}}{\gamma}$ der Oberwellen einer entsprechenden Ganzlochwicklung.

Einphasige Bruchlochwicklungen mit mehreren unbewickelten Nuten lassen sich für jede Nutenzahl ausführen. Bei den hier behandelten Urwicklungen sind Nutenzahl $\mathrm{N}$ und Polpaarzahl $\mathrm{p}$ teilerfremd. Es sind zwei parallele Wicklungszweige möglich, wenn sowohl die Nutenzahl $\mathrm{N}$ als auch die Spulenzahl $\gamma$ gerade ist. Bei gerader Nutenzahl $N$ ist der Wicklungsfaktor derselbe wie bei einer Ganzlochwicklung (Tafel I3), deren Nutenzahl q für Pol und Strang 
gleich der Spulenzahl $\%$ und deren Verhältnis $Q$ aus gesamter Nutenzahl und Polzahl gleich $\frac{\mathrm{N}}{2}$ ist. Bei ungerader Nutenzahl $\mathrm{N}$ sind die Wicklungsfaktoren der Oberwellen etwas kleiner (Gl. $33 \mathrm{~b}$ ). Die von der Nutung des Ankers herrührenden Oberwellen erster Art (bei $\mathrm{p}>\mathrm{I}$ auch die zweiter Art) werden nahezu vollkommen unterdrückt, wenn

ist.

$$
\frac{\gamma}{\mathrm{p}}=\operatorname{gan} z
$$

Die Urwicklungen der symmetrischen dreiphasigen Bruchlochwicklungen mit nurdrei unbewickelten Nuten sind in Tafel 2 zusammengestellt. Am Kopfe der Tafel ist die Spulenzahl $\gamma$ im Strang und die gesamte Nutenzahl $\mathrm{N}$ angeschrieben, an der linken Seite der Tafel die Polpaarzahl p. Die zugehörigen Werte von Nutenzahl $\mathrm{N}$ und Polpaarzahl $\mathrm{p}$ sind für die Urwicklung durch das in der Tafel eingeschriebene Verhältnis $Q$ von Nutenzahl N und Produkt aus Polzahl $2 \mathrm{p}$ und Strangzahl $\mathrm{m}$ bezeichnet; die abgeleiteten Wicklungen sind in der Tafel nicht mit aufgenommen. Parallele Wicklungszweige sind bei den Urwicklungen nicht möglich.

Die dreiphasigen Urwicklungen lassen sich in zwei Gruppen einteilen. Bei der ersten Gruppe (Abschnitt 9) sind Nutenzahl $N$ und Polpaarzahl $p$ teilerfremd. Für diese Wicklungen ist die Verhältniszahl $Q$ ohne Klammern gedruckt. Die Wicklungsfaktoren der Wicklungen dieser Gruppe (Tafel I4) sind für die Oberwellen teilweise größer, teilweise kleiner als bei den Bruchlochwicklungen, deren Nuten alle bewickelt sind. Die von der Nutung des Ankers herrührenden Oberwellen erster Art (bei $\mathrm{p}>\mathbf{I}$ auch die zweiter Art) lassen sich nahezu vollkommen unterdrücken, wenn

ist.

$$
\frac{\gamma}{\mathrm{p}}=\operatorname{ganz}
$$

Die zweite Gruppe der Urwicklungen (Abschnitt Io) ist in Tafel I2 durch die eingeklammerten Verhältniszahlen $Q$ bezeichnet. Diese Wicklungen sind auch für die Polpaarzahl $\mathrm{p}=3$ ausführbar, wofür sich eine Bruchlochwicklung, deren sämtliche Nuten bewickelt sind, nicht ausführen läßt. Die Wicklungsfaktoren sind in den Tafeln $16 \mathrm{a}$ bis d zusammengestellt. Die von der Nutung des Ankers herrührenden Oberwellen erster Art lassen sich praktisch vollkommen unterdrücken, wenn

$$
\frac{\mathrm{N}}{\mathrm{p}}=\operatorname{ganz}
$$

ist. In allen übrigen Fällen werden die Oberwellen der Nutung unvollkommen uniterdrückt. 